\title{
Integration of Aeroservoelastic Properties into the NASA Dryden F/A-18 Simulator Using Flight Data from the Active Aeroelastic Wing Program
}

\author{
A Thesis \\ Presented to \\ The Faculty of \\ California Polytechnic State University, San Luis Obispo \\ In Partial Fulfillment \\ Of the Requirements for the Degree \\ Master of Science in Aerospace Engineering
}

By

Alexander Wong Chin

March 2011 
(C) 2011

Alexander Wong Chin

ALL RIGHTS RESERVED 


\section{Approval}

TITLE:

INTEGRATION OF AEROSERVOELASTIC PROPERTIES

INTO THE NASA DRYDEN F/A-18 SIMULATOR USING

FLIGHT DATA FROM THE ACTIVE AEROELASTIC WING

PROGRAM

AUTHOR: $\quad$ Alexander Wong Chin

DATE SUBMITTED: $\quad$ March, 2011

Dr. Eric Mehiel

Committee Chair

Associate Professor, Cal Poly Aerospace Engineering Department

Marty Brenner

Committee Member and Advisor

Aerospace Engineer, NASA Dryden Flight Research Center

Dr. Daniel Biezad

Committee Member

Professor, Cal Poly Aerospace Engineering Department

Dr. Colleen Kirk

Committee Member

Associate Professor, Cal Poly Mathematics Department 


\section{Abstract}

Integration of Aeroservoelastic Properties into the NASA Dryden F/A-18 Simulator Using Flight Data from the Active Aeroelastic Wing Program

\section{Alexander Wong Chin}

Aircraft structures have varying stiffness levels making them flexible. Consequently, this elastic property becomes increasingly important at high speeds affecting the flight dynamics of the aircraft. In high speed aircraft such as the F/A-18, elastic structural properties must be accounted for to ensure confidence in predicted flight dynamics in order to avoid adverse aeroelastic phenomena throughout flight.

Data from the F/A-18 Active Aeroelastic Wing (AAW) program was used to create aeroservoelastic (ASE) models at varying flight conditions. The discretized ASE models were integrated into the NASA Dryden F/A-18 simulator in parallel with the traditional 6-DOF (degrees-of-freedom) flight dynamics calculations to ensure minimal disruption to the existing operating framework of the simulator. An interpolation scheme was used to construct ASE models within the known flight condition models. Data was processed through the state-space ASE models to compute the elastic effects during flight. Total flight dynamics from the simulation were analyzed and showed expected behavior for the combined elastic and rigid-body components in flight. 


\title{
Acknowledgments
}

\author{
Marty Brenner, NASA Dryden Flight Research Center: \\ For mentoring me throughout my thesis and providing guidance in the world of \\ aeroservoelasticity, simulation, and controls. It has been an honor to work under your guidance \\ introducing me to the diverse world of structural dynamics in aircraft.
}

\section{Marlin Pickett, NASA Dryden Flight Research Center:}

For always being happy to help in my questions about the NASA Dryden F/A-18 simulator and working with UNIX and FORTRAN. Thank you for taking the time out of many of your lunch hours to help me in the simulation integration process.

\section{Ashante Jordan, NASA Dryden Flight Research Center:}

For being my initial 'go to' person for all of my questions about the F/A-18 simulator and programming in FORTRAN. 


\section{NASA Dryden Co-Workers:}

For being some of the best people anyone can work with. I had so many great conversations with many of you. Thank you for your advice and guidance throughout my time at NASA Dryden.

\section{Dr. Colleen Kirk, Cal Poly Mathematics Department:}

For being an excellent math teacher and teaching me so much of the applied mathematics I have used during my time with NASA and will undoubtedly use throughout my career.

\section{Dr. Eric Mehiel, Cal Poly Aerospace Engineering Department:}

For stepping up to be my committee chair under short notice and for your insights in teaching the graduate controls classes I have taken with you.

\section{Dr. Daniel Biezad, Cal Poly Aerospace Engineering Department:}

For joining my defense committee under short notice and teaching me the fundamental 'A,B,C's' of controls in my undergraduate controls classes.

\section{My Family:}

For always being there to guide and support me throughout my life. 


\section{Contents}

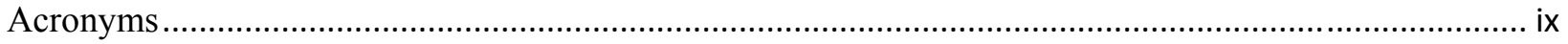

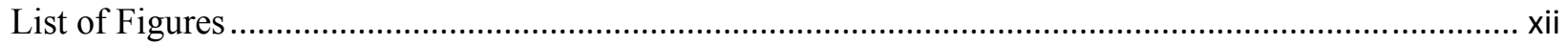

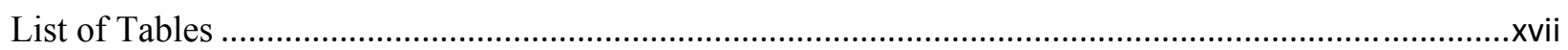

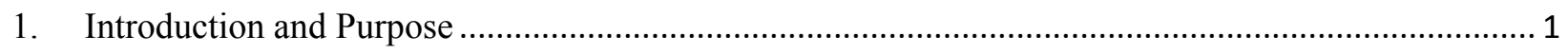

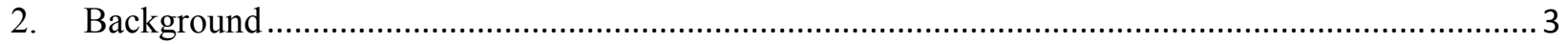

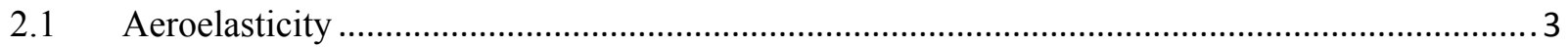

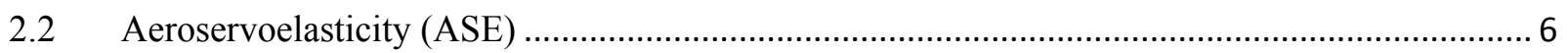

2.3 Active Aeroelastic Wing (AAW) Program …..................................................................... 8

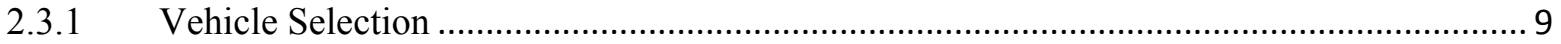

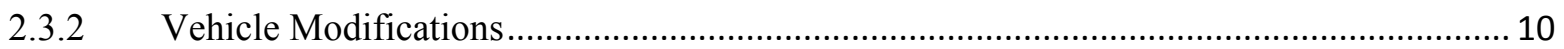

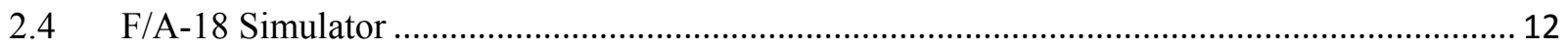

2.4.1 F/A-18 Simulator Background ...................................................................................... 12

2.4.2 Simulator Calculation Process …….............................................................................. 13

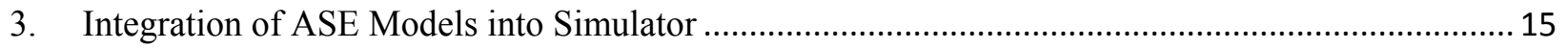

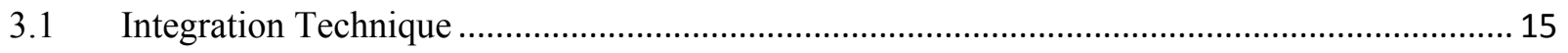

3.2 Using Data from AAW to Provide Elastic Characteristics to F/A-18 Simulator .......................20

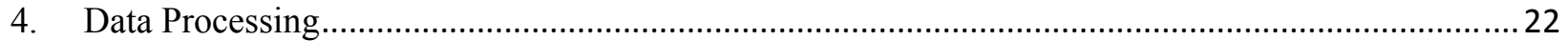

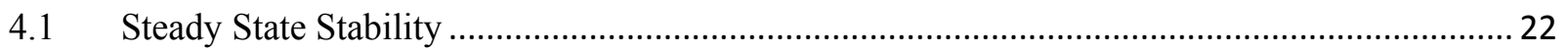

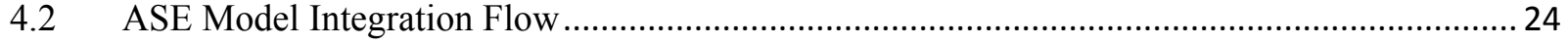

4.3 Filling in Missing Flight Data Points through Linear Interpolation..........................................24

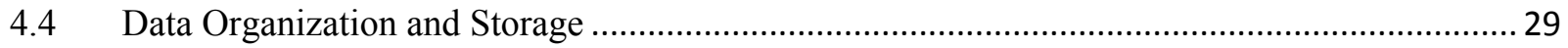

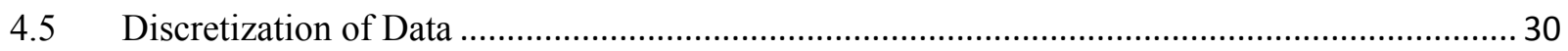

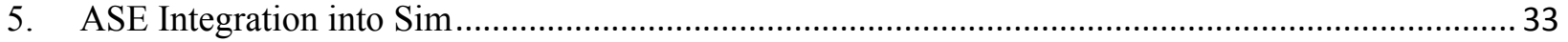




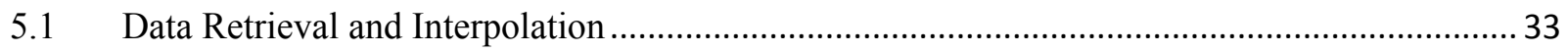

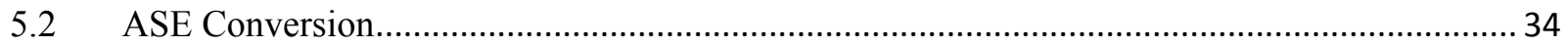

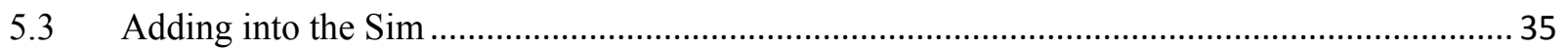

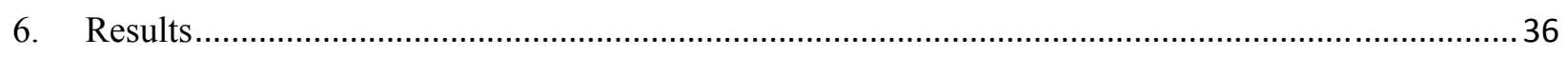

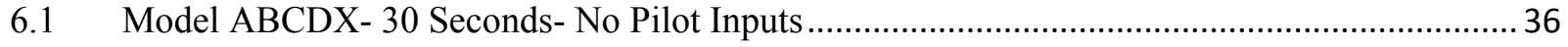

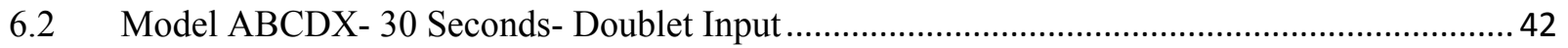

6.3 Model PLANTX- 30 Seconds- No Pilot Inputs .................................................................. 47

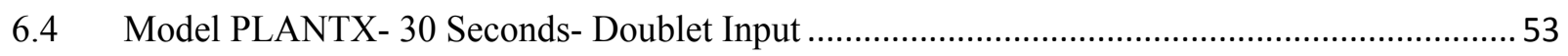

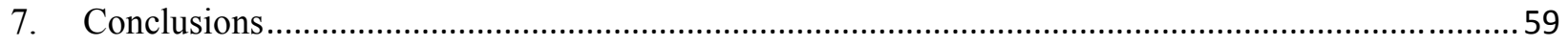

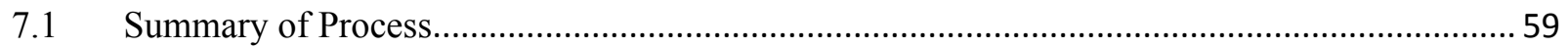

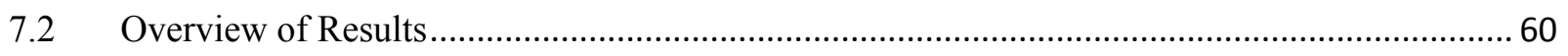

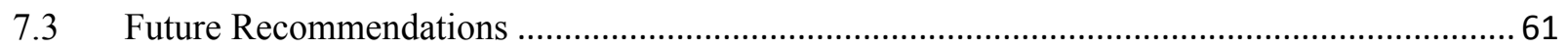

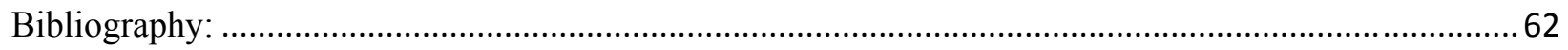

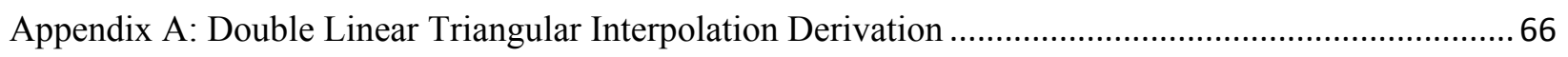

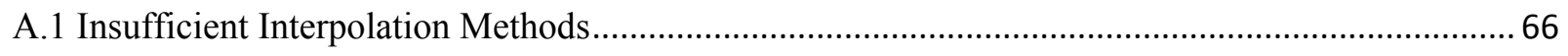

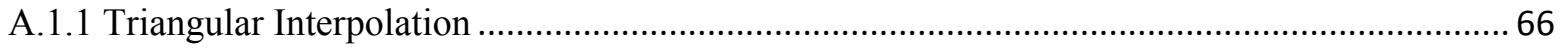

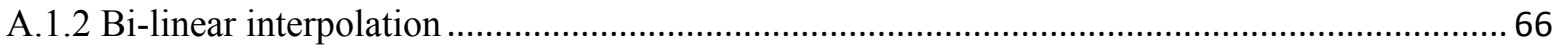

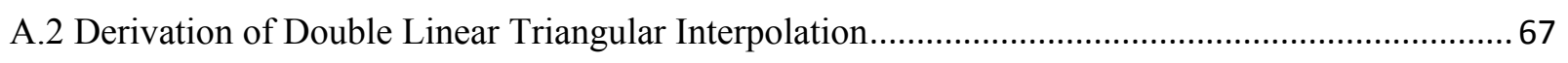

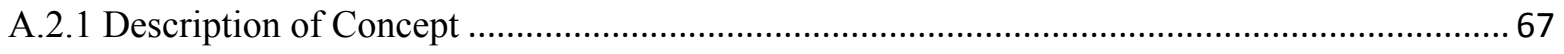

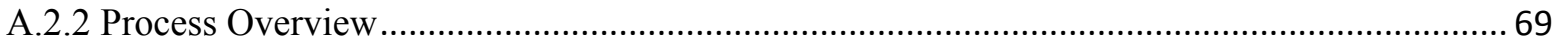

A.2.3 Derivation Equations-Planar Triangular Interpolation....................................................... 72

A.2.4 Derivation Equations-Fraction Determination .............................................................. 75

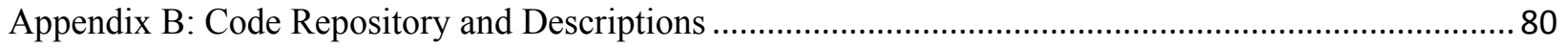

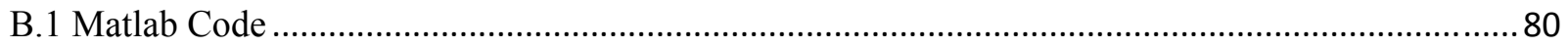

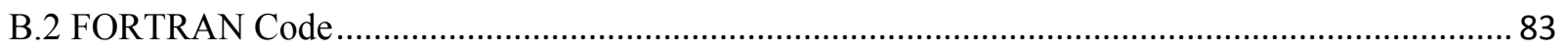




\section{Acronyms}

2D

$3 \mathrm{D}$

A

$A_{i}$

AAW

ABCDX

ACT

AFS

AIL

ASE

$B$

$B_{i}$

C

$C_{i}$

D

$D_{i}$

DAIL

DLEI

DLEO
Two Dimensional

Three Dimensional

low altitude $\&$ low mach point

A-matrix (state-space)

Active Aeroelastic Wing

ASE model without actuators

Active Control Technology

Active Flutter Suppression

Aileron

Aeroservoelastic/ Aeroservoelasticity

high altitude \& low mach point

B-matrix (state-space)

low altitude \& high mach point

C-matrix (state-space)

high altitude $\&$ high mach point

D-matrix (state-space)

Differential Aileron

Differential Leading Edge Inboard Flap

Differential Leading Edge Outboard Flap 


\begin{tabular}{|c|c|}
\hline DLTI & double linear triangular interpolation \\
\hline DOF & Degrees of Freedom \\
\hline DSTB & Differential Stabilizer \\
\hline DTEF & Differential Trialing Edge Flap \\
\hline $\mathrm{E}_{\mathrm{V}}$ & double linear triangular interpolated value \\
\hline $\mathrm{E}_{\mathrm{vx}}$ & interpolated value with respect to constant Mach- value \\
\hline $\mathrm{E}_{\mathrm{vxy}}$ & interpolated with respect to mach and altitude \\
\hline $\mathrm{E}_{\mathrm{x}}$ & flight altitude \\
\hline $\mathrm{E}_{\mathrm{y}}$ & flight mach \\
\hline $\mathrm{F} / \mathrm{A}$ & Fighter Aircraft \\
\hline $\mathrm{ft}$ & feet \\
\hline $\mathrm{Hz}$ & Hertz \\
\hline $\mathrm{K}$ & thousand \\
\hline LEF & Leading Edge Flap \\
\hline LEI & Leading Edge Flab- Inboard \\
\hline LEO & Leading Edge Flap- Outboard \\
\hline LTI & Linear Time Invariant \\
\hline MDO & Multidisciplinary Design and Optimization \\
\hline NASA & National Aeronautics and Space Administration \\
\hline NY & Body lateral acceleration \\
\hline NZ & Body normal acceleration \\
\hline $\mathrm{p}$ & Body axis roll rate \\
\hline Phi & Body roll attitude angle \\
\hline PLANTX & ASE model with actuators \\
\hline q & Body axis pitch rate \\
\hline r & Body axis yaw rate \\
\hline
\end{tabular}




\begin{tabular}{ll} 
rad & radian \\
RUD & Rudder \\
SAIL & seconds \\
Sim & Symmetric Aileron \\
SLEI & Simulator \\
SLEO & Symmetric Leading Edge Inboard Flap \\
STAB & Average Horizontal Stabilizer \\
STEF & Symmetric Trailing Edge Flap \\
TEF & Trailing Edge Flap \\
Theta & Body pitch attitude \\
$u_{k}$ & input vector \\
$\mathrm{v}$ & value \\
$\mathrm{x}$ & altitude \\
$x_{k}$ & state vector \\
$y_{n}$ & mach \\
\hline
\end{tabular}




\section{List of Figures}

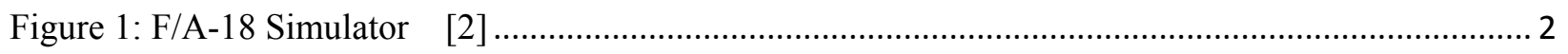

Figure 2: Collar's Aeroelastic Triangle [30] ................................................................................. 4

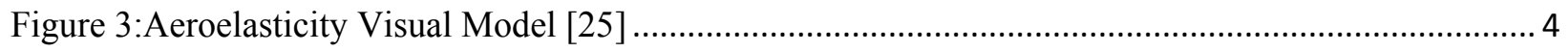

Figure 4: Aeroservoelastic Pyramid [1] …….......................................................................... 7

Figure 5: The Active Aeroelastic Research Plane [14] ..................................................................... 10

Figure 6: Active Aeroelastic Wing Airplane Schematic [16] .......................................................... 11

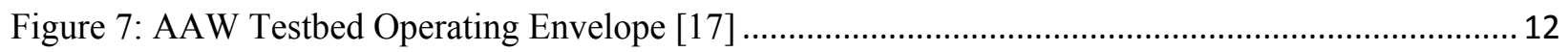

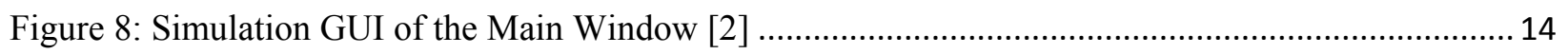

Figure 9: F/A-18 Simulation Flow Block Diagram- Rigid Body Dynamics Only ................................. 14

Figure 10: Simulation Flow Block Diagram- Rigid Body and ASE Dynamics....................................... 16

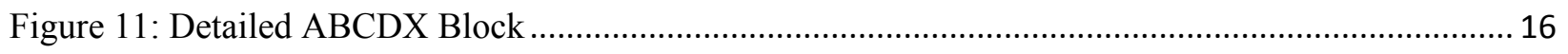

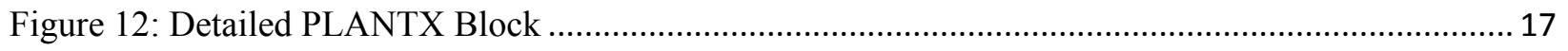

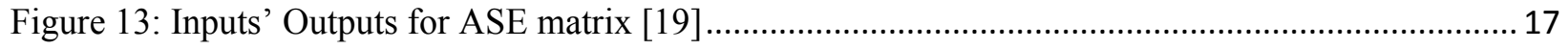

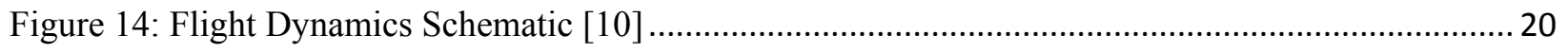

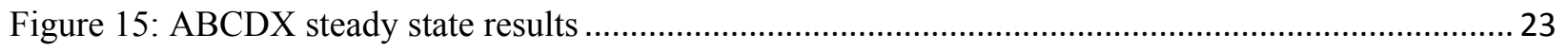

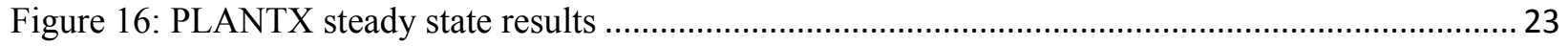

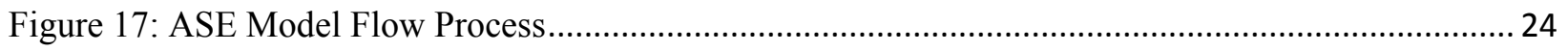

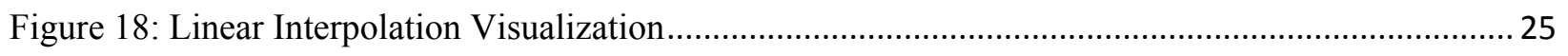


Figure 19: Initial Data Set.....

Figure 20: Linearly Interpolated Flight Condition Models .................................................................... 26

Figure 21: Copied ASE Models With Respect to Constant Mach ....................................................... 27

Figure 22: Linearly Interpolated Models at 30K Flight Condition .................................................... 27

Figure 23: Linearly Interpolated ASE Models With Respect to Constant Mach.....................................28

Figure 24: Complete ASE Baseline Model Set ............................................................................. 28

Figure 25: Representative Matrix Element of Baseline Matrix Plotted Throughout Entire Flight

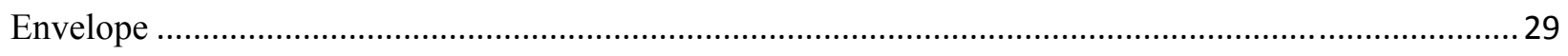

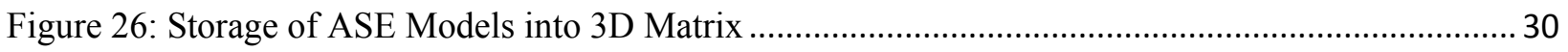

Figure 27: Zero Order Hold Concept Therefore, discretizing at $320 \mathrm{~Hz}$ shows a breakdown................... 31

Figure 28: Visual Schematic of Discretization of Data ................................................................... 32

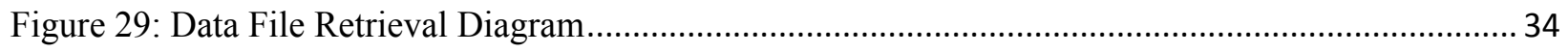

Figure 30: ABCDX Sim Steady State Plots- Flight Conditions …..................................................... 37

Figure 31: ABCDX Sim Steady State Plots- Rigid-Roll, Pitch, and Yaw Rate....................................... 37

Figure 32: ABCDX Sim Steady State Plots- Elastic Component-Roll, Pitch, and Yaw Rates................... 38

Figure 33: ABCDX Sim Steady State Plots- Elastic Component-Roll, Pitch and Yaw Rates-Percentage

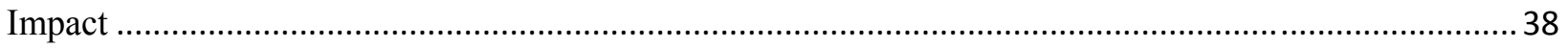

Figure 34: ABCDX Sim Steady State Plots- Rigid -Accelerations ...................................................... 39

Figure 35: ABCDX Sim Steady State Plots- Elastic Component- Accelerations ..................................... 39

Figure 36: ABCDX Sim Steady State Plots- Elastic Component- Accelerations- Percentage ................... 40

Figure 37: ABCDX Sim Steady State Plots- Rigid - Roll and Pitch Angle ........................................... 40

Figure 38: ABCDX Sim Steady State Plots- Elastic Component- Roll and Pitch Angles......................... 41

Figure 39: ABCDX Sim Steady State Plots- Elastic Component- Roll and Pitch Angles- Percentage...... 41

Figure 40: ABCDX Sim Steady State Plots -With Stick Doublet - Flight Conditions ............................ 42 
Figure 41: ABCDX Sim Steady State Plots- Rigid- Stick Doublet -Roll, Pitch, and Yaw Rate.

Figure 42: ABCDX Sim Steady State Plots- Elastic Component- Stick Doublet - Roll, Pitch, and Yaw

Rates. 43

Figure 43: ABCDX Sim Steady State Plots- Elastic Component-Roll, Pitch, and Yaw Rates- Stick

Doublet - Percentage Impact. .44

Figure 44: ABCDX Sim Steady State Plots- Rigid - Stick Doublet - Accelerations 44

Figure 45: ABCDX Sim Steady State Plots- Elastic Component- Stick Doublet - Accelerations ..... 45

Figure 46: ABCDX Sim Steady State Plots- Elastic Component- Stick Doublet - Accelerations-

Percentage. .45

Figure 47: ABCDX Sim Steady State Plots- Rigid - Stick Doublet - Roll and Pitch Angle 46

Figure 48: ABCDX Sim Steady State Plots- Elastic Component- Stick Doublet - Roll and Pitch Angles 46 Figure 49: ABCDX Sim Steady State Plots- Elastic Component- Stick Doublet - Roll and Pitch Angles-

Percentage. 47

Figure 50: PLANTX Sim Steady State Plots- Flight Conditions...... 48

Figure 51: PLANTX Sim Steady State Plots- Rigid- Roll, Pitch, and Yaw Rate 48

Figure 52: PLANTX Sim Steady State Plots- Elastic Component- Roll, Pitch, and Yaw Rates 49

Figure 53: PLANTX Sim Steady State Plots- Elastic Component- Stick Doublet - Roll, Pitch, and Yaw

Rates-Percentage Impact. 49

Figure 54: PLANTX Sim Steady State Plots- Rigid -Accelerations ..... 50

Figure 55: PLANTX Sim Steady State Plots- Elastic Component- Accelerations 50

Figure 56: PLANTX Sim Steady State Plots- Elastic Component- Accelerations- Percentage ................. 51

Figure 57: PLANTX Sim Steady State Plots- Rigid - Roll and Pitch Angle 51

Figure 58: PLANTX Sim Steady State Plots- Elastic Component- Roll and Pitch Angles 52

Figure 59: PLANTX Sim Steady State Plots- Elastic Component- Roll and Pitch Angles- Percentage .... 52 
Figure 60: PLANTX Sim Steady State Plots- Flight Conditions

Figure 61: PLANTX Sim Steady State Plots- Rigid- Stick Doublet - Roll, Pitch, and Yaw Rate..... .54

Figure 62: PLANTX Sim Steady State Plots- Elastic Component- Stick Doublet - Roll, Pitch, and Yaw

Rates. .54

Figure 63: PLANTX Sim Steady State Plots- Elastic Component-Roll, Pitch, and Yaw Rates- Stick

Doublet - Percentage Impact. 55

Figure 64: PLANTX Sim Steady State Plots- Rigid - Stick Doublet - Accelerations...... 55

Figure 65: PLANTX Sim Steady State Plots- Elastic Component- Stick Doublet - Accelerations .56

Figure 66: PLANTX Sim Steady State Plots- Elastic Component- Stick Doublet - Accelerations-

Percentage. .56

Figure 67: PLANTX Sim Steady State Plots- Rigid - Stick Doublet - Roll and Pitch Angle..... 57

Figure 68: PLANTX Sim Steady State Plots- Elastic Component- Stick Doublet - Roll and Pitch

Angles .57

Figure 69: PLANTX Sim Steady State Plots- Elastic Component- Stick Doublet - Roll Angles-

Percentage. .58

Figure 70: Triangular Interpolation Assumes All Four Data Points are Co-Planar..... 66

Figure 71: Bi-linear Interpolation is a Quadratic Interpolation Scheme... 67

Figure 72: Triangular Prism. 68

Figure 73: Diagram of Four Planes 68

Figure 74: 2D Plane Intersection 69

Figure 75: Base Line Cutting Through Two Planes of the Triangular Prism ....... 69

Figure 76: Average Plane Between Planar Edges. 70

Figure 77: 2D Plane Assignment Breakdown 71

Figure 78: Determining limit line based on fraction of Mach and Altitude. 71 
Figure 79: Determining limit line based on fraction of Mach and Altitude

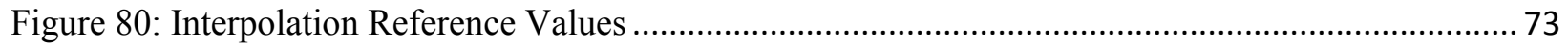

Figure 81: Plane 1 Reference Table and Schematic....................................................................... 73

Figure 82: Plane 2 Reference Table and Schematic...................................................................... 74

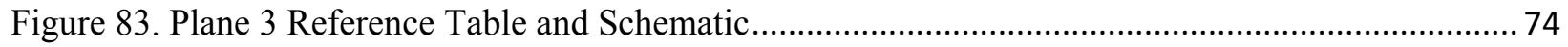

Figure 84. Plane 4 Reference Table and Schematic....................................................................... 75

Figure 85: a) Triangular Interpolation (b) Bilinear Interpolation (c) DLTI........................................ 78

Figure 86: Example Matrix Element Using Double Linear Interpolation Scheme Through All Flight

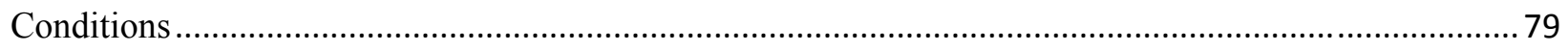

Figure 87: computation process in F18 Sim (Flow diagram of processes) …...................................... 84

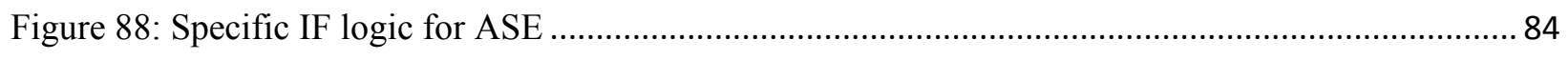

Figure 89: Flow Diagram Highlighting Location of FORTRAN Files for Sim....................................... 85 


\section{List of Tables}

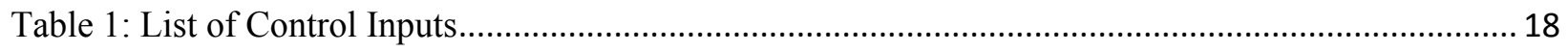

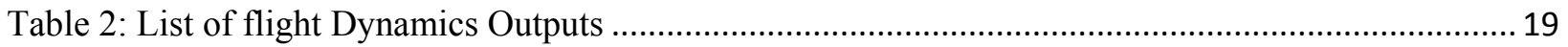




\section{Chapter 1}

\section{Introduction and Purpose}

Aircraft are elastic structures that are subject to deformation in both static and dynamic conditions [1]. Effects from aeroelasticity create dynamic loads that can directly affect the shape and stability of various aircraft structures. In addition, the interaction between an aircraft's flight control systems and aeroelastic influences can adversely affect the flight dynamics of aircraft leading to unexpected dynamic interactions and flight responses.

In high speed aircraft such as the F/A-18, elastic structural properties must be accounted for to ensure no undesirable structural dynamic effects occur throughout its flight. Typically, elastic structural modes are identified and filtered out of the flight dynamics through notch filtering of sensor data [1]. However, as modern aircraft design demands lighter and more flexible structures, it is becoming essential to create complete and accurate models of aircraft with both rigid body and elastic dynamic properties. Therefore, to better characterize elastic effects on aircraft during flight, aeroservoelastic (ASE) structural models were created using flight data from the Active Aeroelastic Wing (AAW) program [15].

These models were added to the F/A-18 simulator at the NASA Dryden Flight Research

Center. The NASA Dryden F/A-18 flight simulator is a complex research platform used to study the flight characteristics of the F/A-18 in flight as shown in Figure 1. 


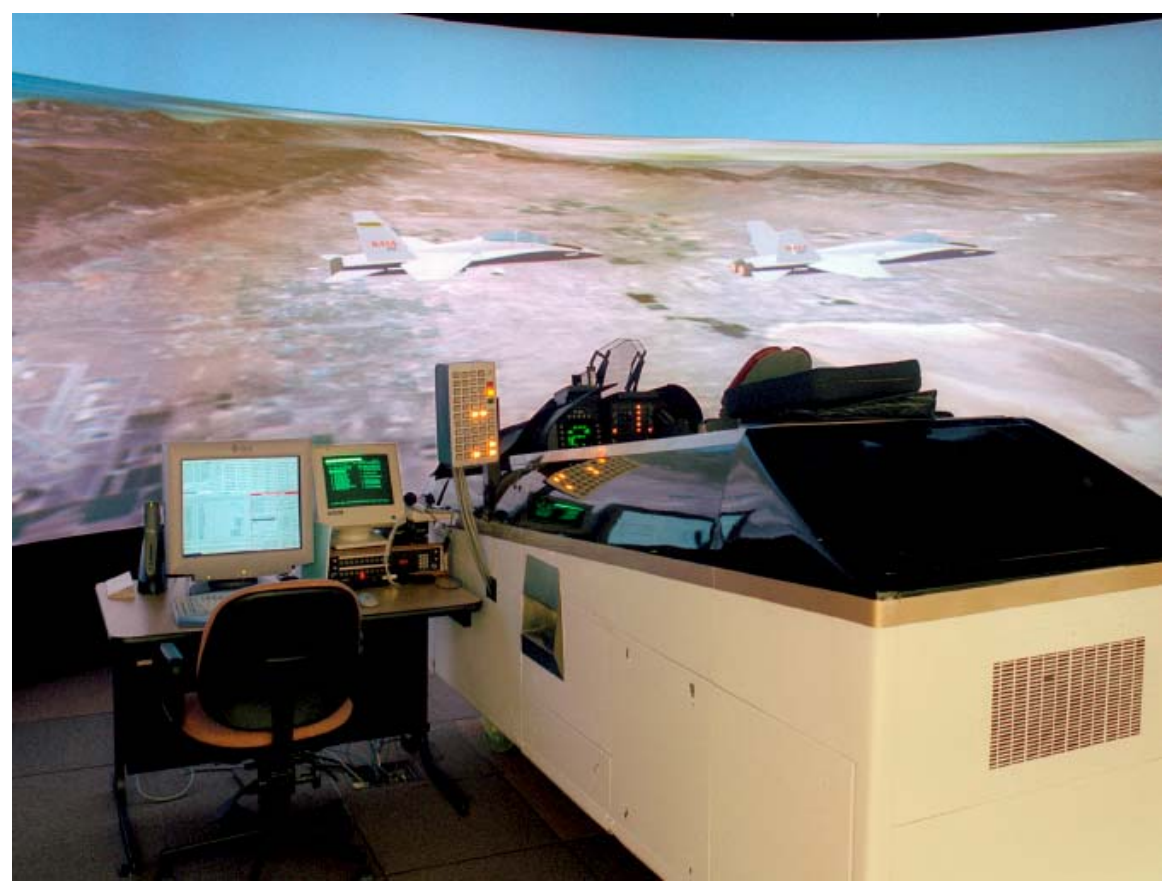

Figure 1: F/A-18 Simulator [2]

The ASE models were integrated in parallel to the flight dynamics simulation equations to minimize disruption to its already functioning operating framework. Demonstration of ASE integration into the F/A-18 simulator is a first step to better understanding simulation models of aircraft with both rigid body and elastic components. This process can provide a reference for the future development of combined rigid and elastic models for other aircraft as these united models are better able to characterize the true flight dynamics of aircraft. 


\section{Chapter 2}

\section{Background}

\subsection{Aeroelasticity}

In aircraft, dynamic loads come from interactions between the airplane structure and the airflow around the plane. The science which studies the mutual interaction between aerodynamic, inertial, and elastic forces in aircraft is called aeroelasticity $[3,4]$. The interaction of these forces is characterized by the triangle of interacting forces in Figure 2 from Arthur Collar [30]. 


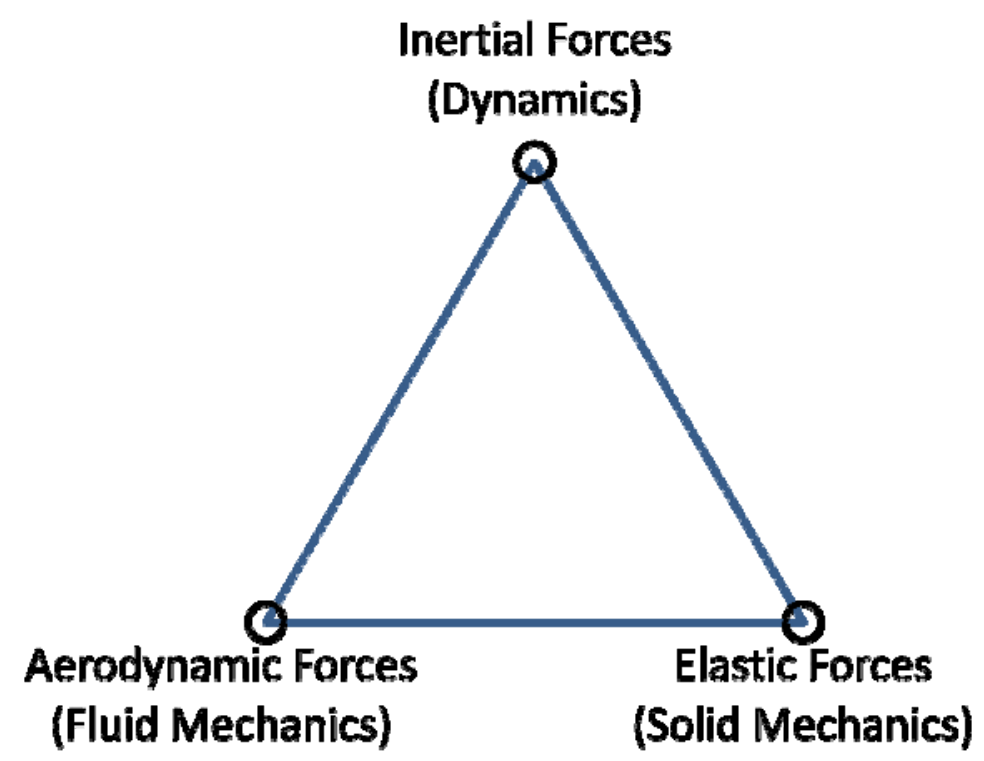

Figure 2: Collar's Aeroelastic Triangle [30]

Phenomena arising from aeroelasticity derive from airplane structures that are not perfectly rigid. In fact, modern airplane structures have varying levels of flexibility and elasticity. A visual schematic of a flexible airplane structure is shown in Figure 3 [5].

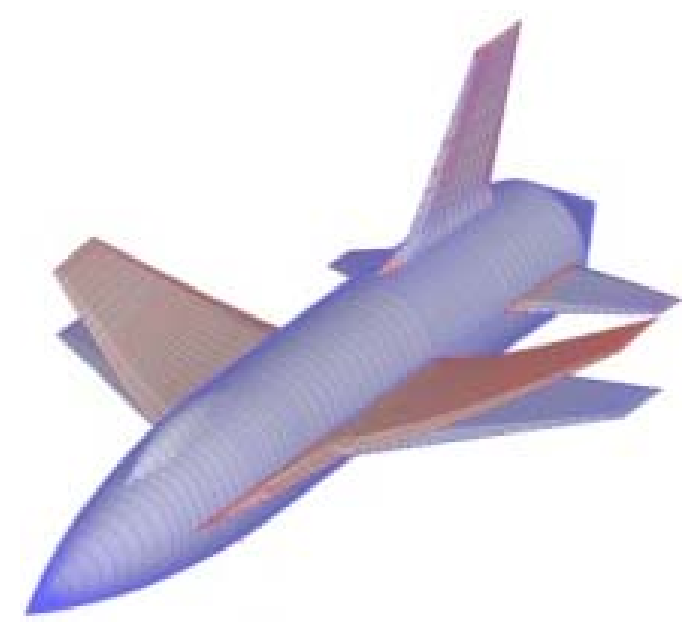

Figure 3:Aeroelasticity Visual Model [25]

In general, initial designs often assume that the external loading acting on a body is independent of the deformation of the body. This is because the deformation is small and does not substantially affect the action of the external forces. Therefore force calculations are based 
on the initial shape of the body, neglecting the changes in dimensions of the body. In aeroelasticty the aerodynamic forces depend critically on the attitude of the body relative to the flow. The elastic deformation plays a significant role in determining the external loading itself. The magnitude of the aerodynamic force is not known until the elastic deformation is determined. As a result, the external load is not known until the elasticity problem is fully solved [6]. Ignoring aeroelasticity may lead to poor assumptions causing adverse flight responses for the airplane.

The field of aeroelasticity came to prominence after World War II. Prior to this time, airplanes traveled at relatively low speeds allowing the structure to stay sufficiently rigid to avoid most aeroelastic effects. However, as airplanes increased in speed, without additional structural and loads support, aircraft designers encountered a wide array of problems that are now classified as aeroelastic problems [5].

A summary of the effects of aeroelasticity on the design of aircraft are listed below:

Buffeting: Rough transient vibrations in the tail due to aerodynamic impulses from the wake of the wing $[5,7]$.

Dynamic Loads Problems: Added loads from aerodynamic induced bending and torsional stresses in the wing and fuselage beams during flight [5].

Load Redistribution: Aircraft structure deflection tends to redistribute the air loads, and may cause the load distribution on the structure to be significantly different from that computed on the assumption of complete rigidity. Therefore, engineers must ensure that the structural members are designed for air load distributions corresponding to the deformed structure [5]. 
Divergence: Primarily in aircraft wings, when the aircraft speed exceeds the torsional divergence speed, the increment in aerodynamic torsional moment exceeds the increment in elastic restoring torque, causing the wing to become statically unstable [5].

Control Effectiveness and Reversal: Aircraft with conventional platforms may suffer serious loss of aileron, elevator, and rudder control effectiveness due to elastic deformations of the structure. In some cases, this can lead to reverse effects to control commands [5].

Flutter: Flutter phenomena occur as a self-induced and potentially destructive vibration when the interaction between aerodynamic forces and a structure couple with the structure's natural mode of vibration to produce a divergent oscillation. The structure extracts energy from the air stream feeding an unstable self-excited vibration which can result in catastrophic structural failure $[1,6,8]$.

In most cases aeroelastic problems are solved by increasing the stiffness of varying parts of the airplane structure. However, this can be a costly process at the expense of adding considerable weight.

\subsection{Aeroservoelasticity (ASE)}

Aeroservoelasticity takes the study of aeroelasticity one step further by considering the interaction between aerodynamics, inertial, structural, actuation, and control system dynamics [9]. This relationship is shown in the aeroservoelastic pyramid in Figure 4. 


\section{Inertial Forces}

(Dynamics)

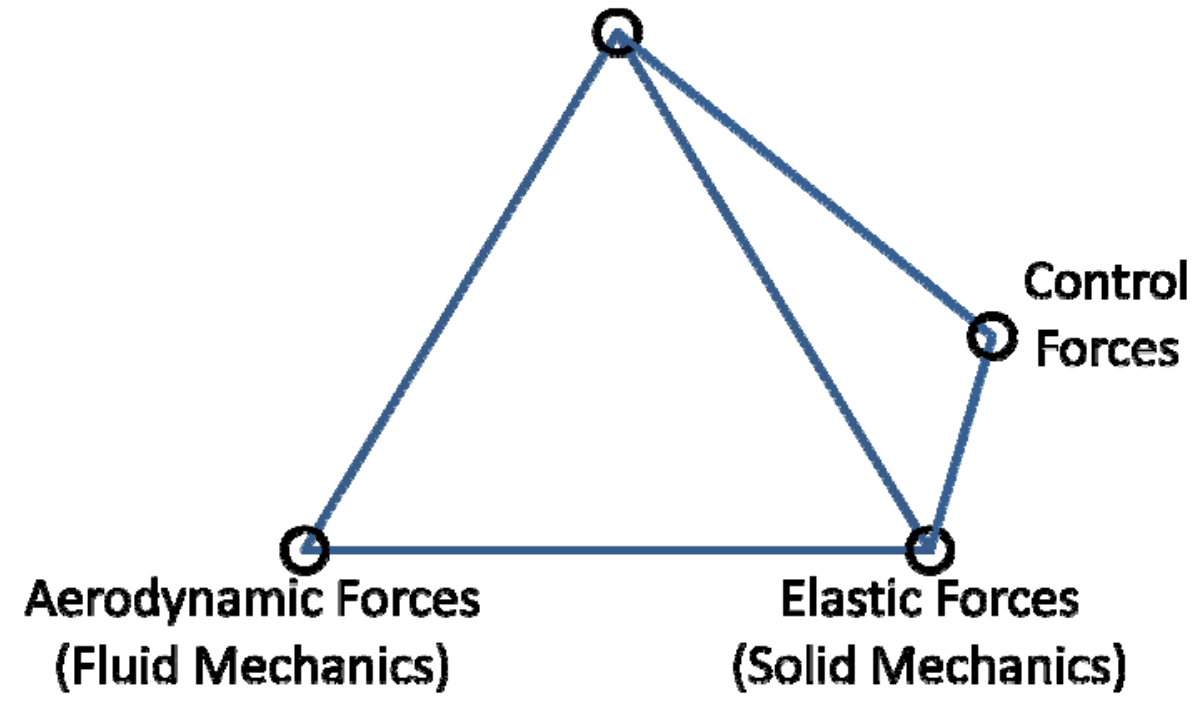

Figure 4: Aeroservoelastic Pyramid [1]

The study of aeroservoelasticity came into prominence with the development of active controls using high gain feedback loop control systems [28,29]. Such control systems have become powerful enough to influence the dynamic stability and structural response of the aircraft structure. Consequently, adverse reactions can develop between the control system and the elastic structural dynamics [10].

Sensors throughout the airplane provide information to the control system on how effective the control surfaces are influencing the plane's motion. However, some control system sensors such as gyros and accelerometers, not only sense rigid body motion, but elastic mode motion as well. As a result, control surfaces may deflect in response to sensor signals from elastic structural modes. This can either dampen or amplify structural vibrations. In the case where structural vibrations are amplified, a self-excited oscillation can occur and result in a flutter phenomenon similar to effects from aeroelasticity. However, this form of flutter derives part of its energy from the control system itself. Therefore, the control system must be carefully 
designed to account for aerodynamics, actuator responses, sensor information, and structural dynamics in order to be able to properly control aeroservoelastic phenomena [10].

In order to fully analyze an aeroservoelastic system, one must understand the multi-input multi-output sampled-data control feedback with actuation dynamics coupled with aeroelasticity. This can make discrimination between the source and responses of components affecting aeroservoelasticity difficult to determine [11]. Thus in order to be able to distinguish system component dynamics and determine the expected response of an aircraft in flight, structural, aerodynamic, and control system disciplines can no longer be treated independently and must be studied together to fully understand the phenomena associated with aeroservoelasticity [10,22].

Notch filters have traditionally been included into control systems to eliminate any aeroservoelastic interactions. However, as modern aircraft have increasingly stringent performance requirements and development of intentional structurally flexible materials with active flutter suppression (AFS) or active control technology (ACT) increases, aeroservoelastic dynamic analysis becomes increasingly important $[8,31]$.

The combined fields of aeroelasticity and aeroservoelasticity working with multidisplinary optimization (MDO) will play a major role in the development, design, and analysis of variable shaped planes. Advances in structural dynamics and materials technology can potentially lead to various levels of morphing vehicles [26]. These developments may revolutionize aircraft designs with drastic increases in fuel efficiency and vehicle maneuverability.

\subsection{Active Aeroelastic Wing (AAW) Program}

The development of highly flexible vehicles requires the use of active control systems that demand significant interaction with aero and structural dynamics [28]. Using aeroelasticity 
as phenomena that can be controlled instead of avoided sparked research into the potential development of a new concept in aircraft design [27]. In 1996, the Air Force Research Laboratory and NASA initiated the Active Aeroelastic Wing (AAW) project (later assigned the designation of "X-53") to investigate the use of wing twist for roll control. The AAW concept was tested on a modified F/A-18 supersonic fighter aircraft during the spring of 2005 [12]. The AAW program had wings modified for reduced torsional stress that required the development of tools to concurrently integrate control and structural design. This allowed savings in structural weight and drag, improvements in cruise and roll performance, increases in fatigue life, and increased design latitude in terms of wingspan, sweep, and thickness [13].

\subsubsection{Vehicle Selection}

A NASA Dryden F/A-18A airplane was selected as the AAW research plane for three primary reasons: First, NASA already had a knowledgeable team about the airplane and its systems, including a fully developed F/A-18 simulation set up. Second, the F/A-18 leading-edge flap (LEF) was already split into inboard and outboard sections, allowing for a modification to allow the inboard section of the LEF to operate independently of the outboard section. Having an additional set of control surfaces improved the ability to control the twist, aerodynamics, and load distribution of the wing from the leading-edge flaps. Third, during preproduction performance of the F/A-18 airplane, the wings were found to be too flexible and did not meet desired roll performance criteria. This caused the designers to redesign the F/A-18 with a stronger and heavier wing. Therefore, the preproduction plane provided a history where wing flexibility was detrimental to aircraft performance and the AAW program can better demonstrate any flight control improvements from the technology on the same plane [14]. The aircraft is shown in Figure 5. 


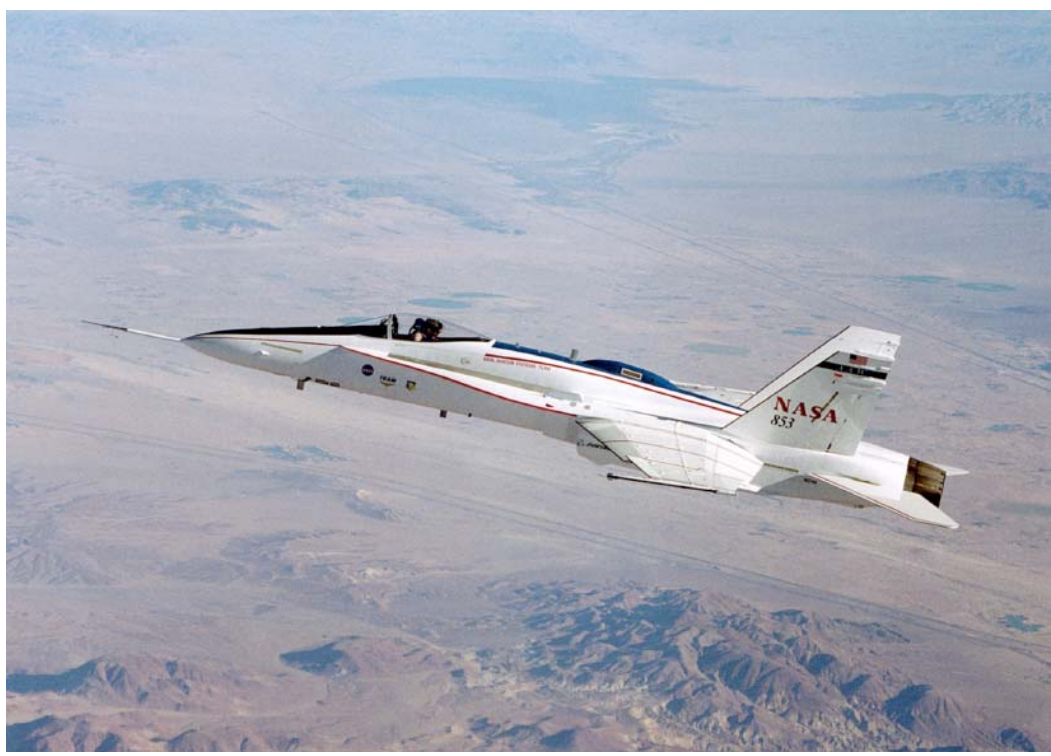

Figure 5: The Active Aeroelastic Research Plane [14]

\subsubsection{Vehicle Modifications}

The AAW F/A-18 used wings that returned to the stiffness levels of the pre-production F/A-18 design to provide the greater flexibility needed for flight testing. The flap control surface, both inboard and outboard were separated from operating as a single unit, and instead operated independent from one another [26]. This allowed the LEF's a greater range of motion and control of the airplane. In addition, the AAW flight control computers were modified to operate with the more flexible wing, while still maintaining the ability to switch with the standard F/A-18 control laws as needed. Multiple sensors were added to ensure all data was collected to meet flight objectives for analysis [14]. A schematic of the major control surfaces is shown in Figure 6. 


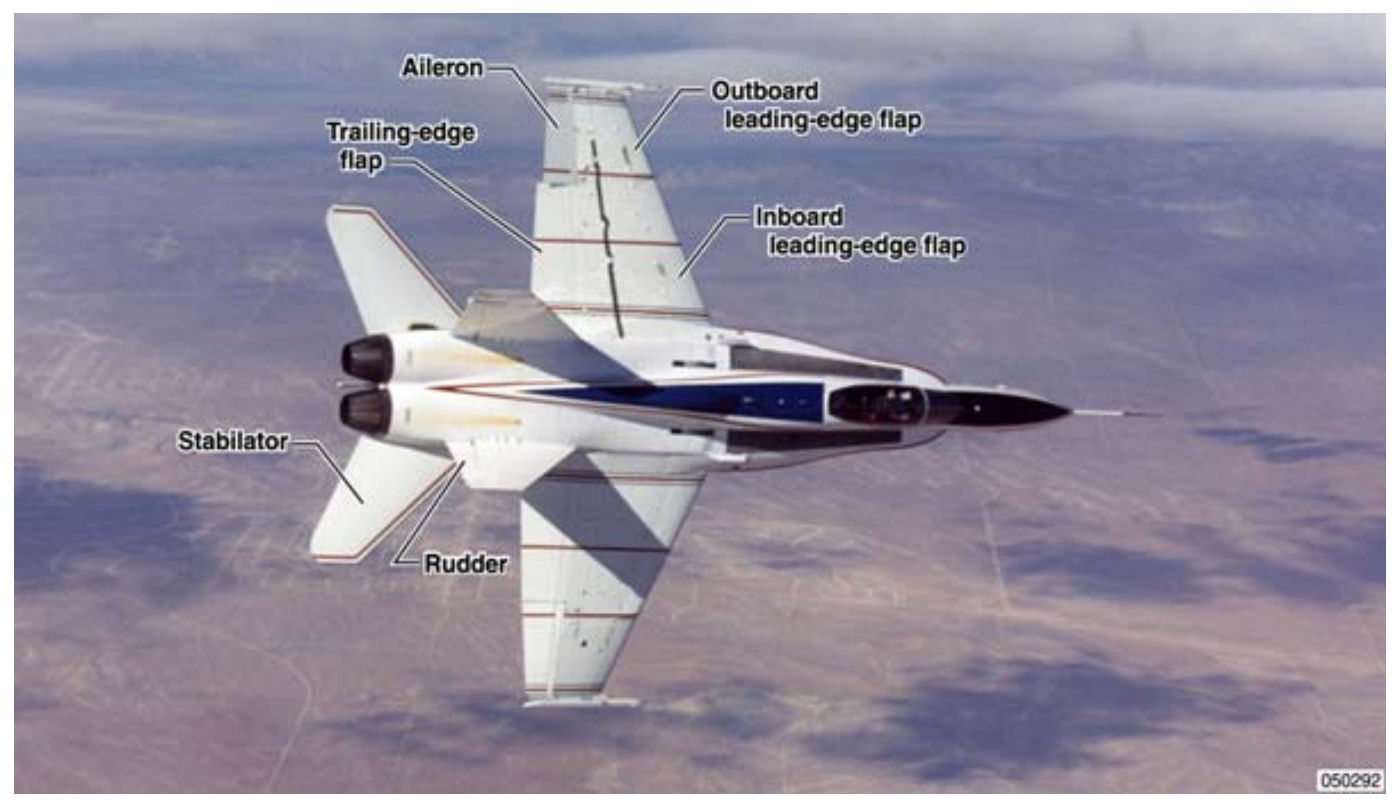

Figure 6: Active Aeroelastic Wing Airplane Schematic [16]

The AAW technology is multidisciplinary and integrates technologies in air vehicle dynamics, active controls, and structures to maximize air vehicle performance. Instead of treating aeroelastic phenomena as a hindrance, the AAW uses wing flexibility as a benefit to optimize aircraft control using high aspect ratio, thin, swept fighter wings that are aeroelastically deformed. Design studies where this technology is applied has shown significant weight and performance benefits and is considered by some engineers to be the next step in the evolution of wing design [15].

The purpose of the AAW program was to prove that an F/A-18 can be controlled using a flexible wing as a non-conventional control surface. The goal was to demonstrate improved aircraft roll control through aerodynamically induced wing twist on a full scale high performance aircraft at transonic and supersonic speeds [16]. Data from the program include the measurements of the change in aircraft speed, acceleration, and orientation as a response from control system inputs. The AAW program captured several different flight condition test points to characterize the flight test point envelopes as shown in Figure 7. 


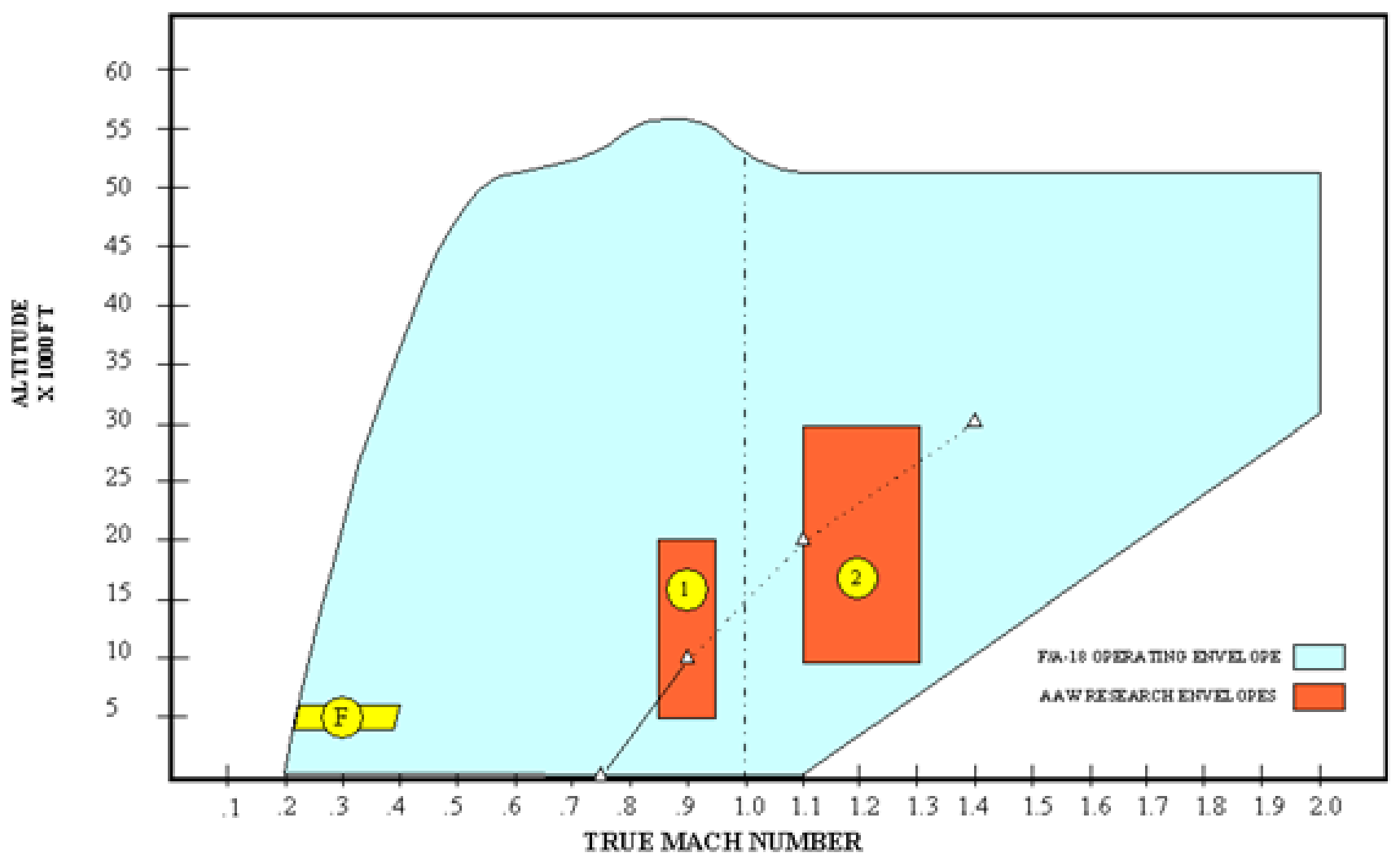

Figure 7: AAW Testbed Operating Envelope [17]

\subsection{F/A-18 Simulator}

\subsubsection{F/A-18 Simulator Background}

The NASA Dryden Flight Research Center has developed a reliable and robust simulation software package for a broad range of fixed-wing aircraft, including the F/A-18. The software structure allows for use in a batch-mode, real-time pilot-in-the-loop, and flight hardware-in-the-loop simulation. Currently the F/A-18 simulator is used more for engineering analysis than for pilot training. Typical simulation tasks include evaluation of new vehicle concepts, control law development and validation, flight safety analysis, mission planning, flight envelope expansion, and post flight data analysis [2]. 
The F/A-18 simulation operates on UNIX-based platforms and is primarily coded using a FORTRAN shell and C support routines as languages [18]. Most of the code is written in FORTRAN which includes the aircraft models, equations of motion, integration, table look-ups, initialization, and display generation routine, while $\mathrm{C}$ is used for the graphical user interface (GUI) and system specific routines such as memory mapping, priority boosting, and interrupt handlers [18].

FORTRAN language is still used because of the significant amount of FORTRAN code retained from earlier simulation iterations. However, the FORTRAN code is shifting toward C as older code becomes obsolete [18]. To minimize disruption with the simulator and to maintain consistency with the other loads definition script files, the ASE code was also written in the FORTRAN programming language to allow for direct integration into the simulator with the existing framework.

The simulation is compiled using UNIX "makefiles." This allows for minor updates to the sim to be made in a short amount of compiling time (well under 5 minutes) [18].

\subsubsection{Simulator Calculation Process}

The main simulation program is referred to as the background executive. This FORTRAN task is used to initialize the simulation database and provide a display and command line interface for monitoring and controlling the simulation. Once this main program has performed its initialization function, it starts the real-time loop as a separate task. The real-time loop may contain one or more programs running in parallel. This parallelism is used as needed to meet frame-time requirements. For batch mode, only one pseudo real-time loop exists that runs the models in series [2]. This allows for large data base references to only load once at the 
beginning, saving time during the running of the simulation and keeping the simulation in real time [18]. The F/A-18 simulator user interface is shown below in Figure 8.

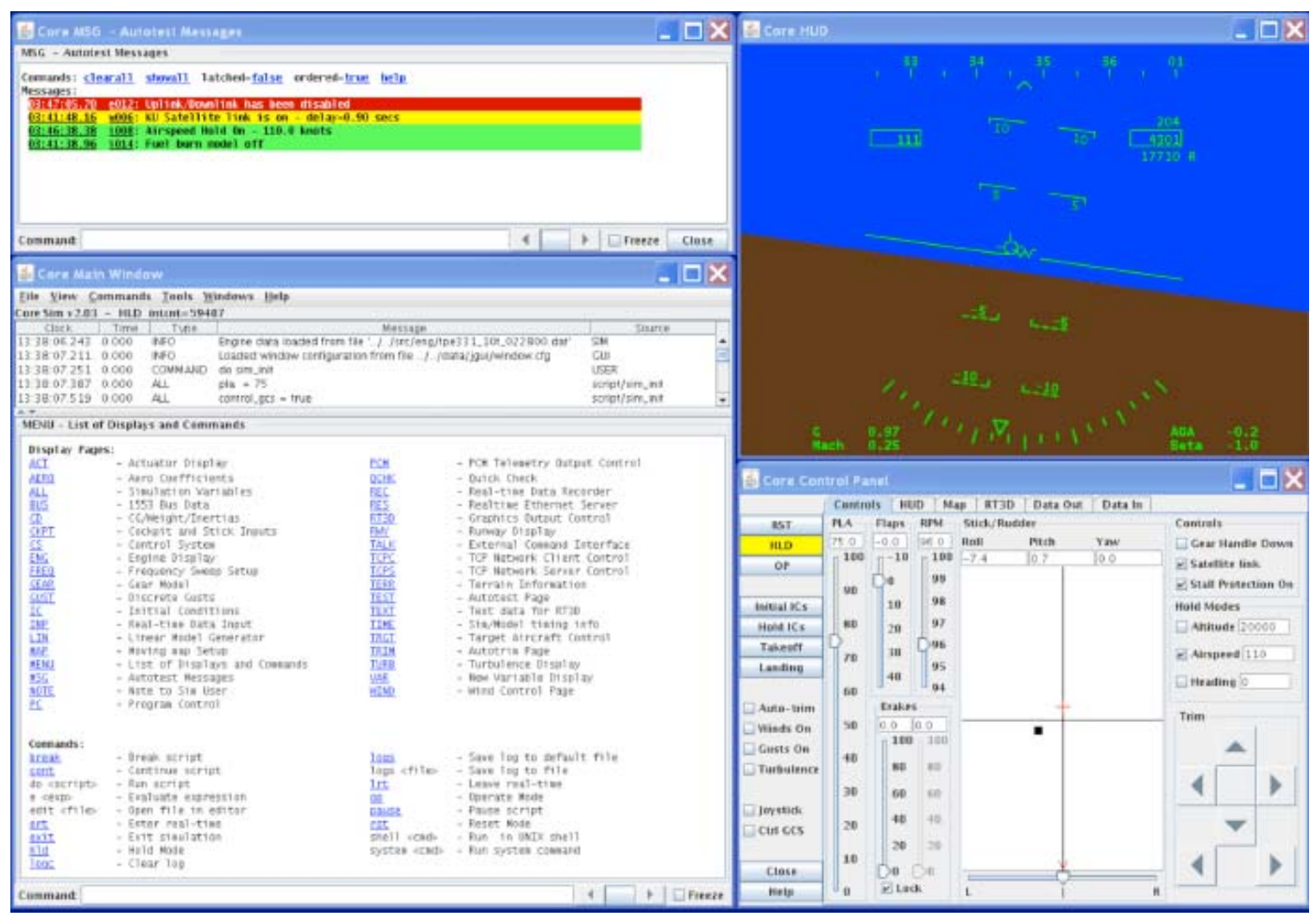

Figure 8: Simulation GUI of the Main Window [2]

Interface displays include the Main Window, visual HUD, cockpit control panel, and flight map (not shown). Currently, the F/A-18 Simulator is run through the simplified diagram depicted in Figure 9:

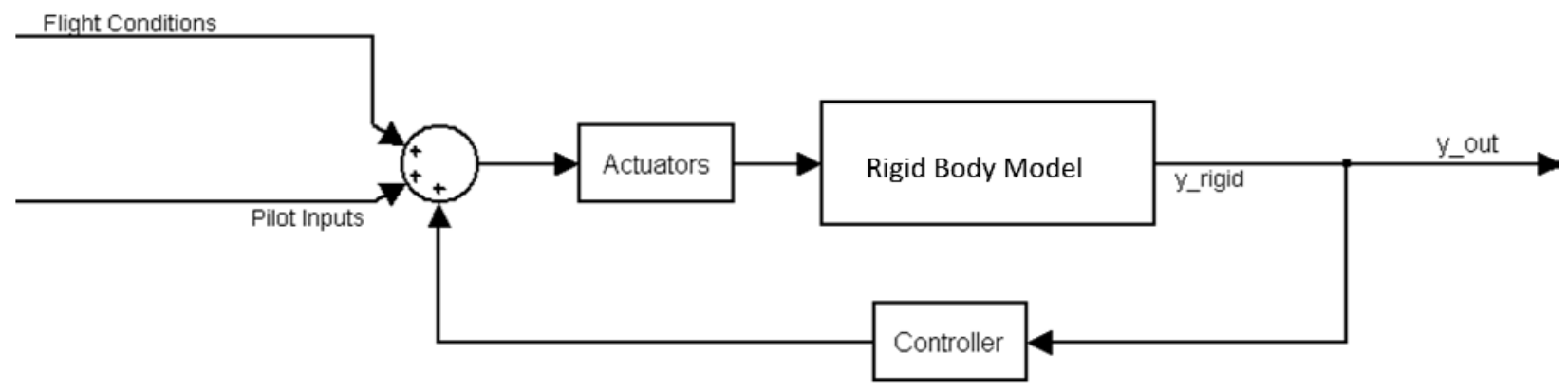

Figure 9: F/A-18 Simulation Flow Block Diagram- Rigid Body Dynamics Only 


\section{Chapter 3}

\section{Integration of ASE Models into Simulator}

\subsection{Integration Technique}

The ASE properties were added in parallel with the rest of the sim to ensure minimal interference of normal operations. Additionally, running the ASE analysis routine in parallel with the rest of the sim, will allow on and off capabilities of the ASE model without effecting the normal operation of the sim.

Two types of models were integrated into the sim: One model did not have actuator models included and was designated model 'ABCDX', and the other model did have actuator models included and was given the designation of 'PLANTX'. This would affect the type of inputs into the ASE models. The overall flow diagram is shown in figure 10. The purpose of integrating two different ASE models is to ensure flexibility in operating the simulation. The PLANTX model, which has internal actuator models included, is the desired ASE model to use since it better characterizes the coupled interaction between the actuators and the aeroelastic model. However, this model is over twice as large as the ABCDX model, which only contains the aeroelastic model. As a result, there was initial concern that integration of PLANTX may not be possible while keeping the entire simulation running in real time. Therefore, the capability is 
needed to also run $\mathrm{ABCDX}$ to at least still have the ability to add elastic properties to the sim in real time. An additional benefit is having the capability to compare the outputs of two different elastic models connected at different locations in the simulator to verify their accuracy after integration.

\section{ASE Inputs}

Matrix: $A B C D X$, Inputs actuator positions

- - - - Matrlx: PLANTX, Inputs actuator commands

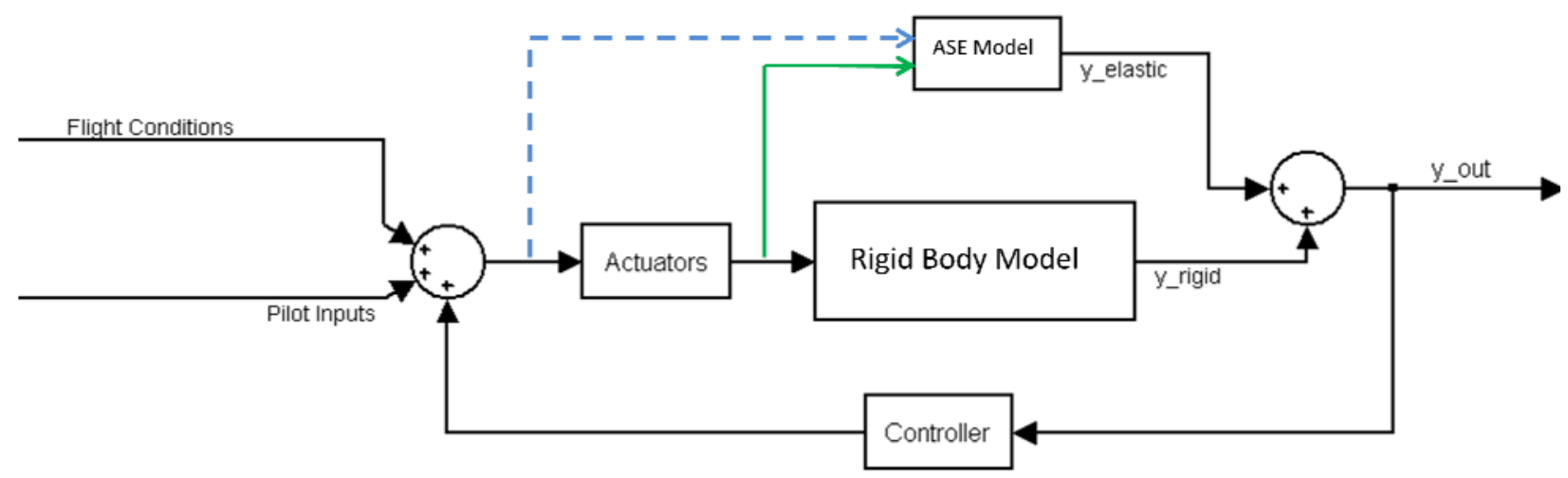

Figure 10: Simulation Flow Block Diagram- Rigid Body and ASE Dynamics

Data set ' $\mathrm{ABCDX}$ ' does not contain actuator data and would thus be hooked up after the existing actuator models as shown in Figure 11:

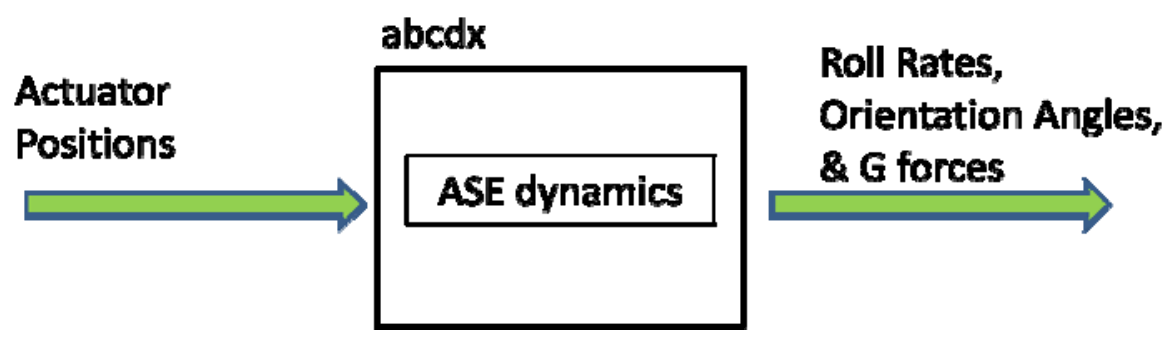

\section{Figure 11: Detailed ABCDX Block}

Data set 'PLANTX' contains the actuator models and can be hooked up with the inputs directly from the actuator commands as shown in Figure 12: 


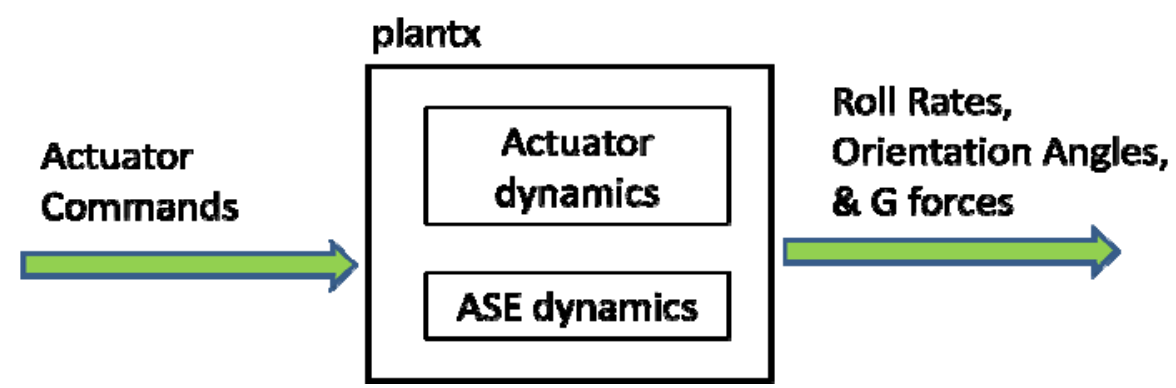

Figure 12: Detailed PLANTX Block

Total inputs and outputs of ASE model in the simulator are shown below in figure 13:

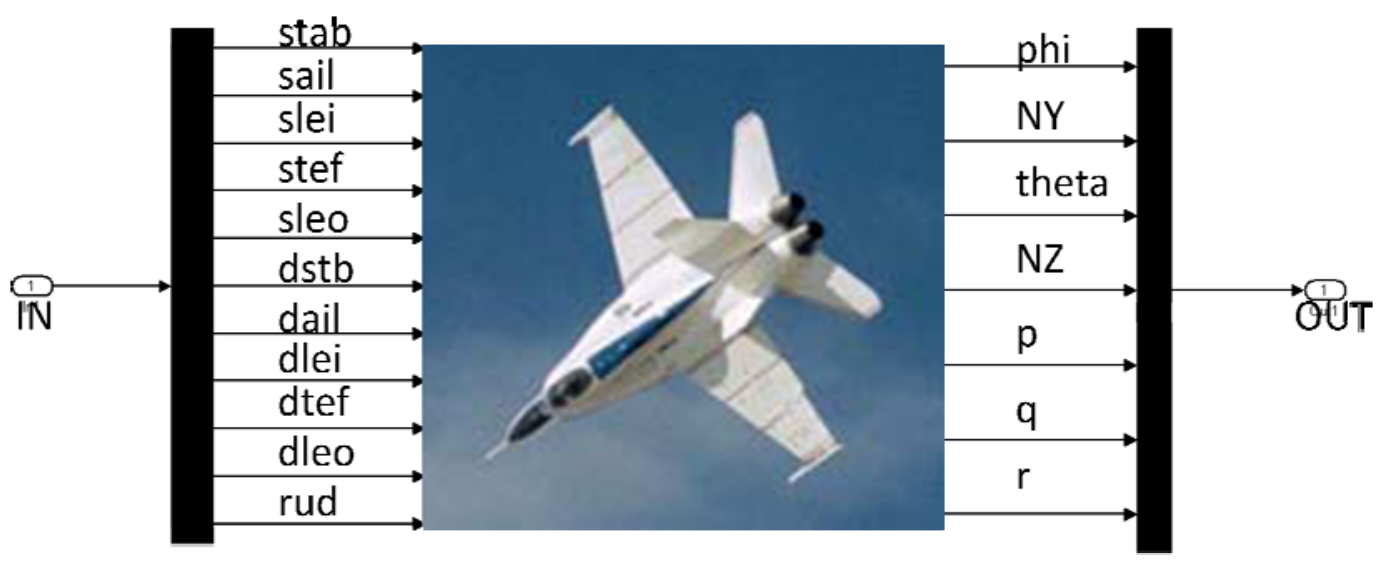

Figure 13: Inputs' Outputs for ASE matrix [19]

The input controls are listed and described in detail in table 1. 
Table 1: List of Control Inputs

\begin{tabular}{|l|l|}
\hline Controller & Description \\
\hline STAB & average horizontal stabilizer \\
\hline SAIL & symmetric aileron \\
\hline SLEI & symmetric leading edge inboard flap \\
\hline STEF & symmetric trailing edge flap \\
\hline SLEO & symmetric leading edge outboard flap \\
\hline DSTB & differential stabilizer \\
\hline DAIL & differential aileron \\
\hline DLEI & differential leading edge inboard flap \\
\hline DTEF & differential trailing edge flap \\
\hline DLEO & differential leading edge outboard flap \\
\hline RUD & rudder \\
\hline
\end{tabular}

Inputs were defined by the symmetric and differential deflections of the control surfaces shown below [14]:

Symmetric deflections:

$$
\begin{gathered}
s_{A I L}=\delta_{A I L}=0.5\left(\delta_{A I L_{L e f t}}+\delta_{A I L_{\text {Right }}}\right) \\
s_{S T A B}=\delta_{S T A B}=0.5\left(\delta_{S T A B_{\text {Left }}}+\delta_{S T A B_{\text {Right }}}\right) \\
S_{T E F}=\delta_{T E F}=0.5\left(\delta_{T E F_{\text {Left }}}+\delta_{\text {TEF }}\right. \text { Right } \\
S_{L E I}=\delta_{L E I}=0.5\left(\delta_{L E I_{\text {Left }}}+\delta_{L E I_{\text {Right }}}\right) \\
S_{L E O}=\delta_{L E O}=0.5\left(\delta_{L E O_{\text {Left }}}+\delta_{L E O_{\text {Right }}}\right)
\end{gathered}
$$




$$
S_{R U D}=\delta_{R U D}=0.5\left(\delta_{R U D_{L e f t}}+\delta_{R U D_{R i g h t}}\right)
$$

Differential deflections:

$$
\begin{gathered}
d_{A I L}=\delta_{d A I L}=\delta_{A I L_{\text {Left }}}-\delta_{A I L_{\text {Right }}} \\
d_{S T A B}=\delta_{d S T A B}=\delta_{S T A B_{\text {Left }}}-\delta_{S T A B_{\text {Right }}} \\
d_{T E F}=\delta_{d T E F}=\delta_{T E F_{\text {Left }}}-\delta_{T E F_{\text {Right }}} \\
d_{L E I}=\delta_{d L E I}=\delta_{L E I_{\text {Left }}}-\delta_{L E I_{\text {Right }}} \\
d_{L E O}=\delta_{d L E O}=\delta_{L E O_{\text {Left }}}-\delta_{L E O_{\text {Right }}}
\end{gathered}
$$

The list of model outputs is shown in table 2 with a visual schematic in figure 14:

\section{Table 2: List of flight Dynamics Outputs}

\begin{tabular}{|l|l|}
\hline Output & Description \\
\hline phi & body roll attitude angle \\
\hline NY & body lateral acceleration \\
\hline theta & body pitch attitude \\
\hline NZ & body normal acceleration \\
\hline $\mathrm{p}$ & body axis roll rate \\
\hline $\mathrm{q}$ & body axis pitch rate \\
\hline $\mathrm{r}$ & body axis yaw rate \\
\hline
\end{tabular}




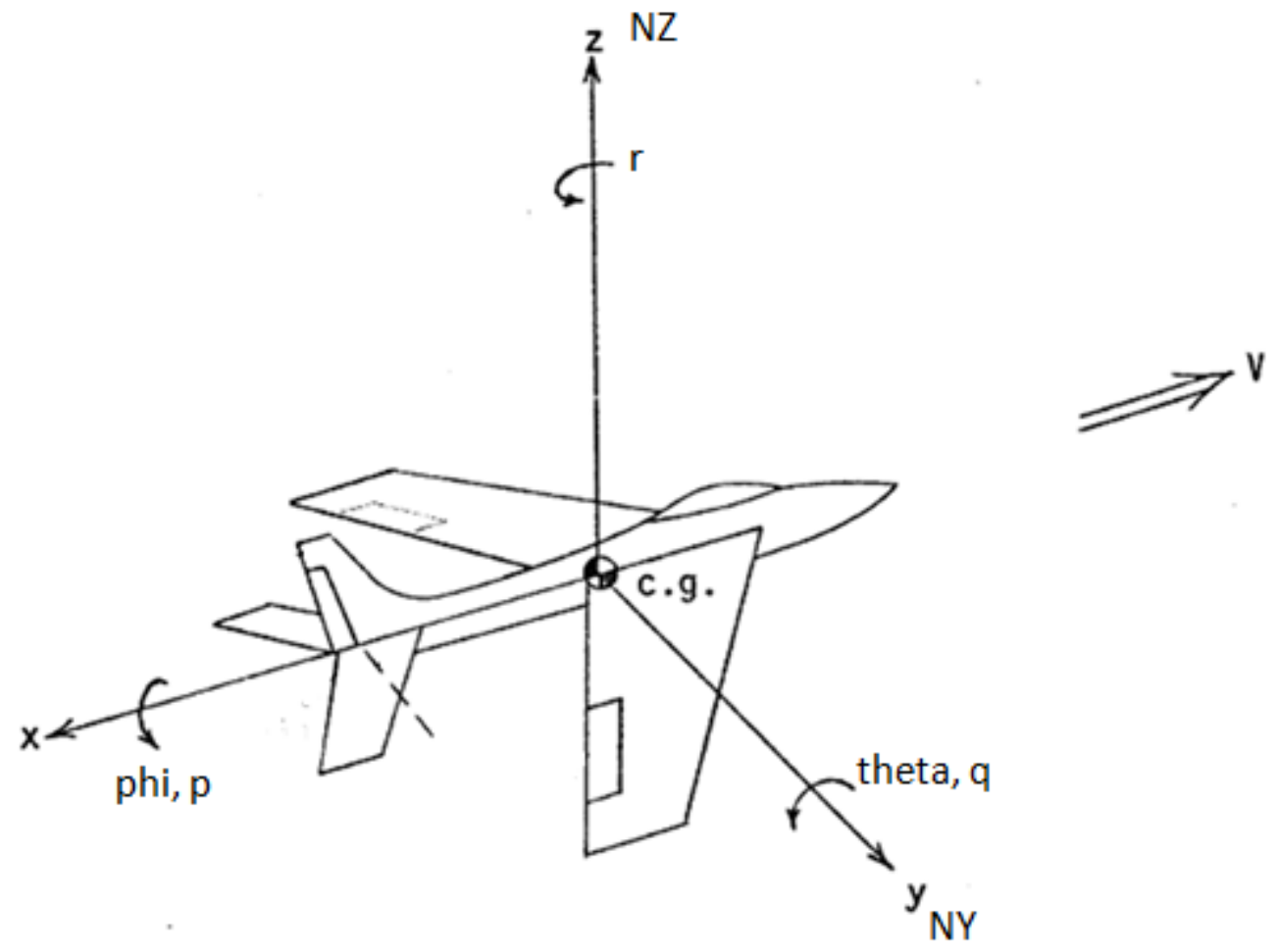

Figure 14: Flight Dynamics Schematic [10]

\subsection{Using Data from AAW to Provide Elastic Characteristics to F/A-18 Simulator}

Aircraft dynamics at specific test flight conditions were collected during the AAW flight tests. The process to convert this information into a working model that the F/A-18 simulator can use is based on a modeling process derived by Brenner, Prazenica, and Lind [11,20,21]. In order to create an accurate model from flight data that takes into account the elastic dynamics of the aircraft, a nominal model was first created as an initial estimate to represent the true aircraft. The flight data was used to generate norm-bounded operators as the errors with the nominal model. With this knowledge, flight data was incorporated into the model through an update process of the nominal model. This is in contrast to direct model identification from the flight data. This ensures that the flight dynamics are observed with multiple flight data sets. The ASE properties 
were characterized through a series of wavelet-basis signal decompositions and Volterra kernel non-linear modeling techniques.

The AAW program captured data relating the affects of F/A-18 flight dynamics from actuator commands and their resulting deflections of control surfaces. This information is directly tied to the aeroservoelastic properties of the airplane because it recorded and identified the actual flight responses of the aircraft from the combined aerodynamic, structural, and servo control forces on the aircraft.

The rigid body F/A-18 model is already very well modeled in the simulator. Incorporating the elastic structurally dynamic properties based on the flight conditions (Mach and Altitude) as it relates to the actuator inputs, allows for the modeling of the F/A-18 simulator with both the rigid and elastic properties.

The ASE calculation of the flight dynamics occurs in parallel between the original rigid body F/A-18 simulator and the ASE components of flight. Therefore interference is minimized with the F/A-18 sim which has already been proven to be accurate and robust. The ASE component can also be freely turned on and off and so that it will not affect the normal operation of the original simulator. 


\section{Chapter 4}

\section{Data Processing}

Data was received from the AAW program at 25 selected test point flight conditions. Each of these models were tested and verified for stability.

\subsection{Steady State Stability}

The ASE models received were analyzed at each flight point condition. To determine the stability of each ASE model, constant inputs were inserted into the state space models and outputs were plotted through time. The model outputs are analyzed through connecting constant inputs and plotting the outputs through the state space calculation below:

$$
\begin{aligned}
& x_{k+1}=A_{i} * x_{k}+B_{i} * u_{k} \\
& y_{n}=C_{i} * x_{k+1}+D_{i} * u_{k} \\
& u_{k}=\text { Inputs Condition Values } \\
& y_{n}=\text { Output Condition Values } \\
& x_{0}=0 \quad \text { (Initial value set to zero matrix) }
\end{aligned}
$$

The steady state stability of the flight points were verified for both ABCDX and PLANTX matrices. An example case is shown in figures 15 and 16 at Altitude $=10000 \mathrm{ft}$ and $\mathrm{Mach}=0.60$ 
Note that the y-axis units are neglected due to different corresponding flight dynamic units in this generalized plot.

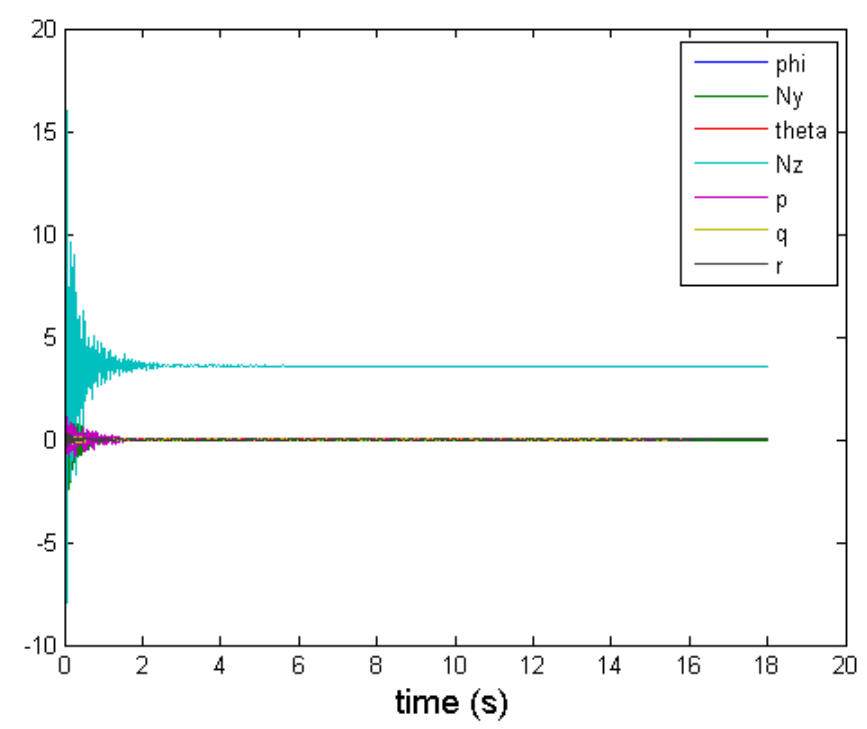

Figure 15: ABCDX steady state results

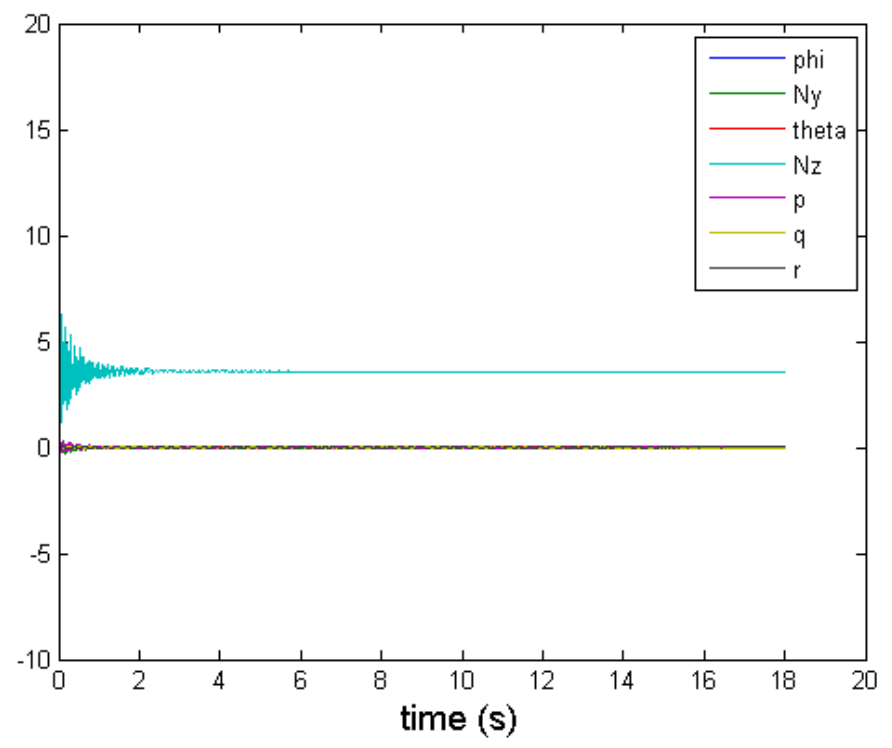

Figure 16: PLANTX steady state results 


\subsection{ASE Model Integration Flow}

The flow process to produce ASE models and integrate into the F/A-18 simulator is shown below in Figure 17.

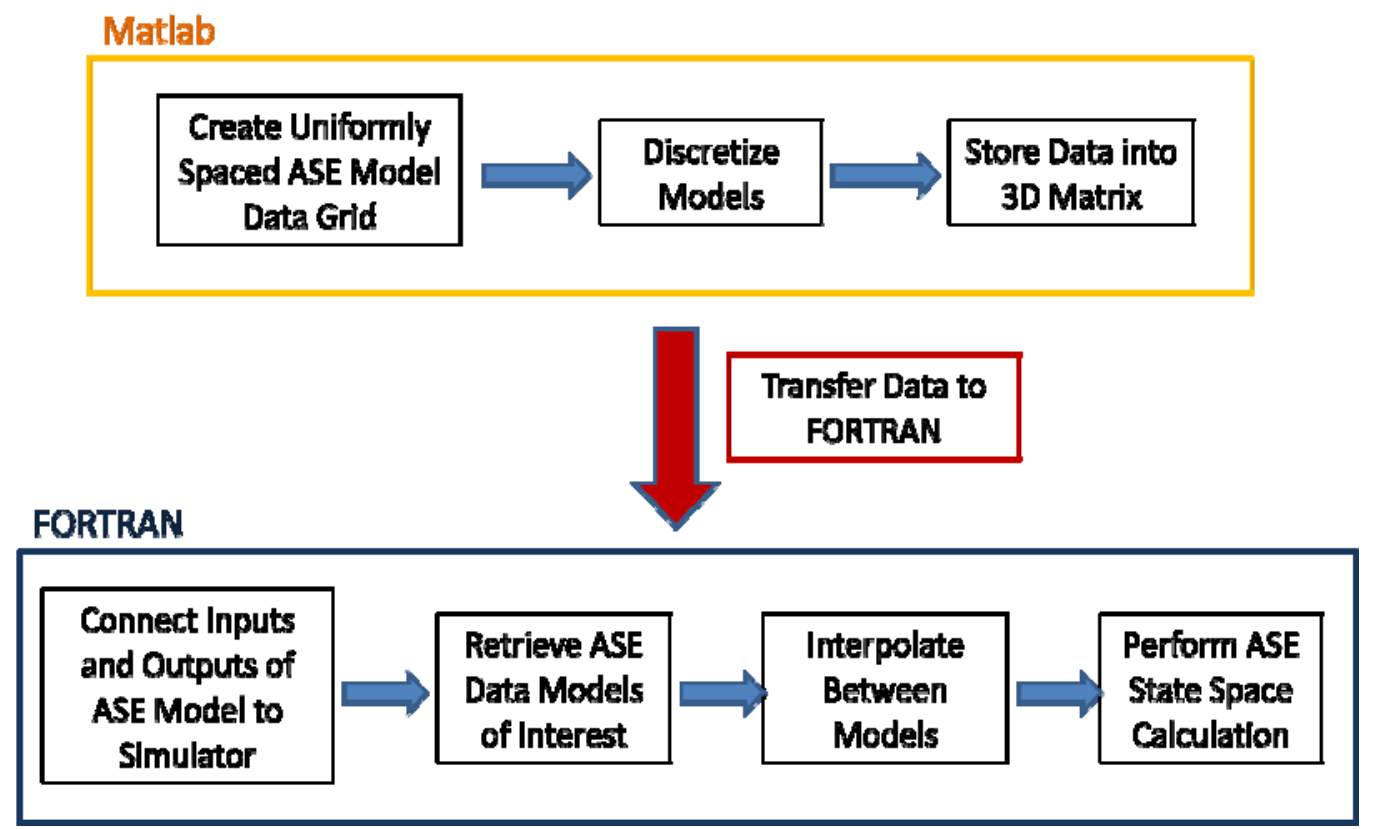

Figure 17: ASE Model Flow Process

Initial ASE models were provided as a workspace of variables in a Matlab '.mat' file. To minimize calculations in FORTRAN, the ASE models were discretized in Matlab. The discretized ASE models were then organized into a three dimensional matrix format to be transferrable for abstraction in the FORTRAN environment. Final ASE model interpolation and integration occurred in the FORTRAN simulation environment.

\subsection{Filling in Missing Flight Data Points through Linear Interpolation}

To simplify implementation of data in the F/A-18 simulator, it was necessary to create a uniformly spaced regular data base grid throughout the entire possible flight envelope of the F/A18. The data grid was broken down into altitude increments of $5000 \mathrm{ft}$ and 0.05 mach throughout 
the flight envelope of 5000-30000 ft in altitude and 0.50-1.30 in mach. This created a data grid with a total of 102 points to reference. Using linear interpolation, data matrices were determined at the unknown grid points from known flight data. The step by step process for creating the baseline data base is shown below:

1) First perform all direct linear interpolations possible

2) Copy data where no bounded interpolation is possible

3) Interpolate newly bounded points as appropriate

4) Interpolate all remaining points with respect to mach

The linear interpolation used the standard linear interpolation formula:

$$
y=y_{a}+\left(y_{b}-y_{a}\right)\left(\frac{x-x_{a}}{x_{b}-x_{a}}\right)
$$

where $x$ represents the data location and $y$ represents the data location.

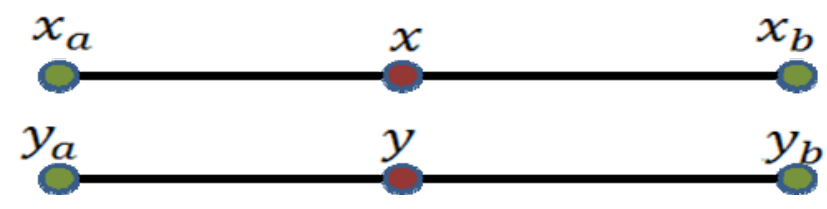

\section{Figure 18: Linear Interpolation Visualization}

First, this equation was used to interpolate between two known flight conditions for conditions where altitude was constant. Figure 19 shows the initial flight data set that was received. 


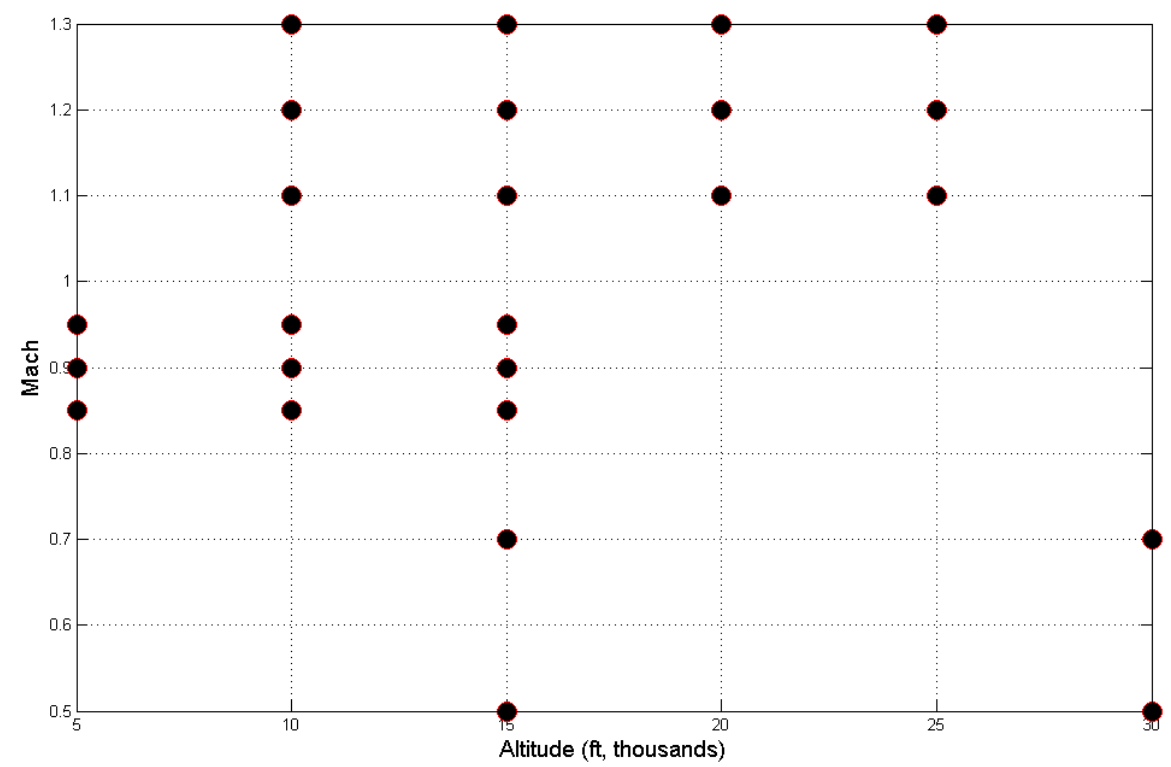

Figure 19: Initial Data Set

Figure 20 shows the updated data set where data was linearly interpolated between known flight condition models.

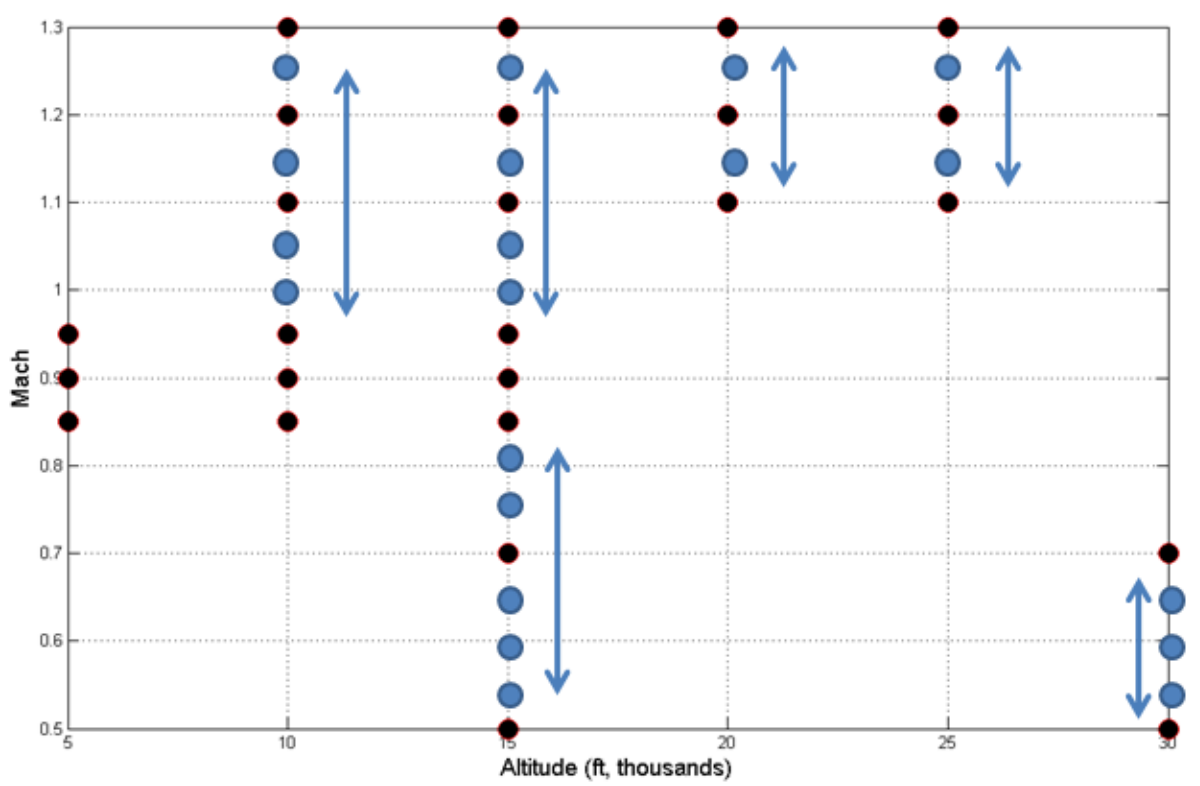

Figure 20: Linearly Interpolated Flight Condition Models

Second, for points where no bounded interpolation is possible, data is copied based on flight data or interpolated data with respect to constant mach as shown in Figure 21. 


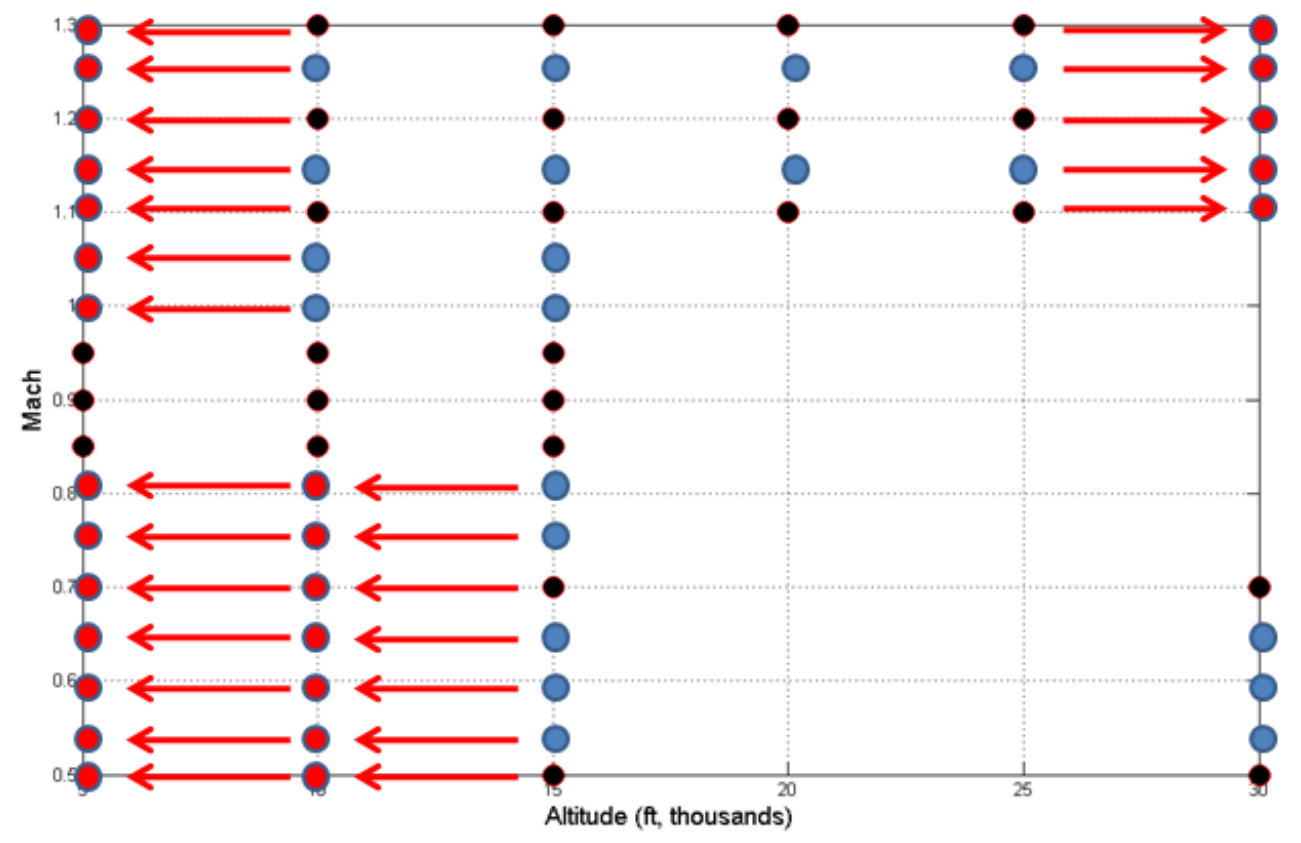

Figure 21: Copied ASE Models With Respect to Constant Mach

Third, with new copied data points, interpolation between two known flight conditions continued for conditions where altitude was constant as shown in Figure 22.

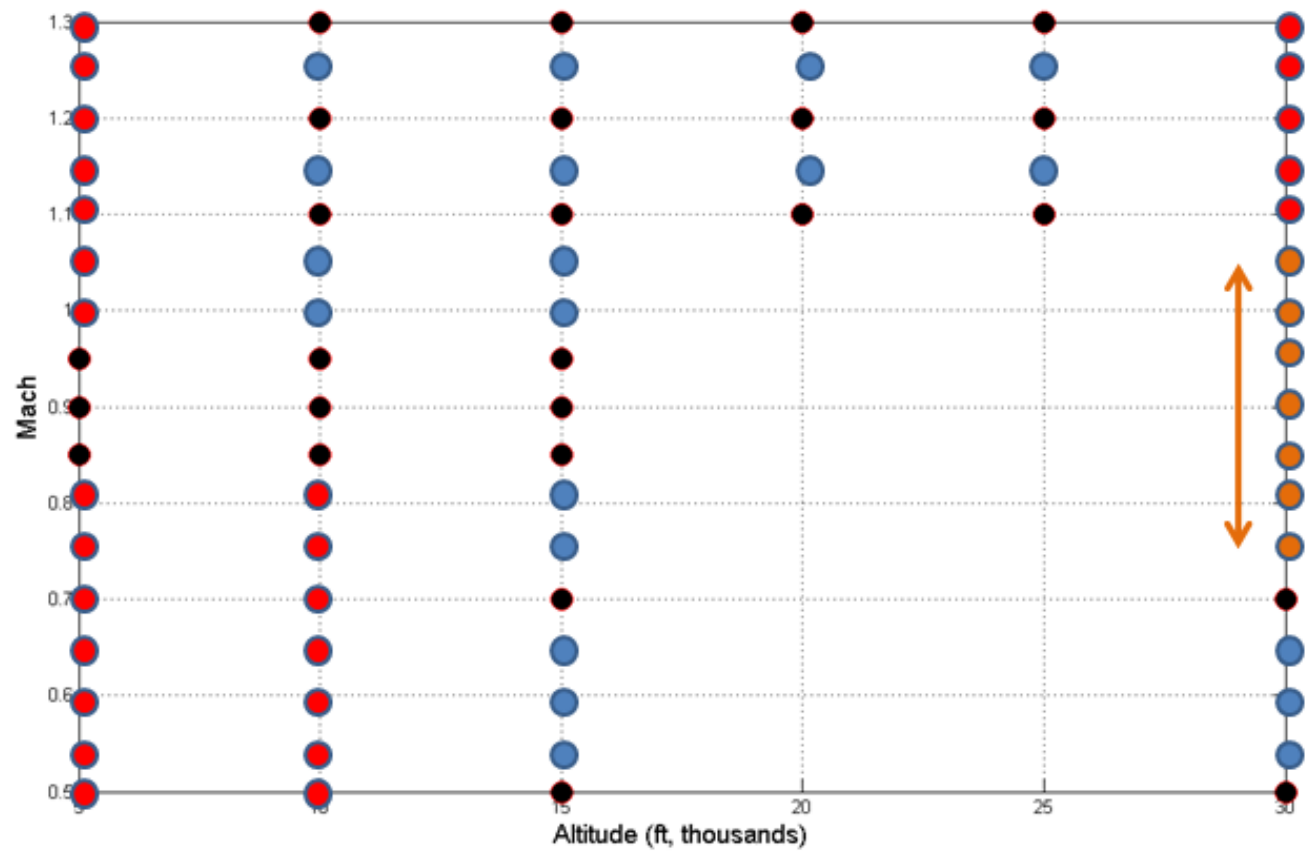

Figure 22: Linearly Interpolated Models at 30K Flight Condition 
Fourth, points that are not in between known flight data will reference interpolated values and linearly interpolate with respect to a constant mach as shown in Figure 23.

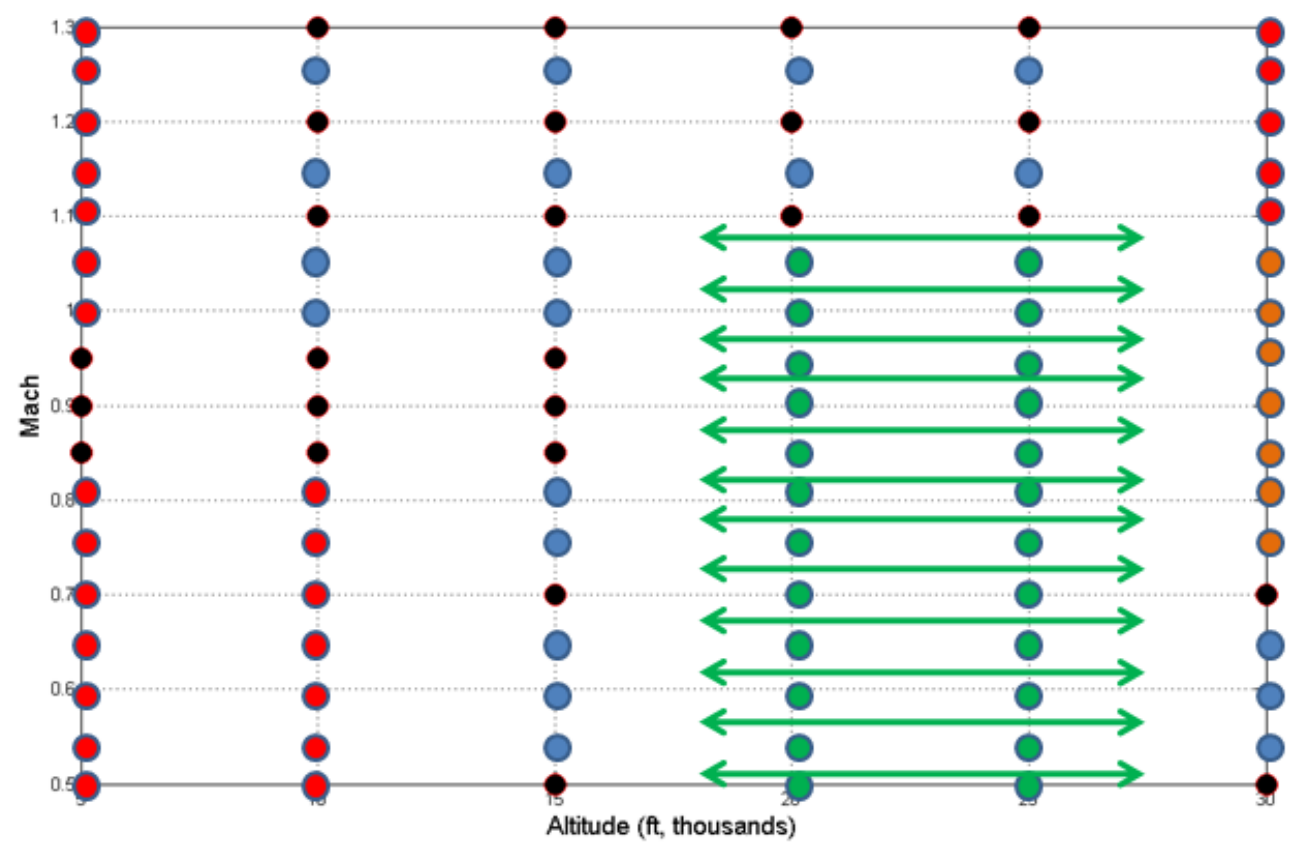

Figure 23: Linearly Interpolated ASE Models With Respect to Constant Mach

Finally, the entire baseline data set is established as shown in Figure 24.

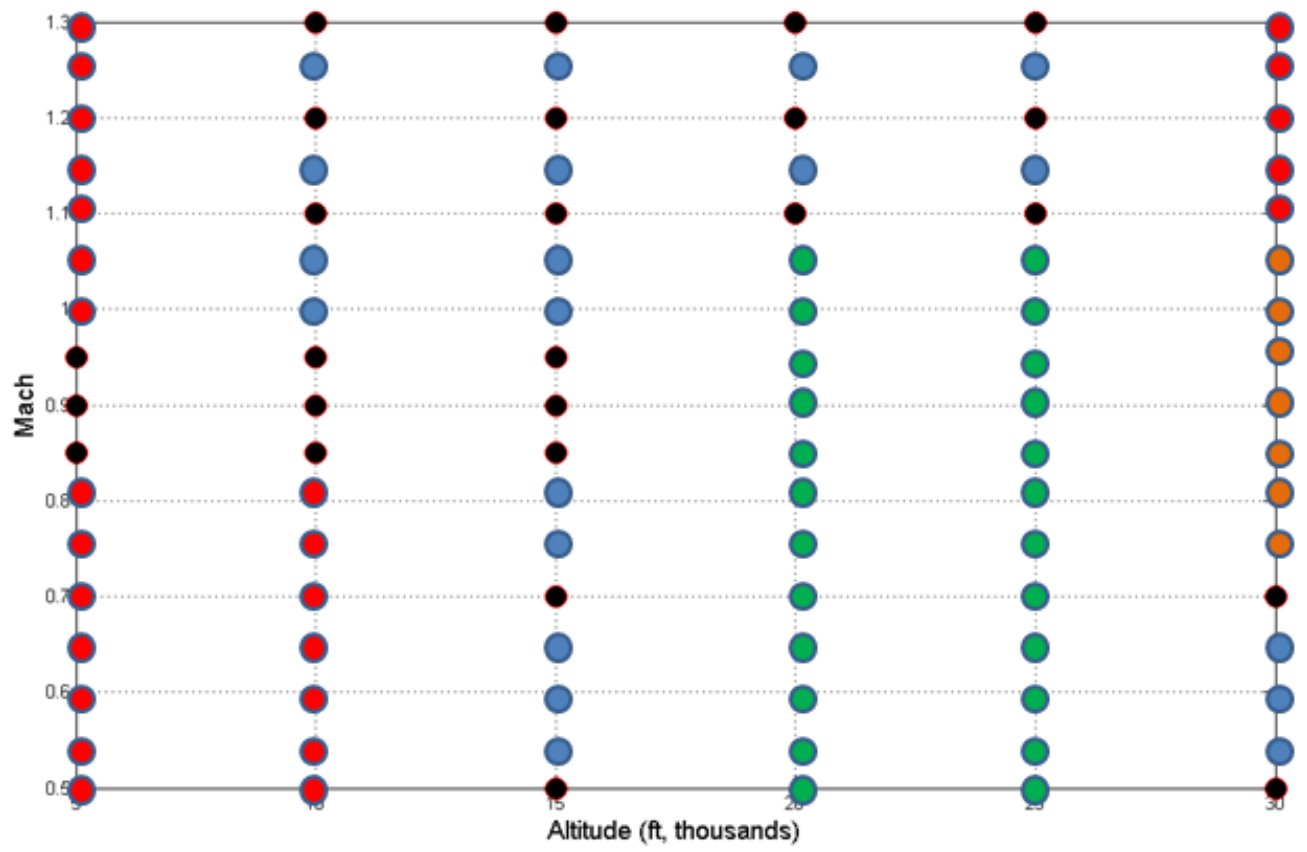

Figure 24: Complete ASE Baseline Model Set 
Note that extrapolation of data is not feasible as this no longer guarantees stability of matrices.

A representative unit-less matrix element is used to visually show the results of the interpolation process throughout the flight envelope as shown in Figure 25.

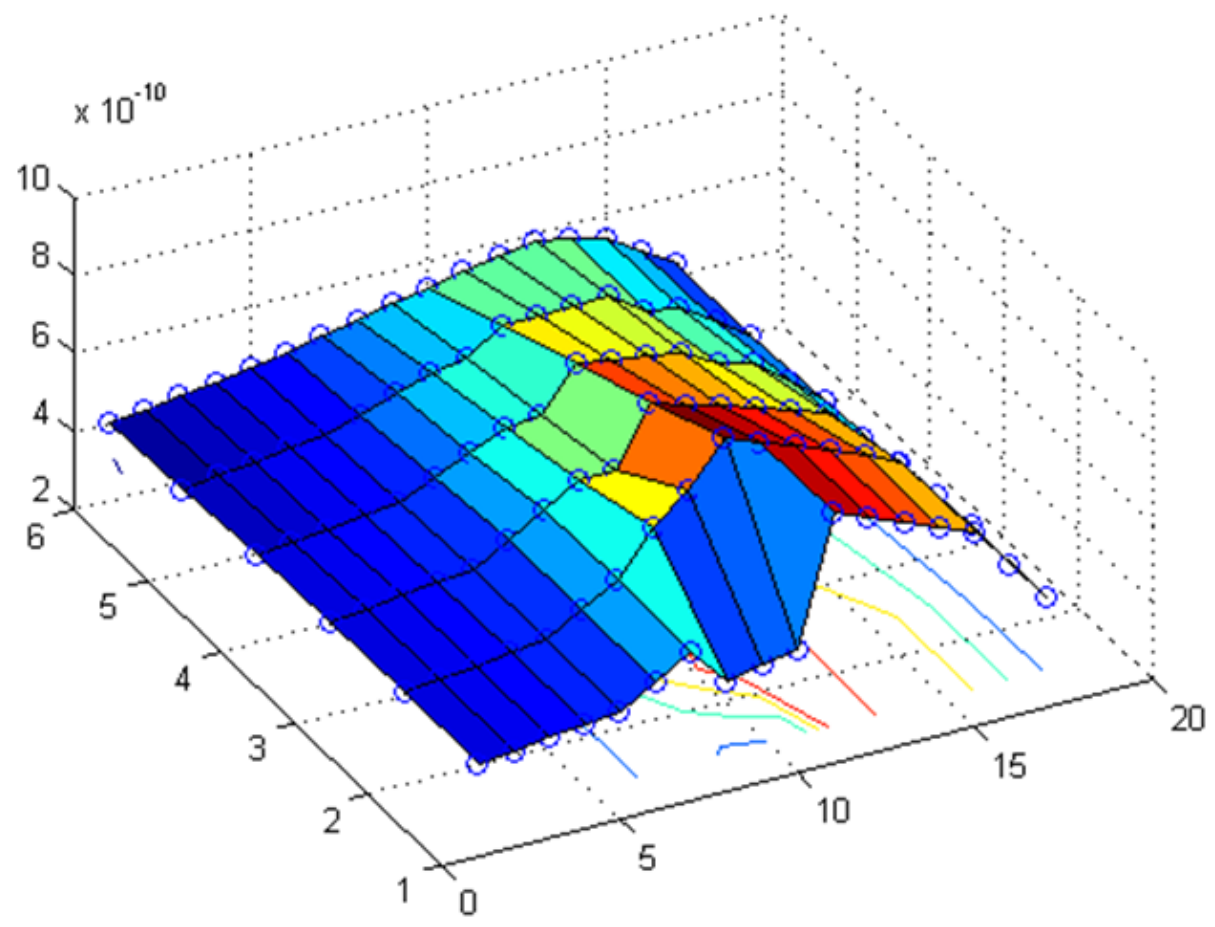

Figure 25: Representative Matrix Element of Baseline Matrix Plotted Throughout Entire Flight Envelope

\subsection{Data Organization and Storage}

To simplify data storage and transfer into the F/A-18 simulator, specifically so that it can be read in FORTRAN, the data for all of the flight conditions were organized into a three dimensional matrix. Each of the state space matrices: A, B, C, and D would have its own '.DAT' data file for transfer into the FORTRAN programming environment. The third dimension shall be called the 'depth' of the matrix; therefore the depth of the matrices will be 102 , since this is the total number of data conditions. The storage of the data was organized with respect to mach as the major variable and altitude as the minor variable. This means that each 
depth will run through all altitudes for a given mach condition before moving onto the next mach condition. For example, a depth of 1 references data at Mach $=0.50$ and Altitude $=5000 \mathrm{ft}$. A depth of 2 references data at Mach $=0.50$ and Altitude $=10000 \mathrm{ft}$. A depth of 7 references data at Mach $=0.55$ and Altitude $=5000 \mathrm{ft}$. Finishing off at a depth of 102 where Mach=1.30 and Altitude $=30000 \mathrm{ft}$. Figure 26 demonstrates this concept.
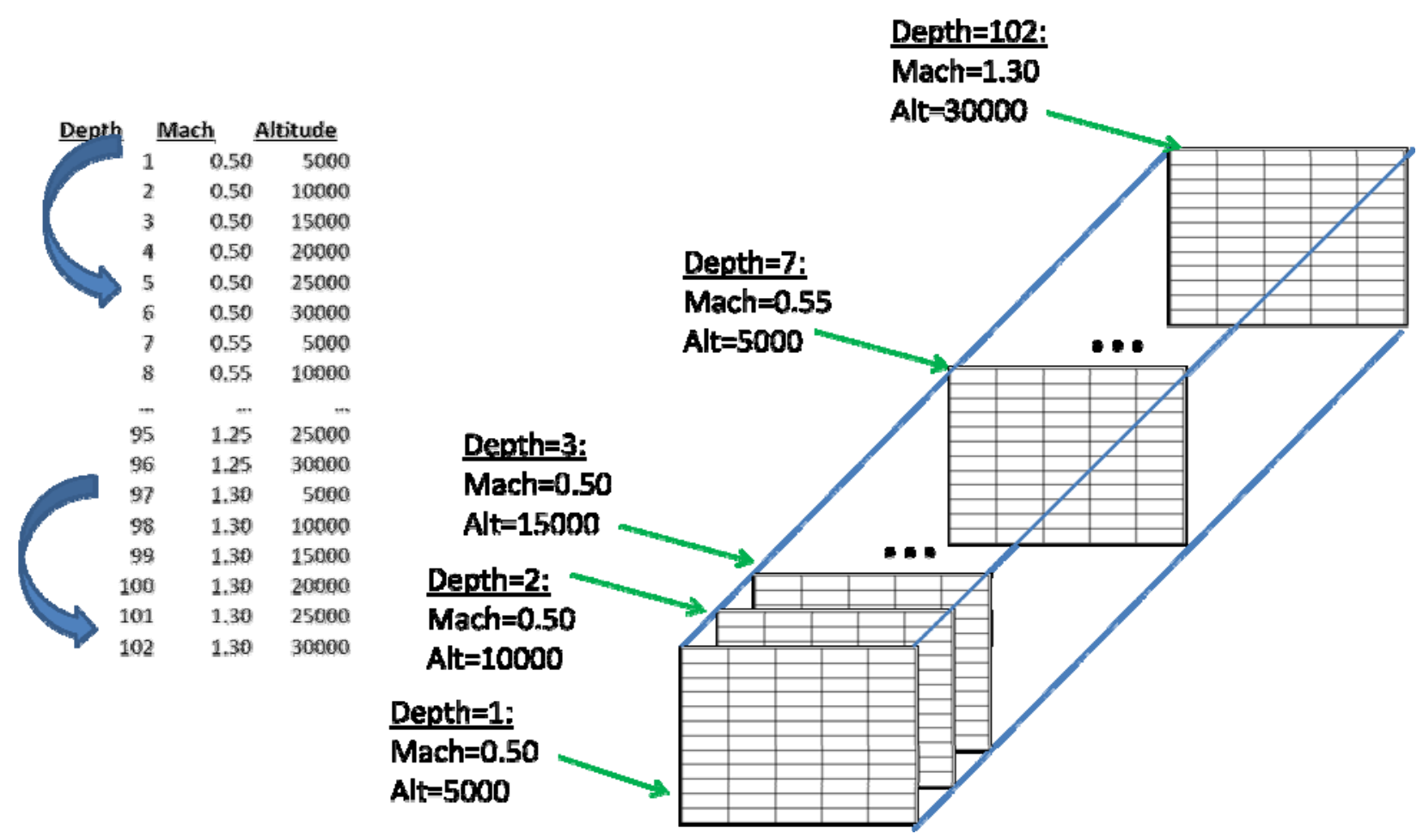

Figure 26: Storage of ASE Models into 3D Matrix

Notice that altitude will reset after every 6 depth terms since that is the total number of altitude possibilities.

\subsection{Discretization of Data}

The F/A-18 simulator performs all calculations in discrete steps at a rate of $160 \mathrm{~Hz}$ (160 steps per second). Therefore it is necessary that the incoming state space models, specifically their A, B, C, and D matrices are appropriately discretized. Since the ASE flight dynamics have 
characteristics that can only be realized at a higher frequency, the ASE data were discretized at a higher rate. Both $\mathrm{ABCDX}$ and PLANT matrices were discretized at twice the run rate of the sim, at $320 \mathrm{~Hz}$.

The discretization was performed using Matlab's 'c2dm' command. The 'c2dm' command is the same as the 'c2d' command, but reads in the A, B, C, D matrices directly instead of expecting a Linear Time Invariant (LTI) model format.

The Matlab discretization command required the discretization rate and method for discretizing. The rate will be $320 \mathrm{~Hz}$ (1/320 seconds) and the method used will be the zero-order hold.

The zero-order hold takes a sequence of samples and extrapolates or holds them to produce a continuous signal. Suppose we have the function, e $(\mathrm{t})$, as sketched in Figure 27. The zero order hold operation uses a zero-order polynomial(constant) to approximate e(t). Therefore, pulling data at discrete points will characterize the curve. The higher the frequency, the more accurate the curve is characterized using a zero order hold. Using the zero-order hold method assumes that the control inputs are piecewise constant over the sampling period (1/320 s) [23].

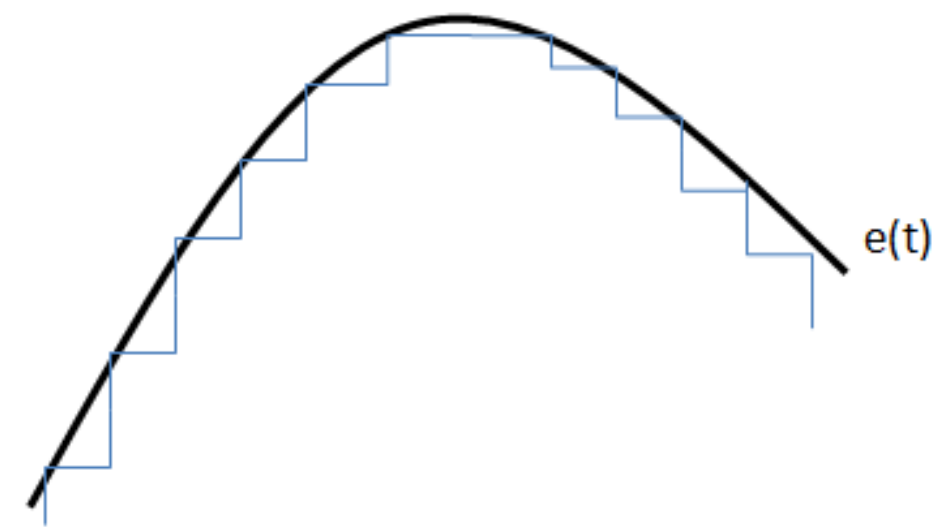

Figure 27: Zero Order Hold Concept Therefore, discretizing at $320 \mathrm{~Hz}$ shows a breakdown 
Figure 28 illustrates this concept for the F/A-18 simulator where the black line is the continuous model, the blue line is the discretized model at160 Hz (sim time), and the green line is the discretized model at $320 \mathrm{~Hz}$. The higher frequency is able to capture more of the higher order dynamics in the discretization process.

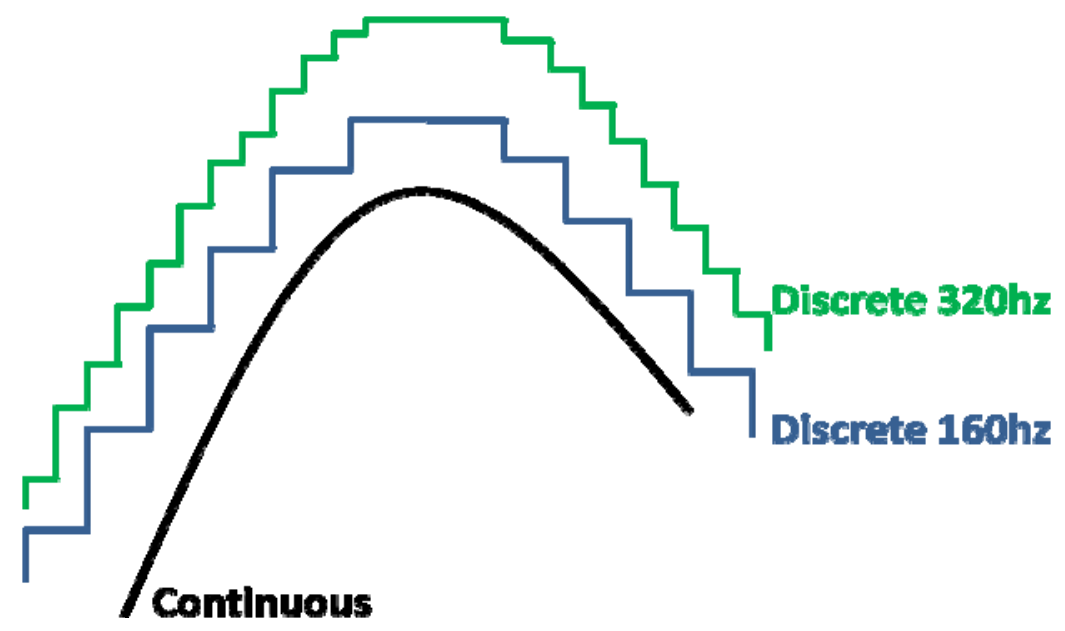

Figure 28: Visual Schematic of Discretization of Data

Matlab's simple zero order hold discretization process uses the following method [23,24,32]:

Converts Continuous time model:

$\dot{x}=A x+B u$

Into Discrete Model:

$x_{n+1}=\Phi x_{n}+\Gamma u_{n}$

Where

$\Phi=e^{\bar{A} T}$

$\Gamma=\int_{\lambda}^{T} e^{\bar{A} t} \bar{B} d t$

$y=C x+D u$ 


\section{Chapter 5}

\section{ASE Integration into Sim}

\subsection{Data Retrieval and Interpolation}

FORTRAN reads in '.DAT' data files as a database reference. To ensure the sim would not need to load the data set for every calculation, a separate initialization file is written to only load the data upon start up of the sim.

Since data is organized as a 3D matrix, each depth is unique to each flight condition. Based on the current flight condition (altitude and mach), the script recognizes which four flight conditions surround the desired data point to be used for interpolation. Additionally, the code is written to ensure flight conditions outside the data bounds can still be characterized by at least referencing the nearest flight condition. Also, if the flight condition is at an actual data point, the interpolation will reduce to only provide the ASE model at the data point and no interpolation is required. The same logic of interpolation reduction would also occur if the flight condition is currently at a point exactly aligned with the mach or altitude of the data point. The four depth values retrieve data in reverse at which they were stored as shown in Figure 29: 


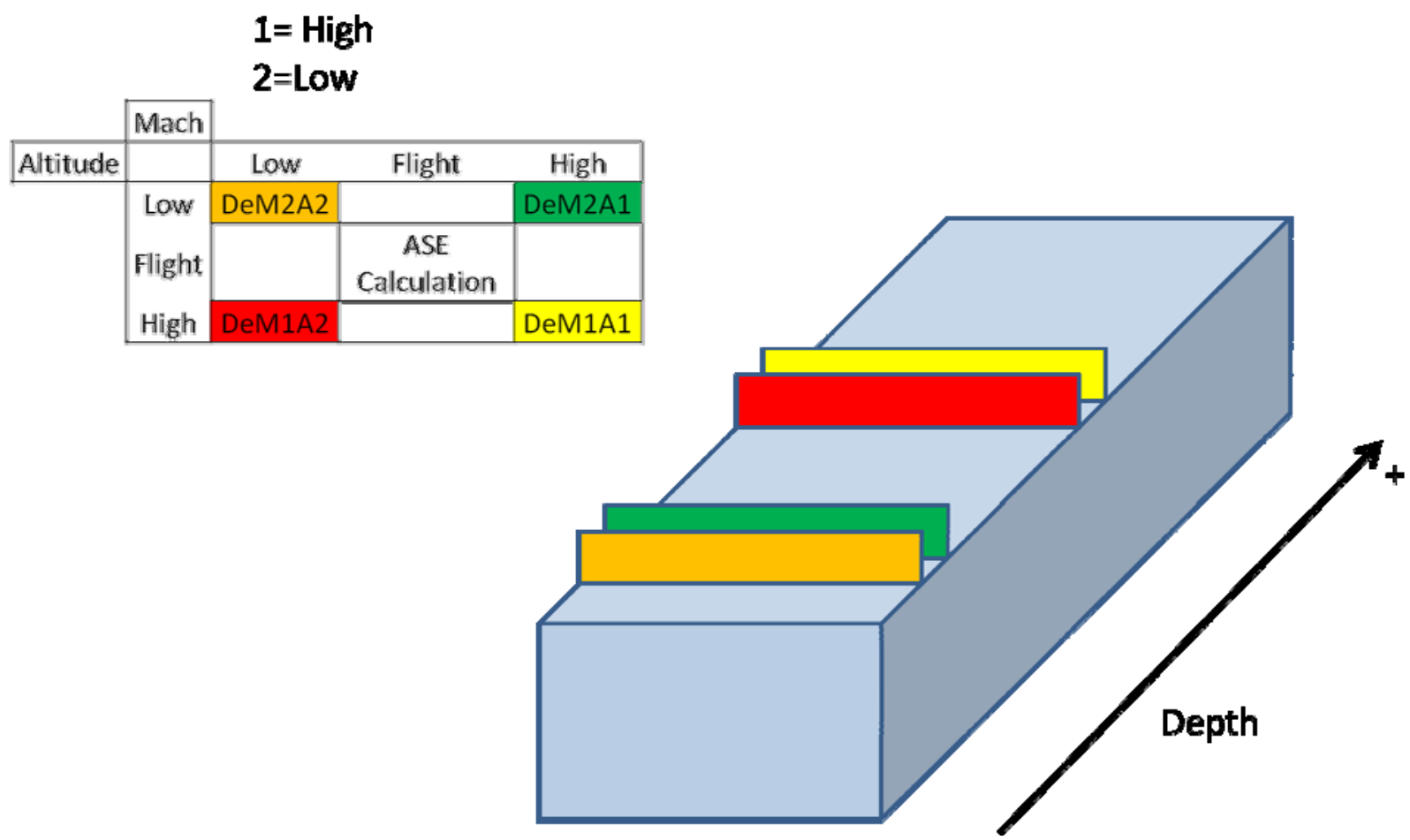

Figure 29: Data File Retrieval Diagram

The interpolation then proceeds via a Double Linear Triangular Interpolation method which is derived and shown in Appendix A.

This method was chosen over normal triangular interpolation, because triangular interpolation assumes the data is completely planar. In fact, the four points do not lie on the same linear plane and data must be correctly interpolated accordingly. Additionally, bilinear interpolation was not used because it uses a quadratic interpolation scheme that had the potential to inaccurately produce models outside of the exact linear stability criteria.

\subsection{ASE Conversion}

The ASE state space model takes 11 inputs and yield 7 outputs. Since the F/A-18 simulator runs in discrete steps, the ASE data was discretized to integrate into the simulator. However, in order to characterize the higher order dynamics, the ASE data was discretized at twice the step rate of 
the sim. This was accounted for by doubling the number of each state space calculation before adding in the outputs to the rest of the sim.

$$
\begin{aligned}
& x_{k+1}=A_{i} * x_{k}+B_{i} * u_{k} \\
& x_{k+2}=A_{i} * x_{k+1}+B_{i} * u_{k} \\
& y_{n}=C_{i} * x_{k+2}+D_{i} * u_{k}
\end{aligned}
$$

Therefore, $x_{k}$ went through two iterations before integrating into the sim. This ensured that the ASE calculations were run at twice the rate as the rest of the sim.

\subsection{Adding into the Sim}

The outputs from the elastic model can now be added to the outputs form the rigid body model calculations. The sum of the outputs will yield the total flight dynamics of the aircraft. This information would provide net flight dynamics output into the simulator and also feed back into the control system to update the model inputs. 


\section{Chapter 6}

\section{Results}

The F/A-18 Simulation computed flights using different scenarios with the ASE models ABCDX and PLANTX integrated into the system. To test the robustness of the integrated elastic models into the simulator, conditions were tested for scenarios with no pilot inputs, and for conditions where the pilot inserted an up and down pitch doublet. Both of these scenarios were tested for the ABCDX and PLANTX models.

\subsection{Model ABCDX- 30 Seconds- No Pilot Inputs}

Figures 30-39 correspond to using elastic model ABCDX for 30 seconds of flight data allowing the simulation to drift with no pilot inputs. Figure 30 show the changing altitude and mach flight conditions through time. 

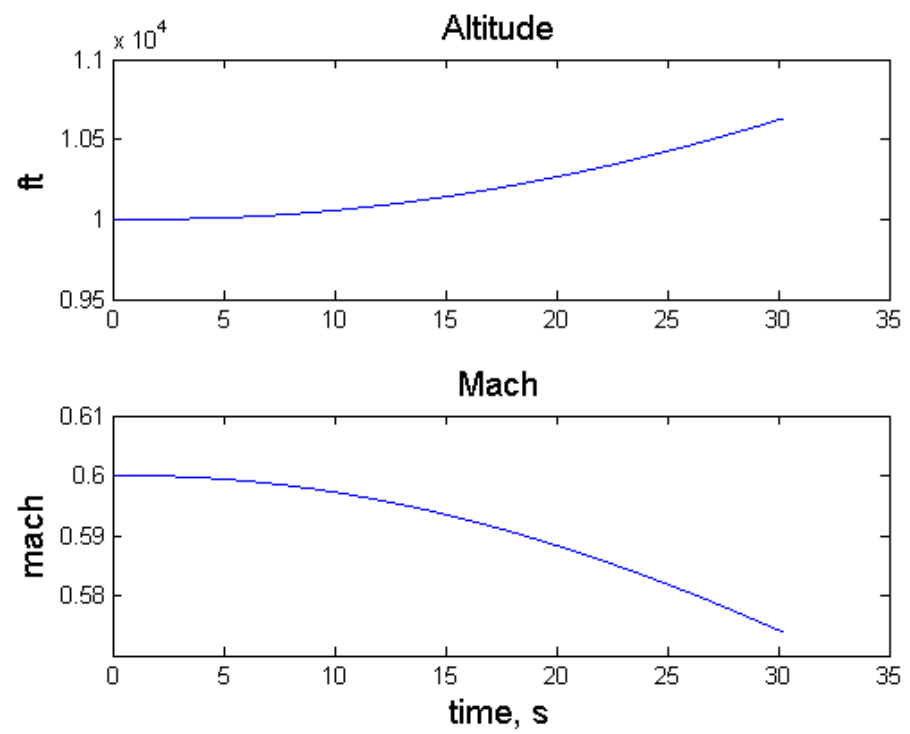

Figure 30: ABCDX Sim Steady State Plots- Flight Conditions

Figure 31 shows the rigid body contribution to the changing roll, pitch, and yaw rates through time.
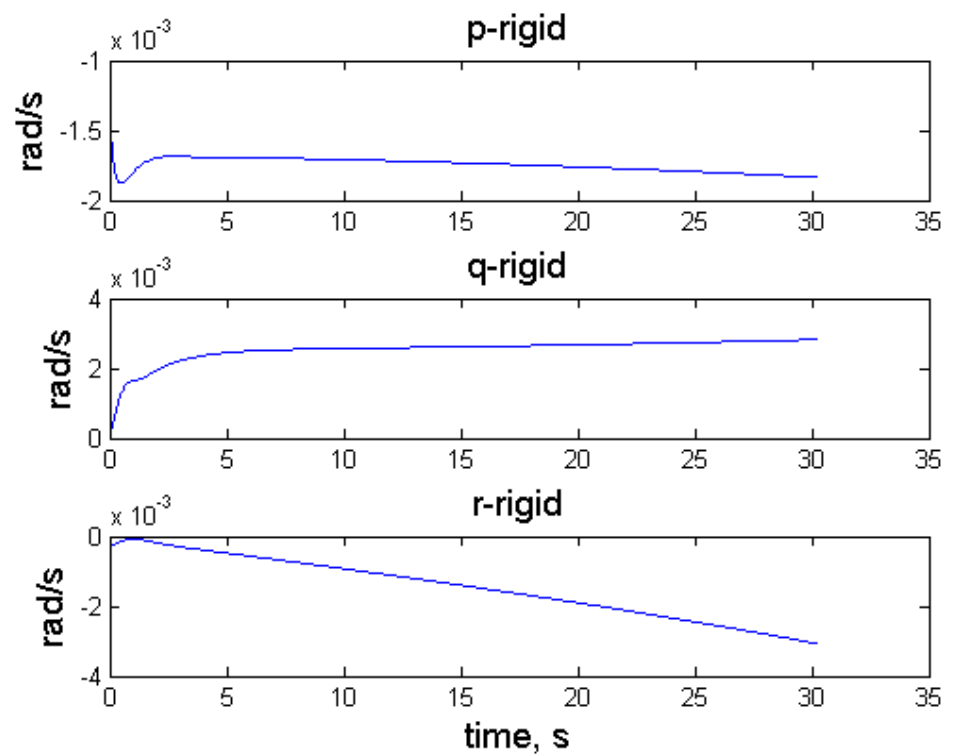

Figure 31: ABCDX Sim Steady State Plots- Rigid-Roll, Pitch, and Yaw Rate 
Figure 32 shows the elastic body contribution to the changing roll, pitch, and yaw rates through time.
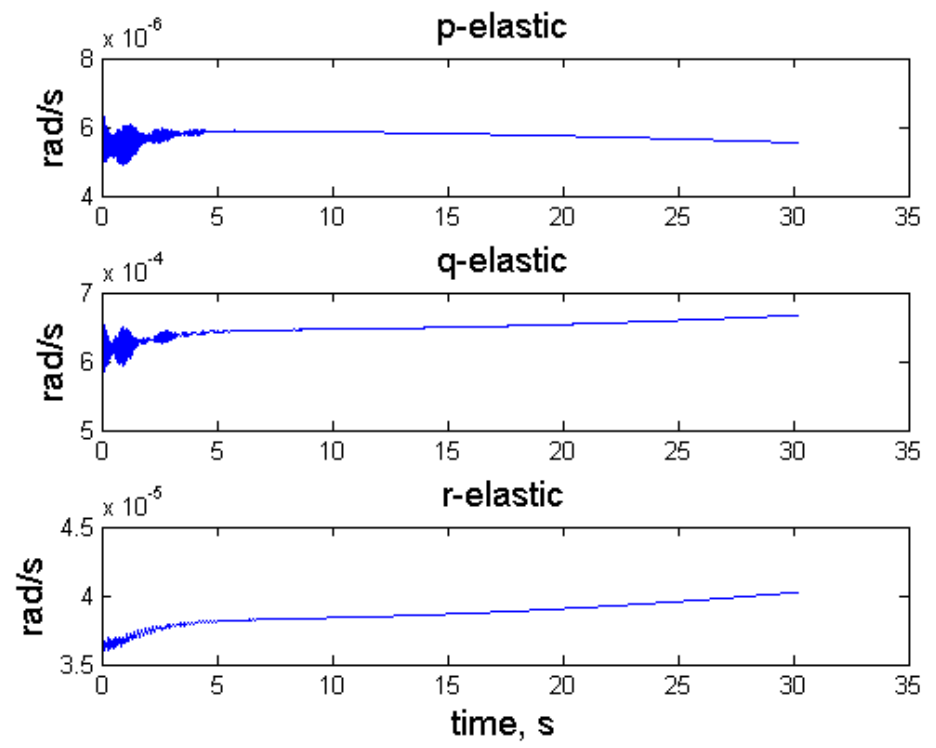

Figure 32: ABCDX Sim Steady State Plots- Elastic Component-Roll, Pitch, and Yaw Rates Figure 33 shows the percent impact of the elastic roll, pitch, and yaw rate dynamics on the total roll rate the dynamics of the aircraft.
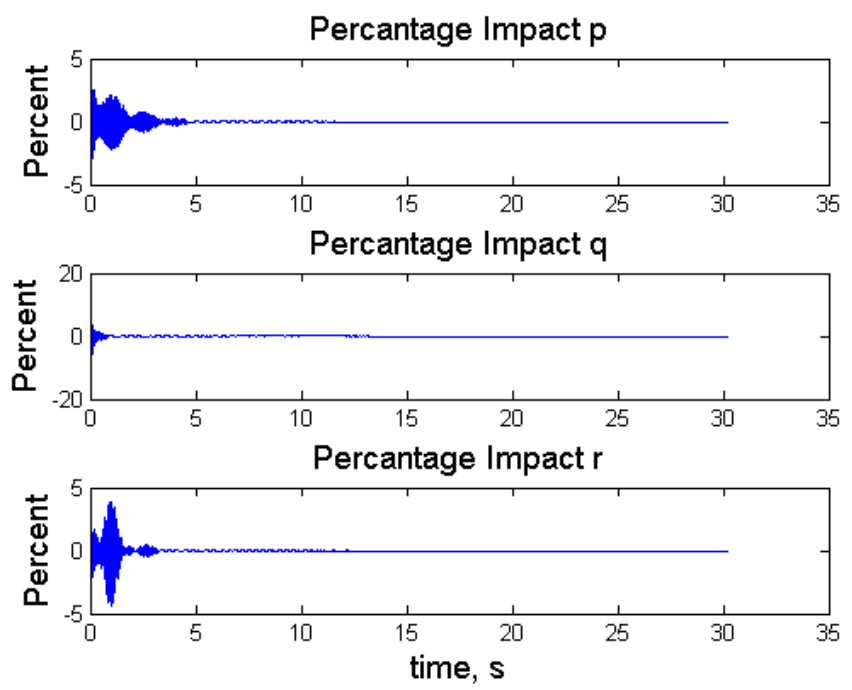

Figure 33: ABCDX Sim Steady State Plots- Elastic Component-Roll, Pitch and Yaw RatesPercentage Impact

Figure 34 shows the rigid body contribution to the changing accelerations through time. 

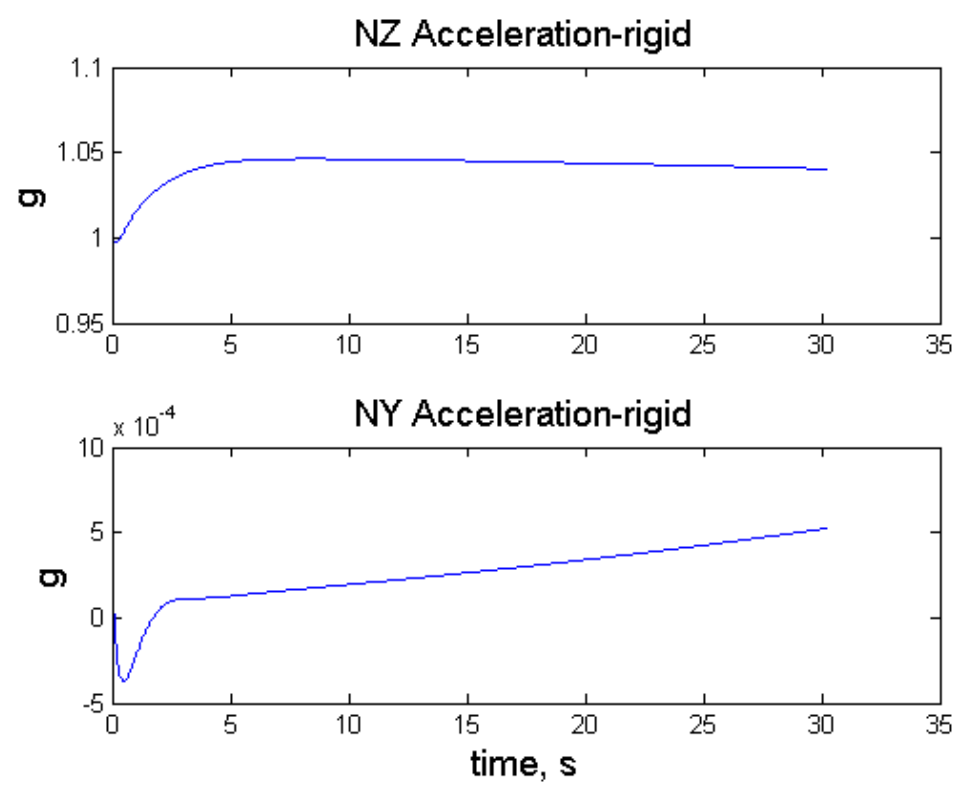

Figure 34: ABCDX Sim Steady State Plots- Rigid -Accelerations

Figure 35 shows the elastic body contribution to the changing accelerations through time.
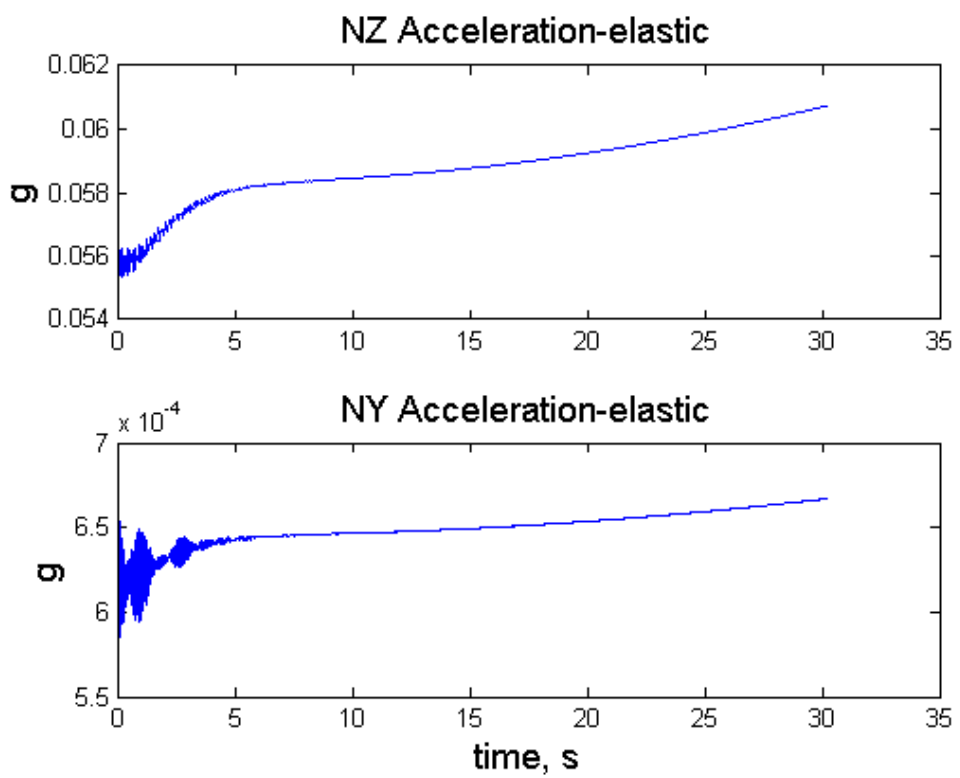

Figure 35: ABCDX Sim Steady State Plots- Elastic Component- Accelerations 
Figure 36 shows the percent impact of the elastic acceleration dynamics to the total acceleration dynamics of the aircraft.
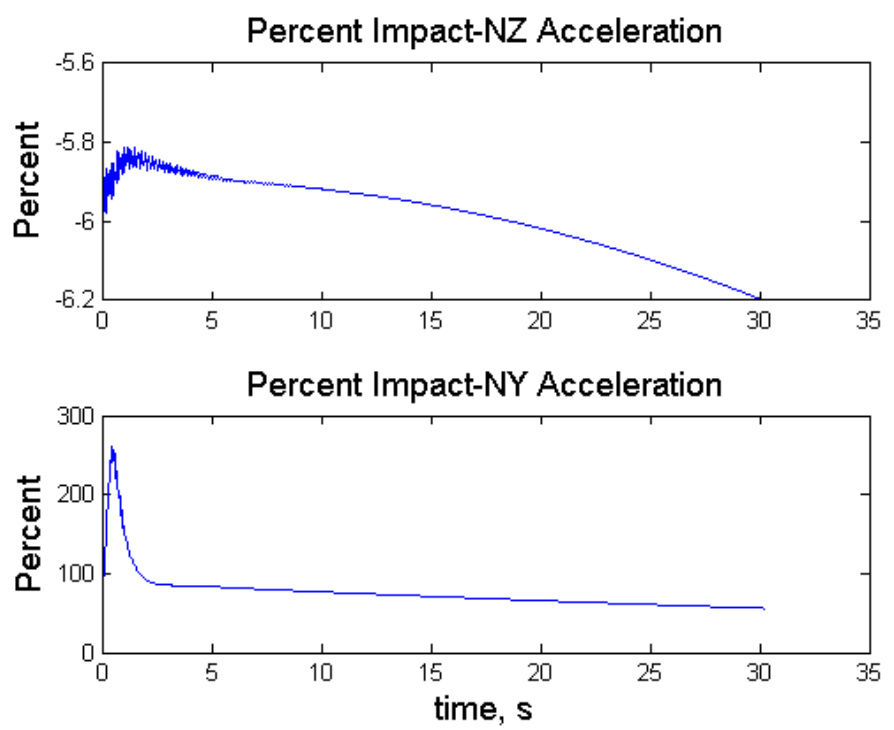

Figure 36: ABCDX Sim Steady State Plots- Elastic Component- Accelerations- Percentage

Figure 37 shows the rigid body contribution to the changing roll and pitch angle through time.
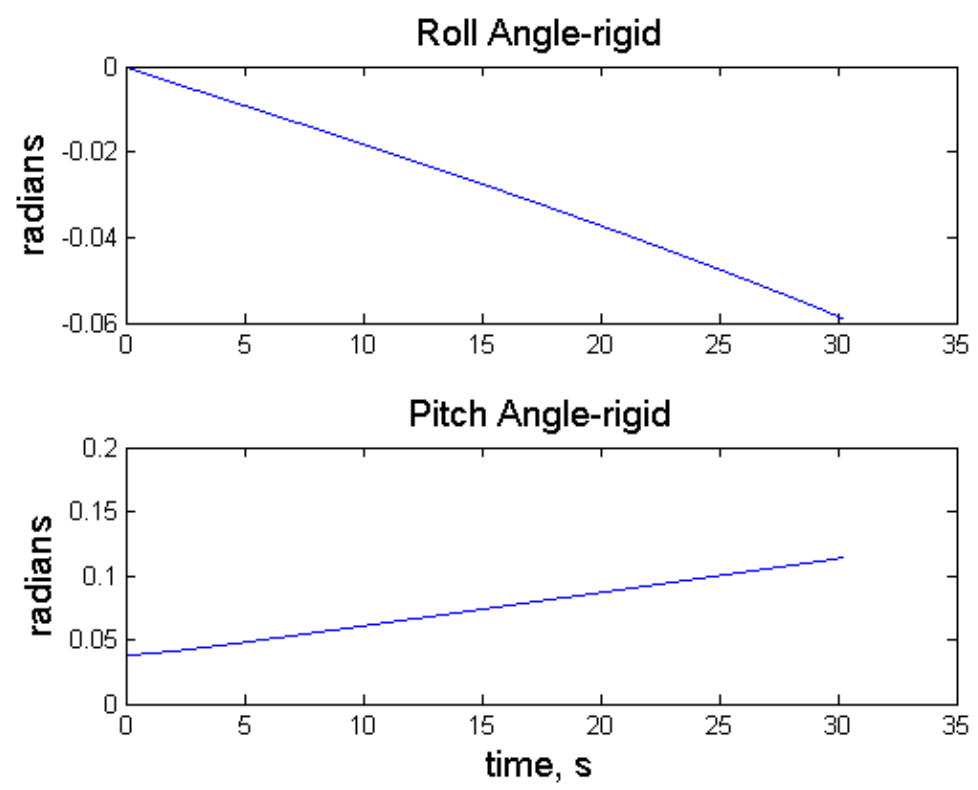

Figure 37: ABCDX Sim Steady State Plots- Rigid - Roll and Pitch Angle 
Figure 38 shows the elastic body contribution to the changing roll and pitch angle through time.
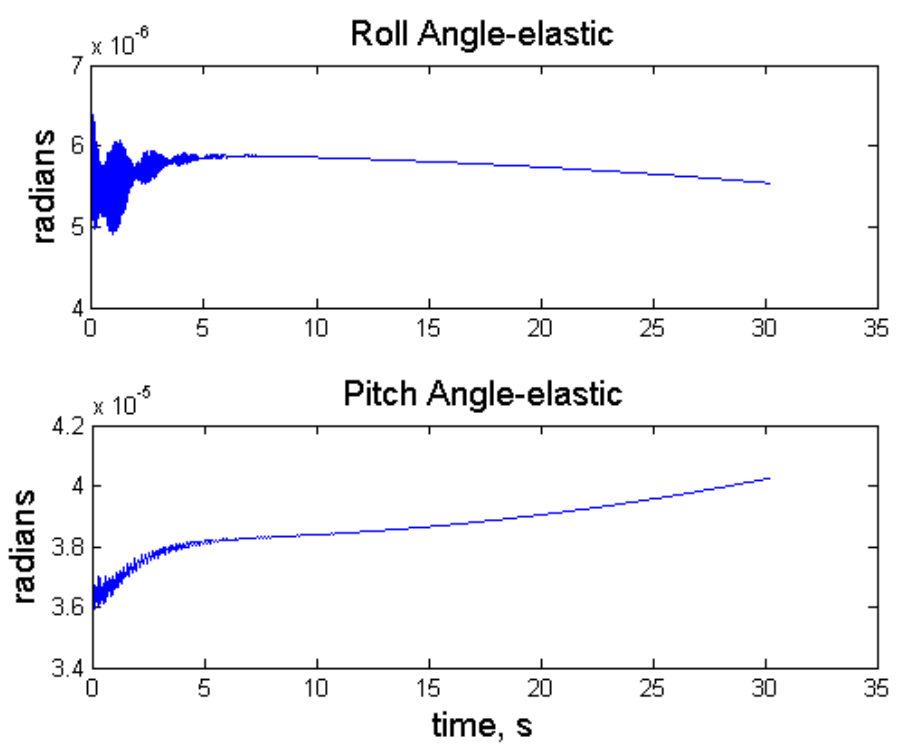

Figure 38: ABCDX Sim Steady State Plots- Elastic Component- Roll and Pitch Angles

Figure 39 shows the percent impact of the elastic roll and pitch angle contribution to the total roll angle dynamics of the aircraft.
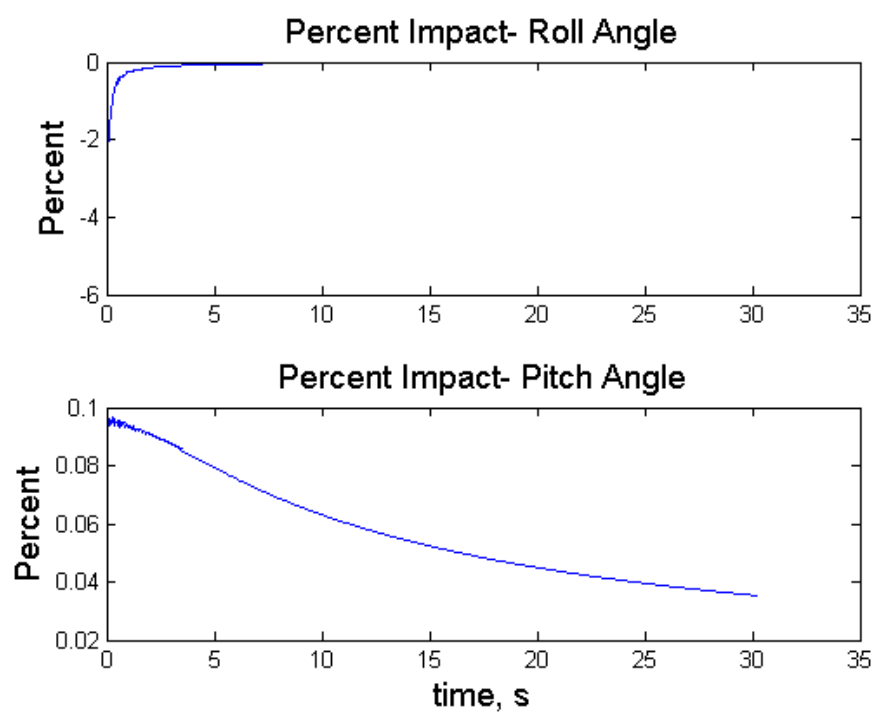

Figure 39: ABCDX Sim Steady State Plots- Elastic Component- Roll and Pitch AnglesPercentage 


\subsection{Model ABCDX- 30 Seconds- Doublet Input}

Figures 40-49 correspond to using elastic model $\mathrm{ABCDX}$ for 30 seconds of flight data allowing the simulation to drift with a pitch doublet pilot input approximately 12 seconds into the simulation. Figure 40 shows the changing altitude and mach flight conditions through time.
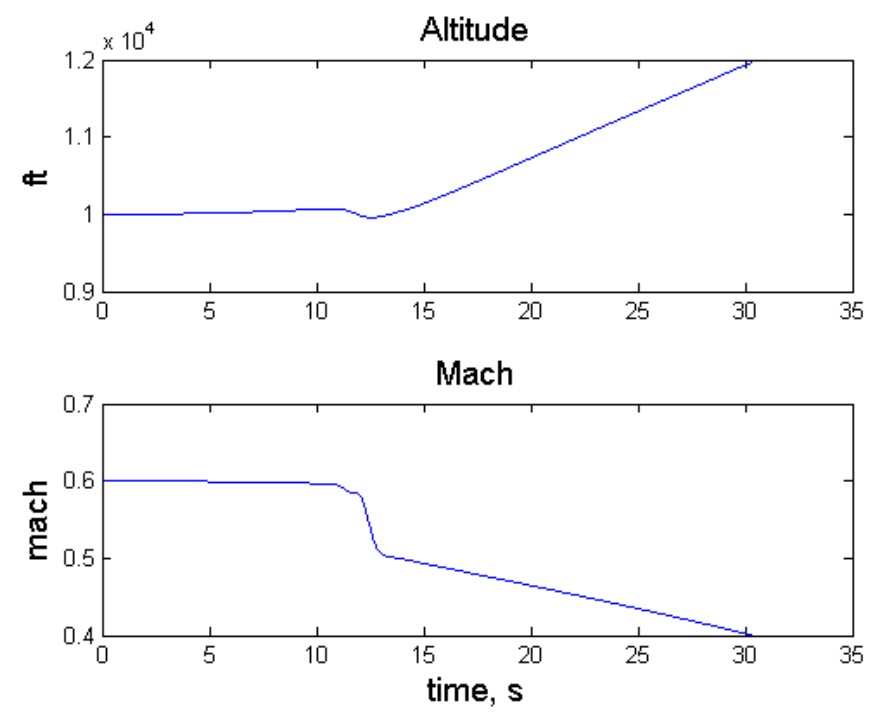

Figure 40: ABCDX Sim Steady State Plots -With Stick Doublet - Flight Conditions

Figure 41 shows the rigid body contribution to the changing rates through time. 

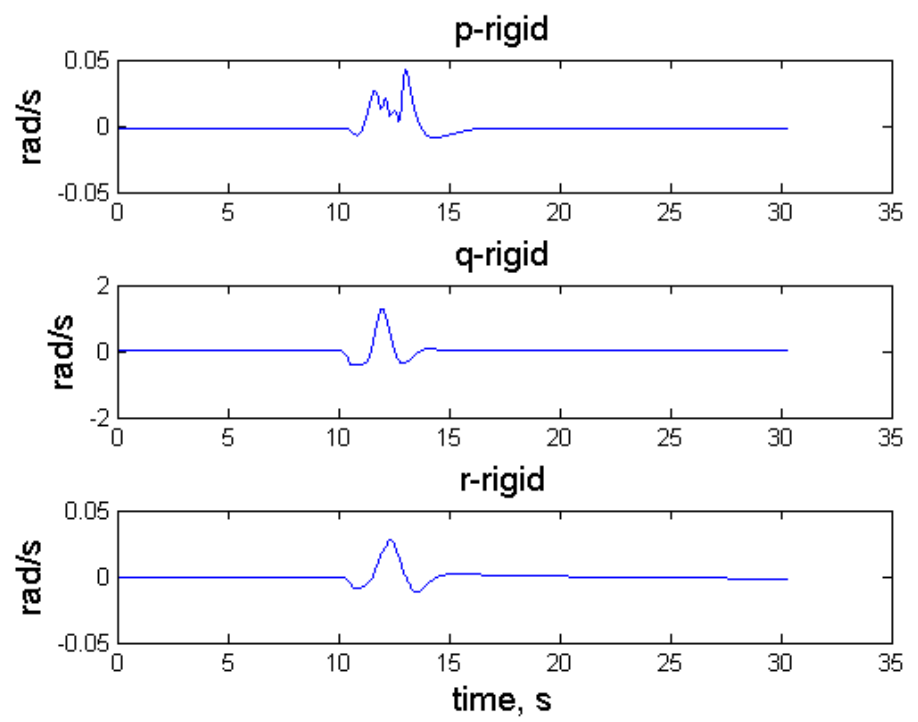

Figure 41: ABCDX Sim Steady State Plots- Rigid- Stick Doublet -Roll, Pitch, and Yaw Rate

Figure 42 shows the elastic body contribution to the changing roll, pitch, and yaw rates through time.
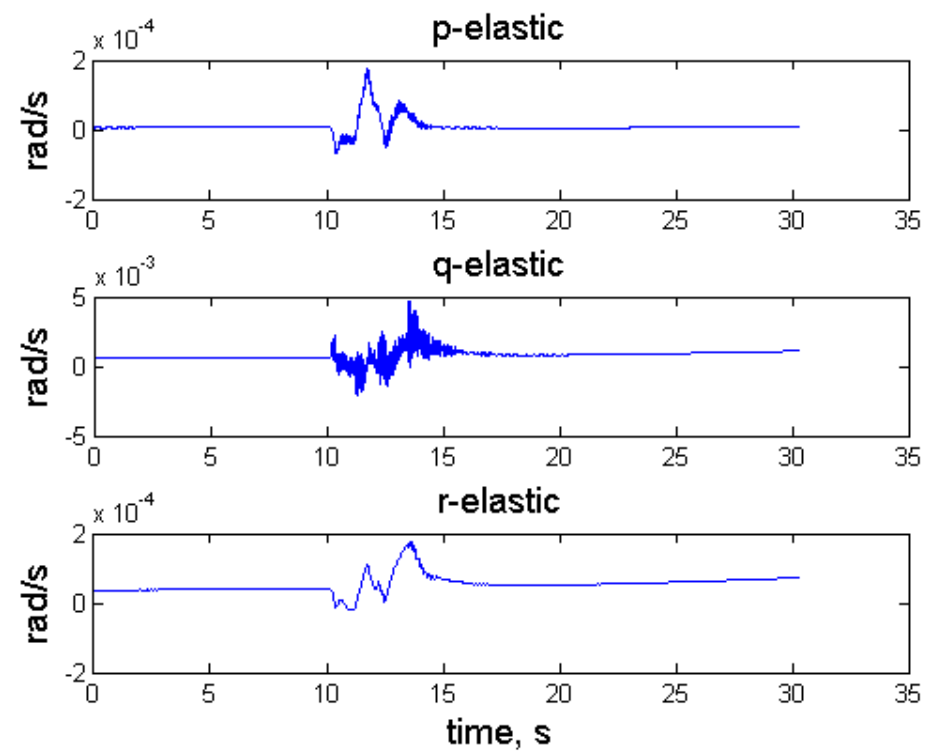

Figure 42: ABCDX Sim Steady State Plots- Elastic Component- Stick Doublet - Roll, Pitch, and Yaw Rates 
Figure 43 shows the percent impact of the elastic roll rate dynamics on the total roll, pitch, and yaw rate dynamics of the aircraft.
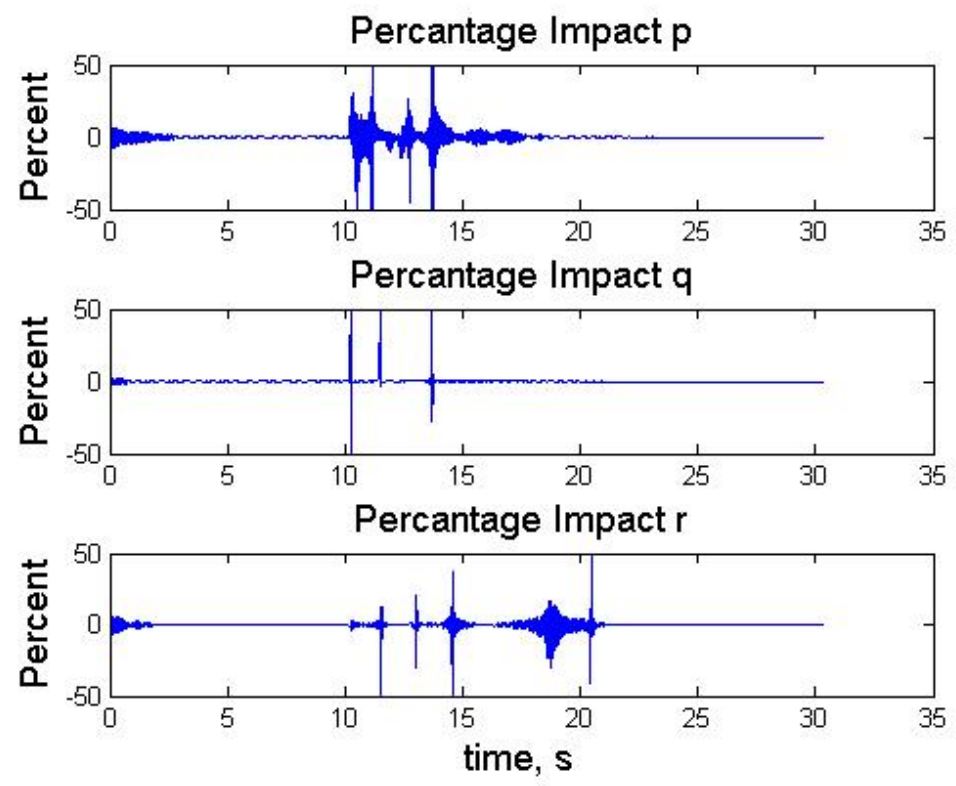

Figure 43: ABCDX Sim Steady State Plots- Elastic Component-Roll, Pitch, and Yaw RatesStick Doublet - Percentage Impact

Figure 44 shows the rigid body contribution to the changing accelerations through time.
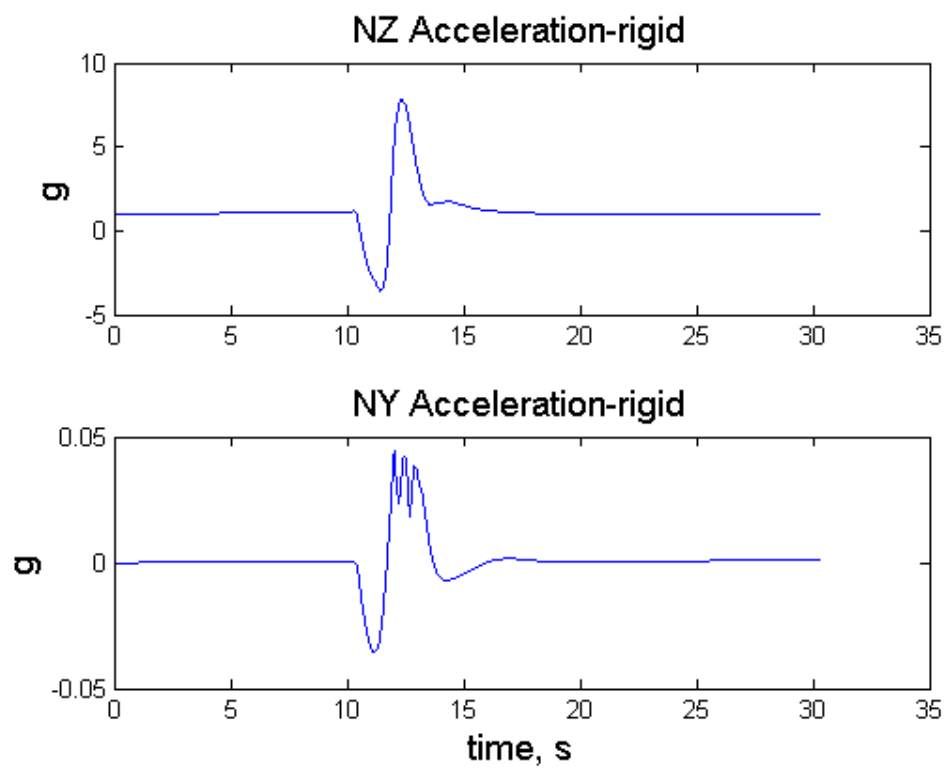

Figure 44: ABCDX Sim Steady State Plots- Rigid - Stick Doublet - Accelerations 
Figure 45 shows the elastic body contribution to the changing accelerations through time.
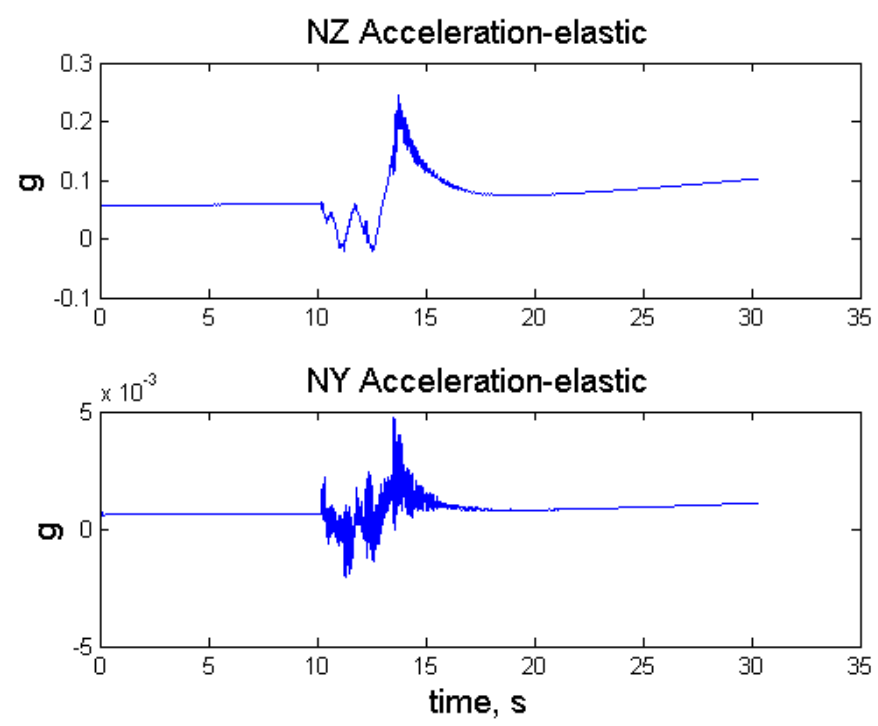

\section{Figure 45: ABCDX Sim Steady State Plots- Elastic Component- Stick Doublet - Accelerations}

Figure 46 shows the percent impact of the elastic acceleration dynamics to the total acceleration dynamics of the aircraft.
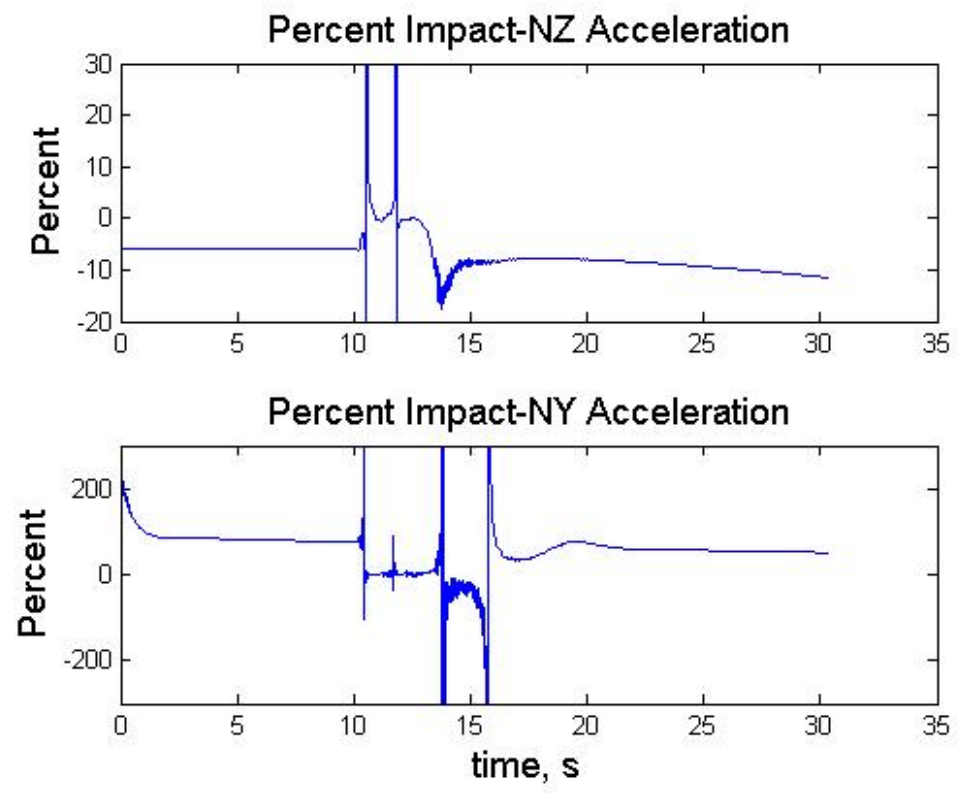

Figure 46: ABCDX Sim Steady State Plots- Elastic Component- Stick Doublet Accelerations- Percentage 
Figure 47 shows the rigid body contribution to the changing roll and pitch angle through time.
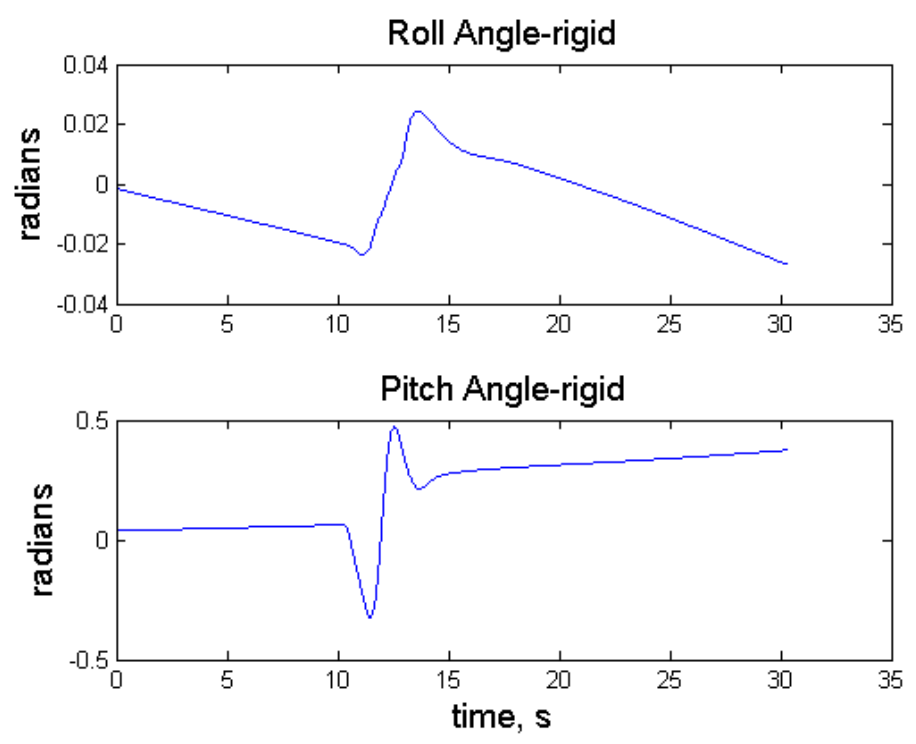

Figure 47: ABCDX Sim Steady State Plots- Rigid - Stick Doublet - Roll and Pitch Angle

Figure 48 shows the elastic body contribution to the changing roll and pitch angle through time.
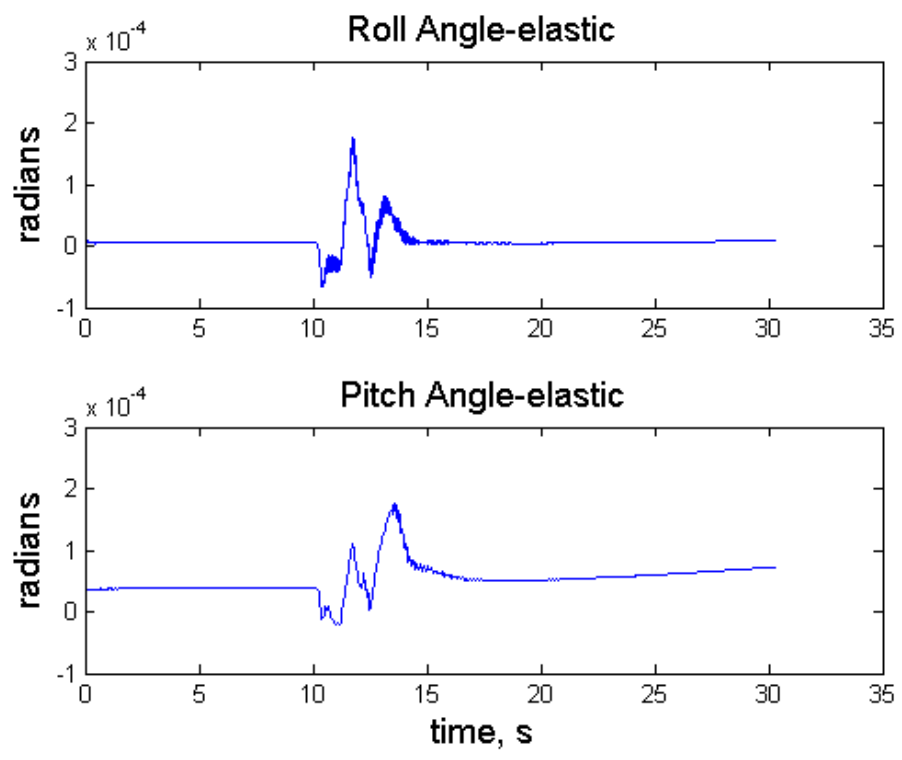

Figure 48: ABCDX Sim Steady State Plots- Elastic Component- Stick Doublet - Roll and Pitch Angles 
Figure 49 shows the percent impact of the elastic roll angle contribution to the total roll angle dynamics of the aircraft.
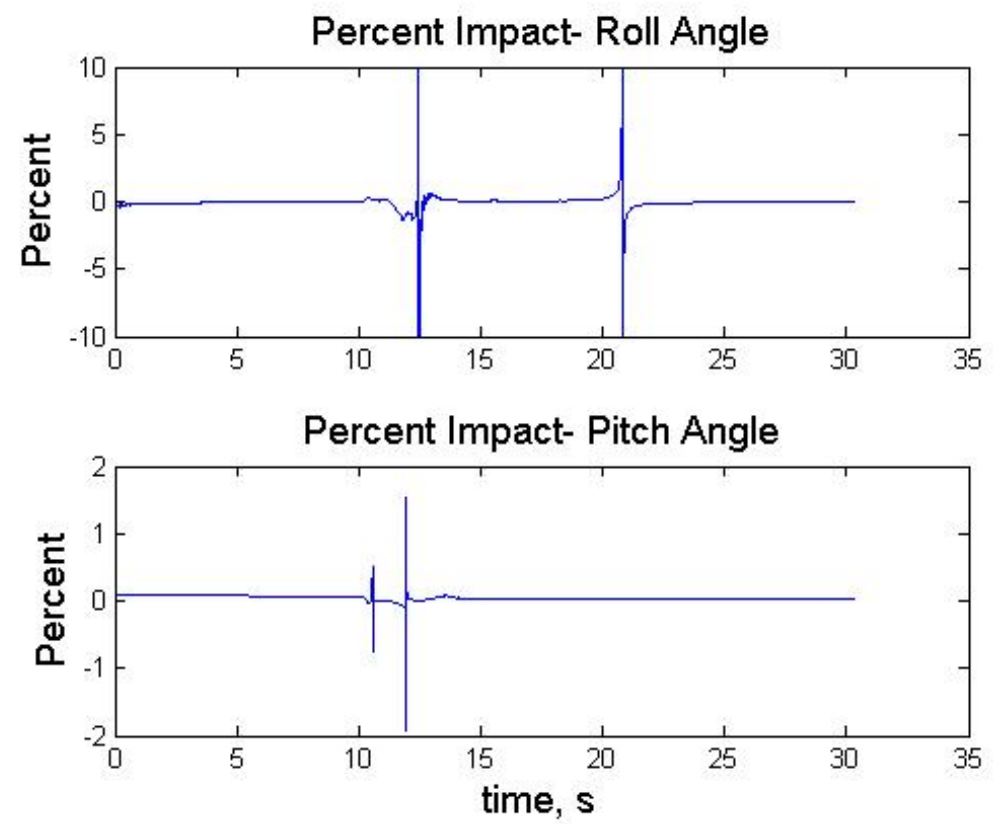

Figure 49: ABCDX Sim Steady State Plots- Elastic Component- Stick Doublet - Roll and Pitch Angles- Percentage

\subsection{Model PLANTX- 30 Seconds- No Pilot Inputs}

Figures 50-59 correspond to using elastic model PLANTX for 30 seconds of flight data allowing the simulation to drift with no pilot inputs. Figure 50 shows the changing altitude and mach flight conditions through time. 

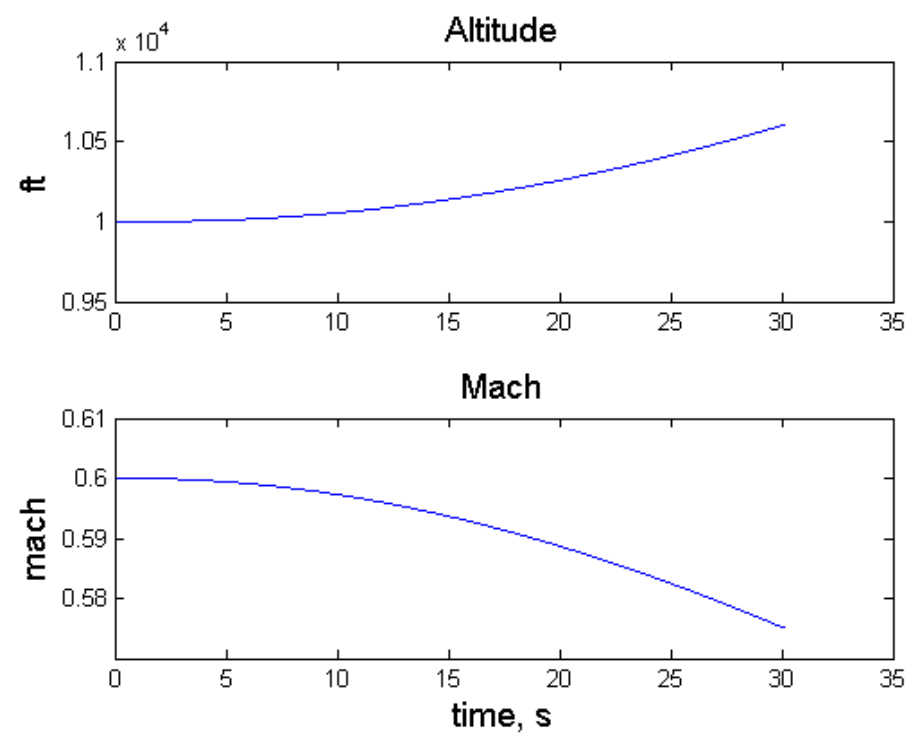

Figure 50: PLANTX Sim Steady State Plots- Flight Conditions

Figure 51 shows the rigid body contribution to the changing roll, pitch, and yaw rates through time.
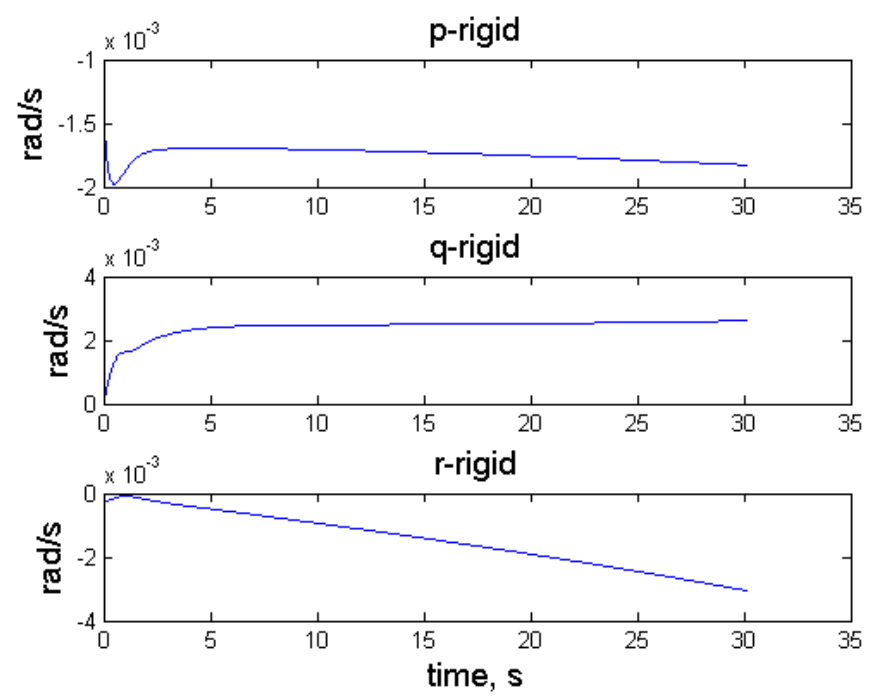

Figure 51: PLANTX Sim Steady State Plots- Rigid- Roll, Pitch, and Yaw Rate 
Figure 52 shows the elastic body contribution to the changing roll, pitch, and yaw rates through time.
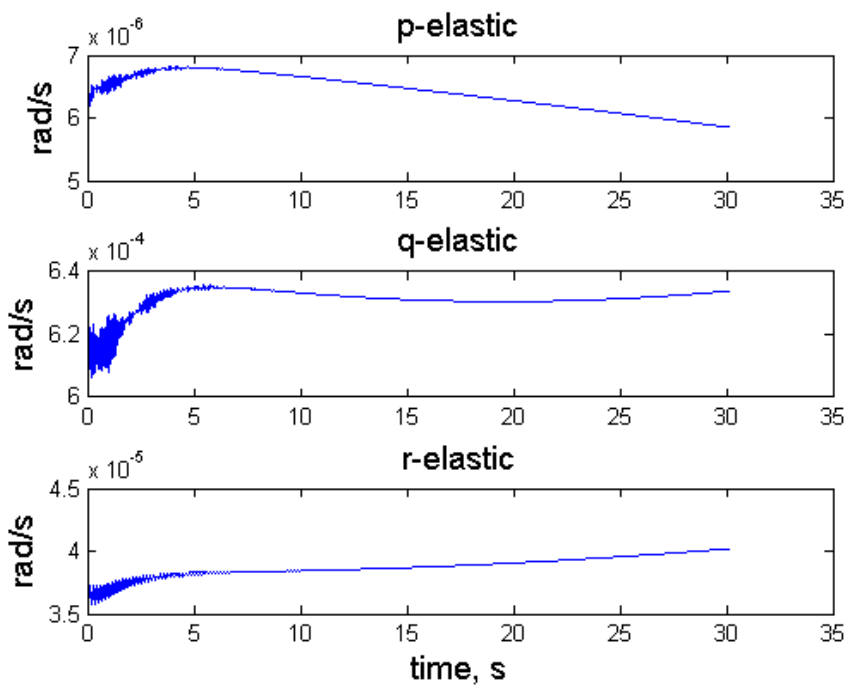

Figure 52: PLANTX Sim Steady State Plots- Elastic Component- Roll, Pitch, and Yaw Rates

Figure 53 shows the percent impact of the elastic roll, pitch, and yaw rate dynamics on the total roll, pitch, and yaw rate dynamics of the aircraft.
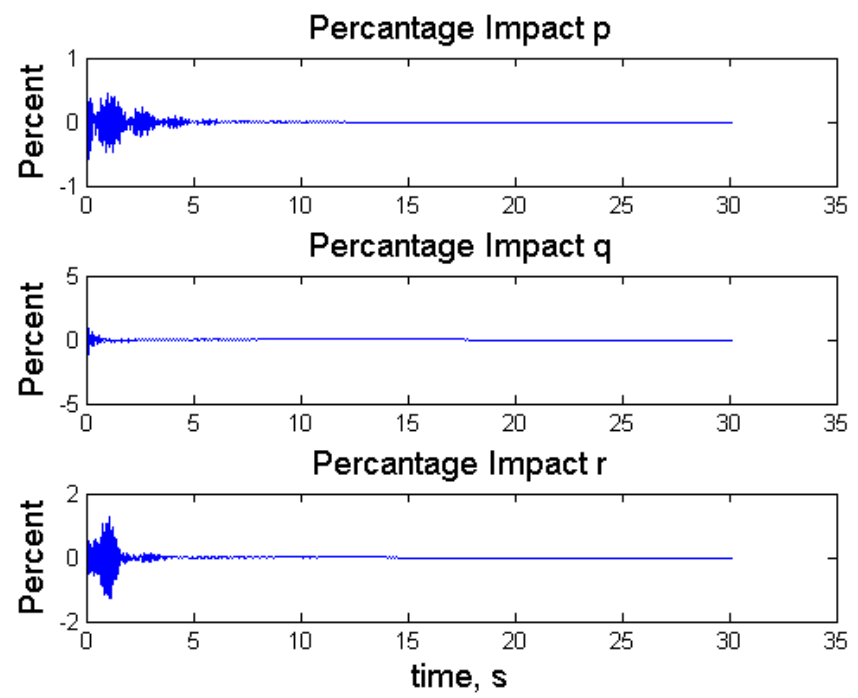

Figure 53: PLANTX Sim Steady State Plots- Elastic Component- Stick Doublet - Roll, Pitch, and Yaw Rates-Percentage Impact

Figure 54 shows the rigid body contribution to the changing accelerations through time. 

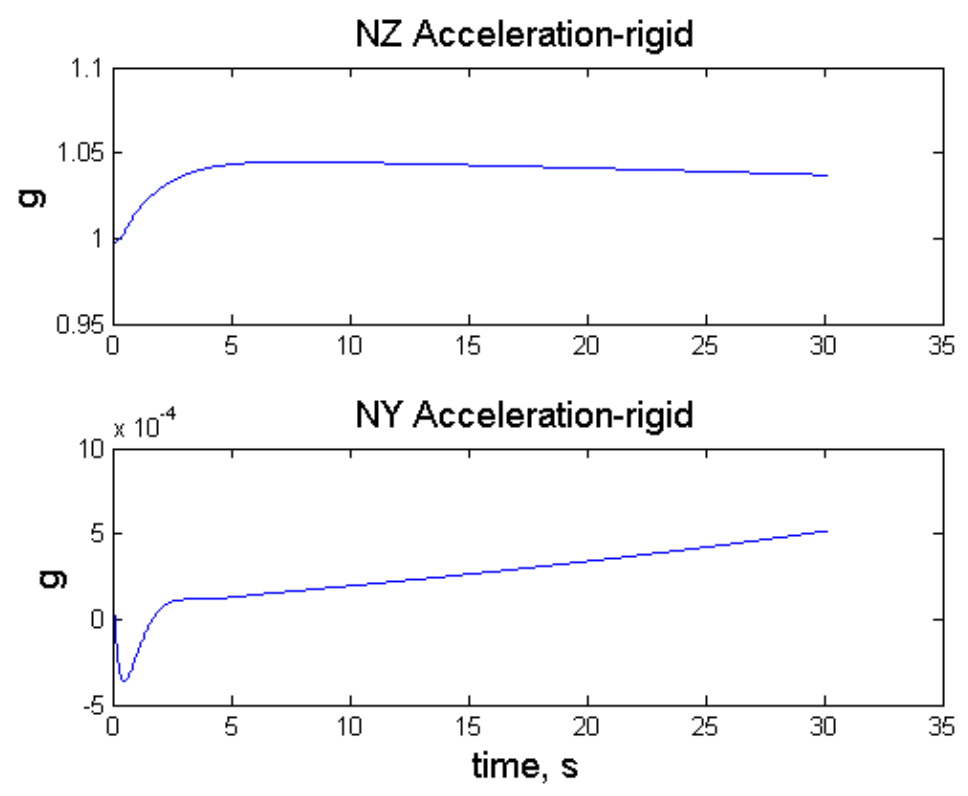

Figure 54: PLANTX Sim Steady State Plots- Rigid -Accelerations

Figure 55 shows the elastic body contribution to the changing accelerations through time.
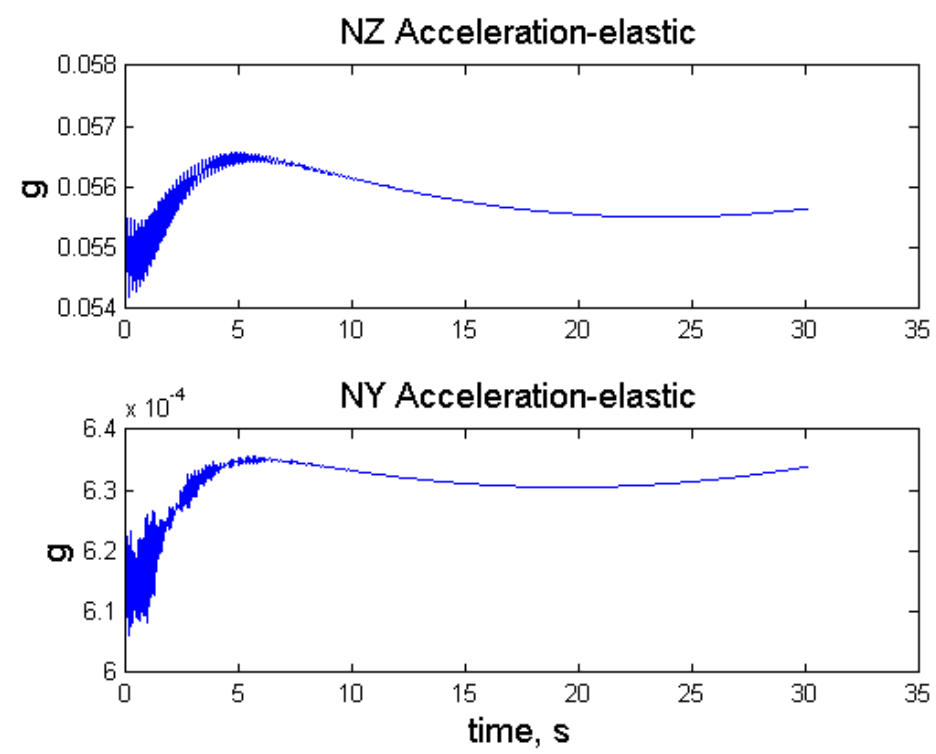

Figure 55: PLANTX Sim Steady State Plots- Elastic Component- Accelerations 
Figure 56 shows the percent impact of the elastic acceleration dynamics to the total acceleration dynamics of the aircraft.
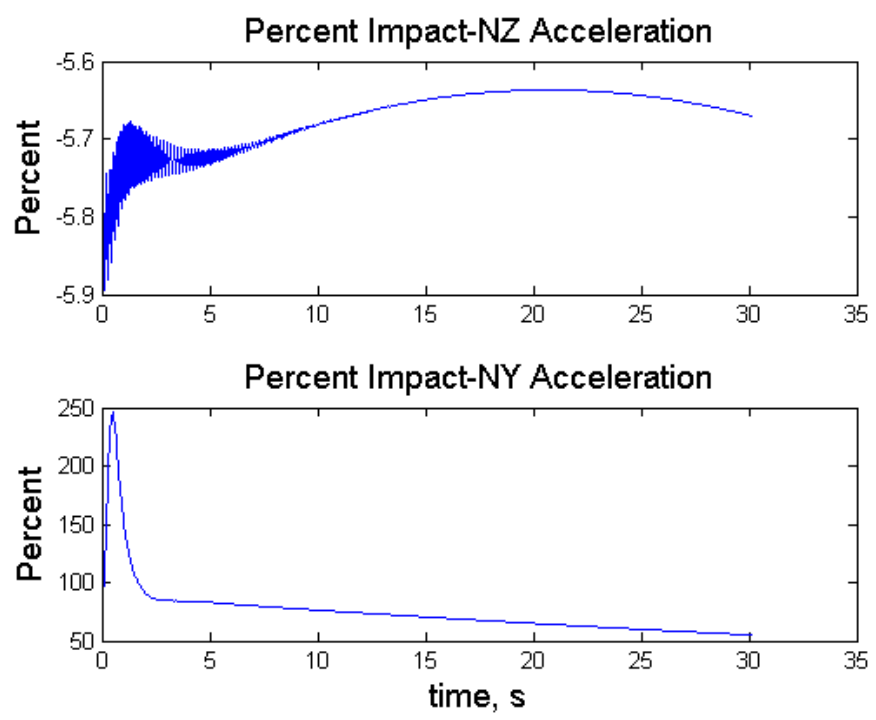

Figure 56: PLANTX Sim Steady State Plots- Elastic Component- Accelerations- Percentage Figure 57 shows the rigid body contribution to the changing roll and pitch angle through time.
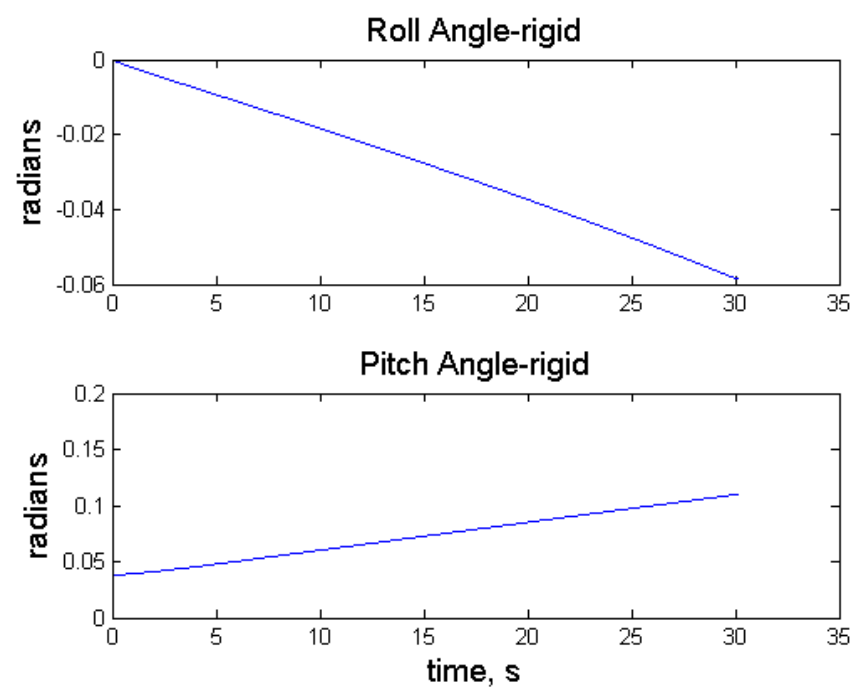

Figure 57: PLANTX Sim Steady State Plots- Rigid - Roll and Pitch Angle 
Figure 58 shows the elastic body contribution to the changing roll and Pitch angle through time.
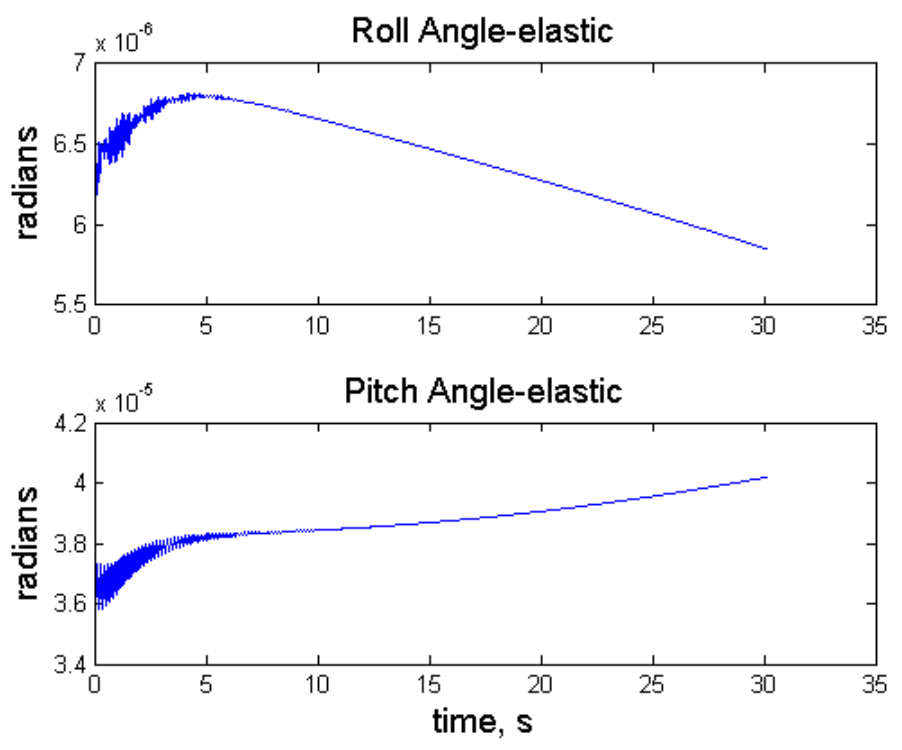

Figure 58: PLANTX Sim Steady State Plots- Elastic Component- Roll and Pitch Angles

Figure 59 shows the percent impact of the elastic roll angle contribution to the total roll and pitch angle dynamics of the aircraft.
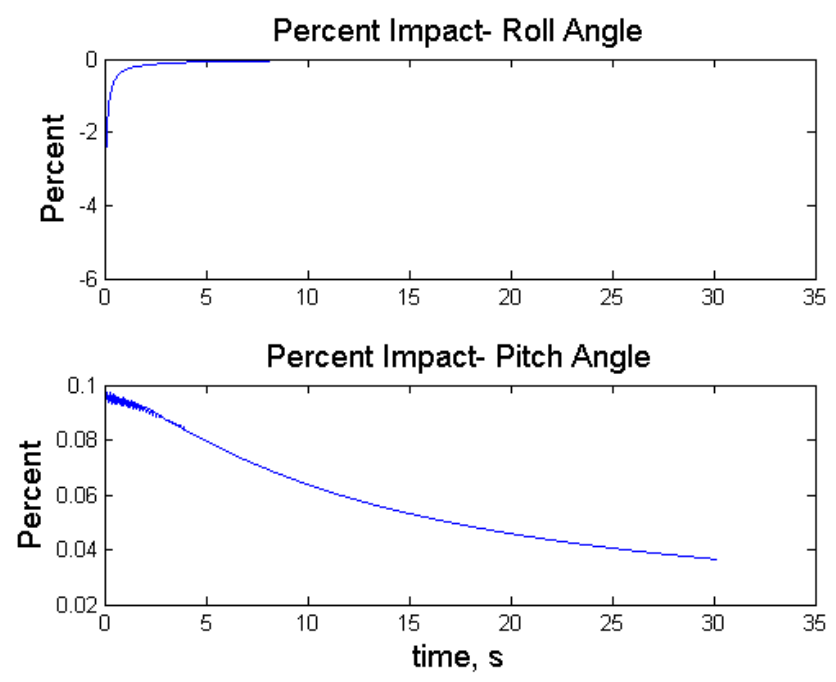

Figure 59: PLANTX Sim Steady State Plots- Elastic Component- Roll and Pitch AnglesPercentage 


\subsection{Model PLANTX- 30 Seconds- Doublet Input}

Figures 60-69 correspond using elastic model PLANTX for 30 seconds of flight data allowing the simulation to drift with a pitch doublet pilot input approximately 12 seconds into the simulation. Figure 40 shows the changing altitude and mach flight conditions through time.
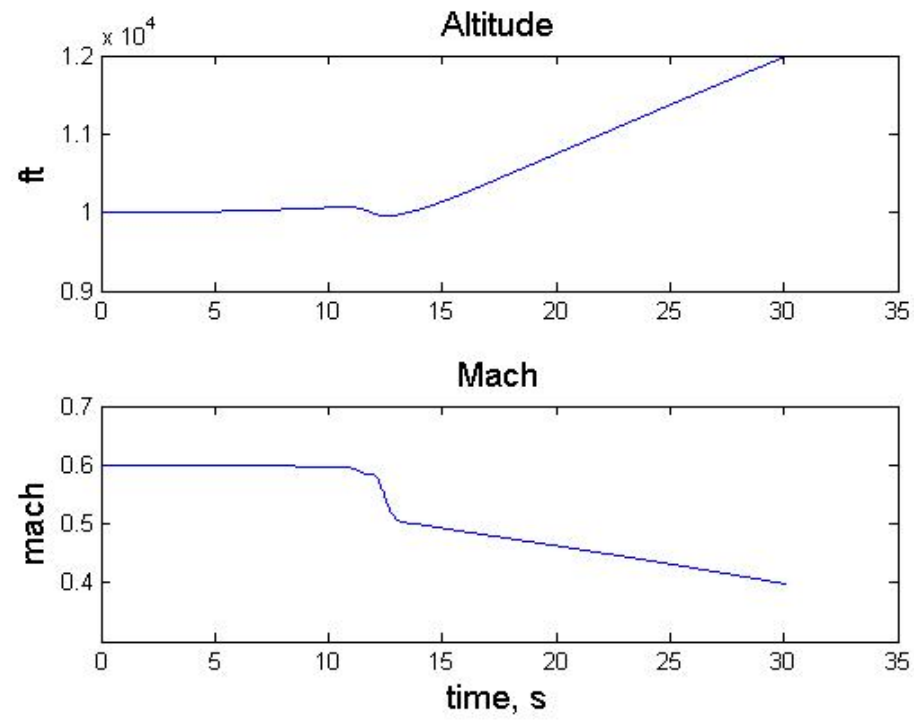

Figure 60: PLANTX Sim Steady State Plots- Flight Conditions

Figure 61 shows the rigid body contribution to the changing roll, pitch, and yaw rates through time. 

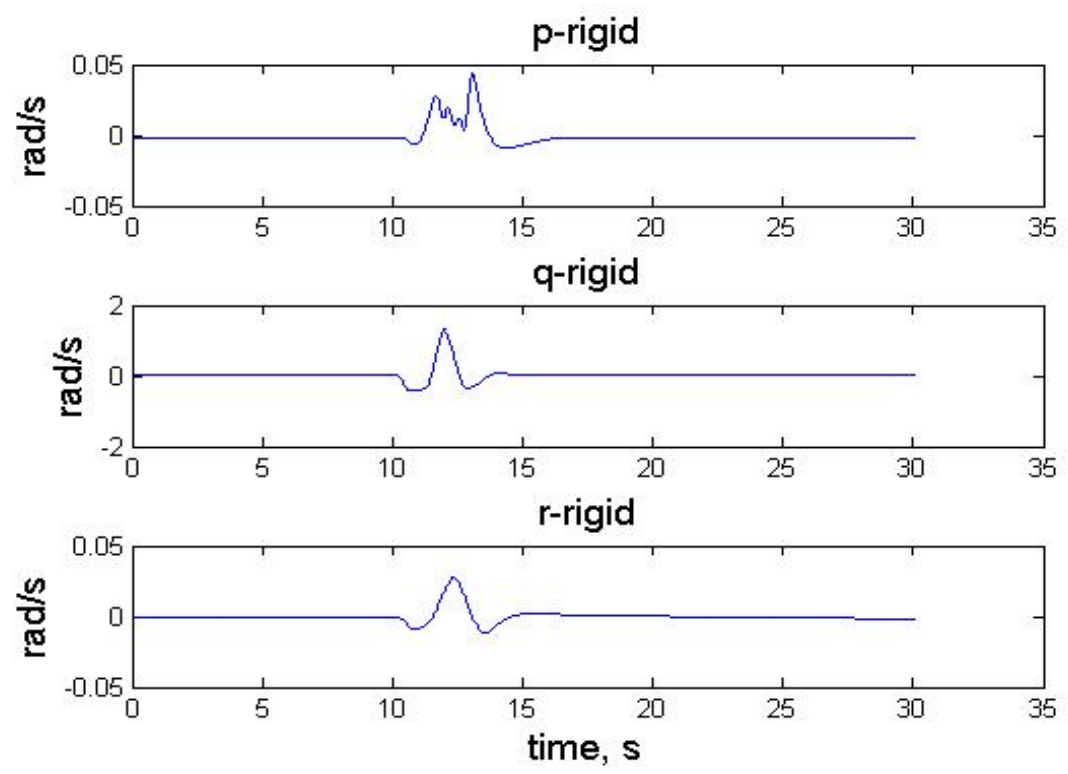

Figure 61: PLANTX Sim Steady State Plots- Rigid- Stick Doublet - Roll, Pitch, and Yaw Rate

Figure 62 shows the elastic body contribution to the changing roll, pitch, and yaw rates through time.
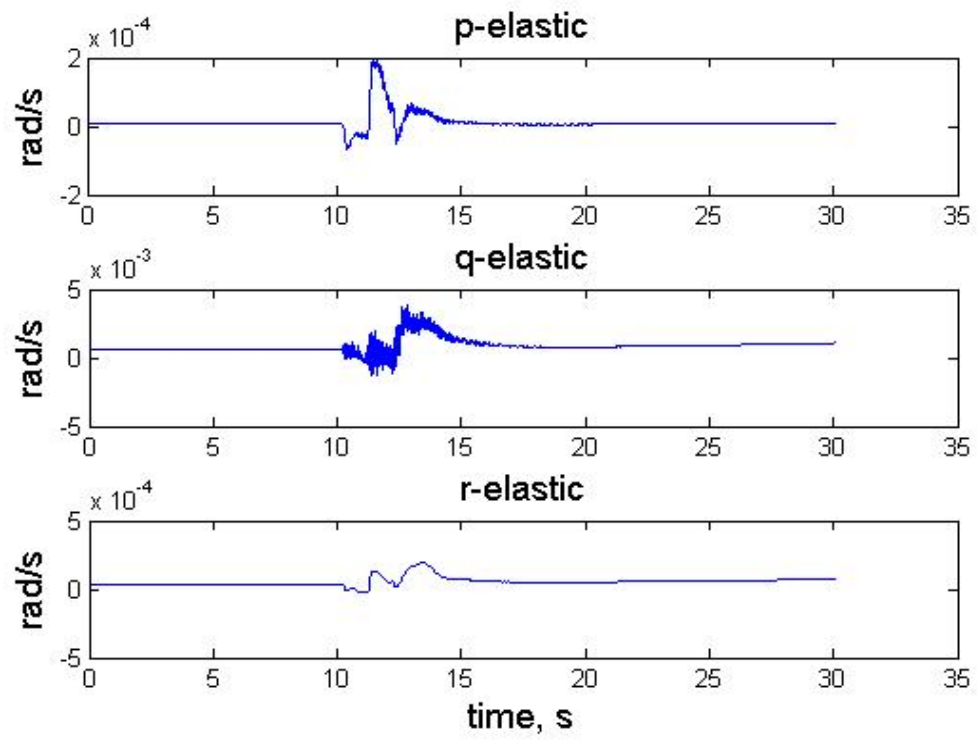

Figure 62: PLANTX Sim Steady State Plots- Elastic Component- Stick Doublet - Roll, Pitch, and Yaw Rates 
Figure 63 shows the percent impact of the elastic roll, pitch, and yaw rate dynamics on the total roll rate the dynamics of the aircraft.
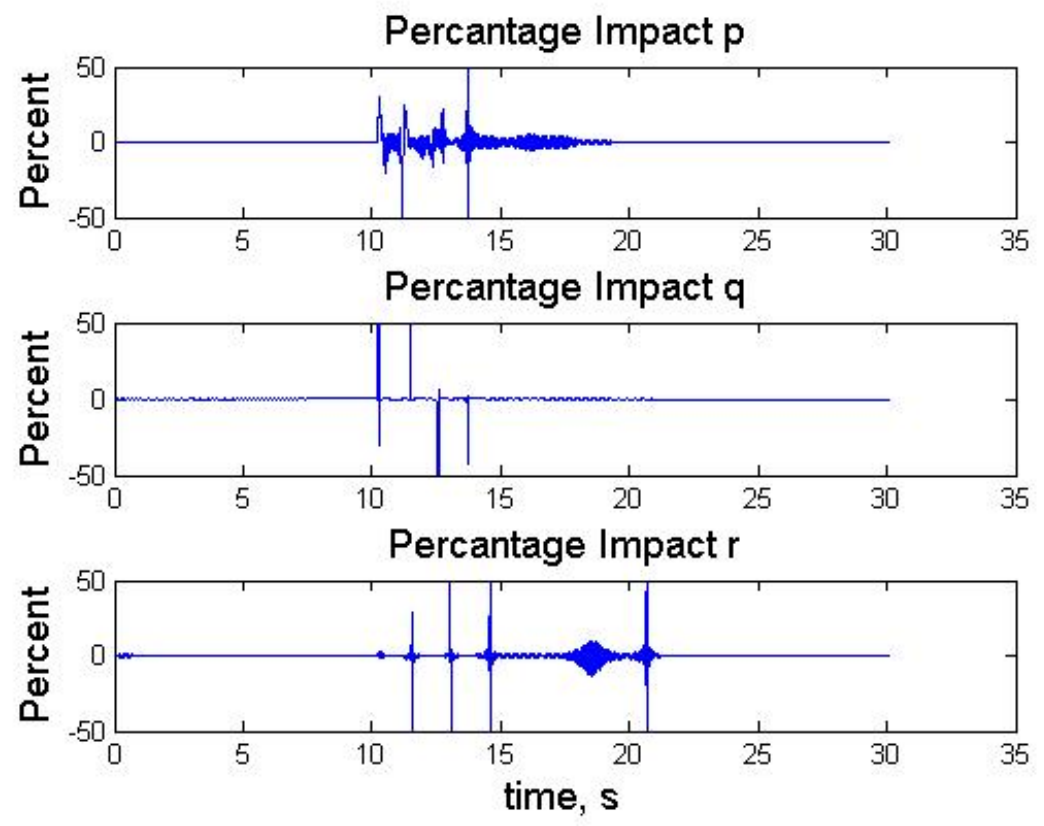

Figure 63: PLANTX Sim Steady State Plots- Elastic Component-Roll, Pitch, and Yaw Rates- Stick Doublet - Percentage Impact

Figure 64 shows the rigid body contribution to the changing accelerations through time.
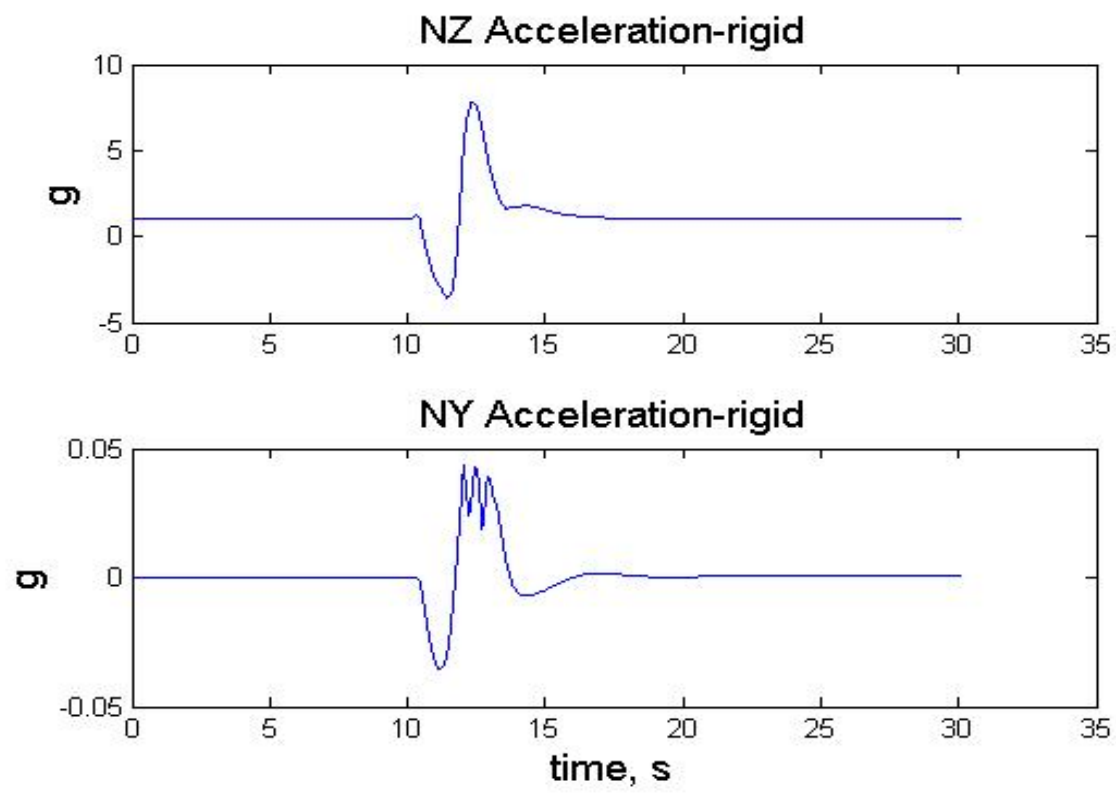

Figure 64: PLANTX Sim Steady State Plots- Rigid - Stick Doublet - Accelerations 
Figure 65 shows the elastic body contribution to the changing accelerations through time.
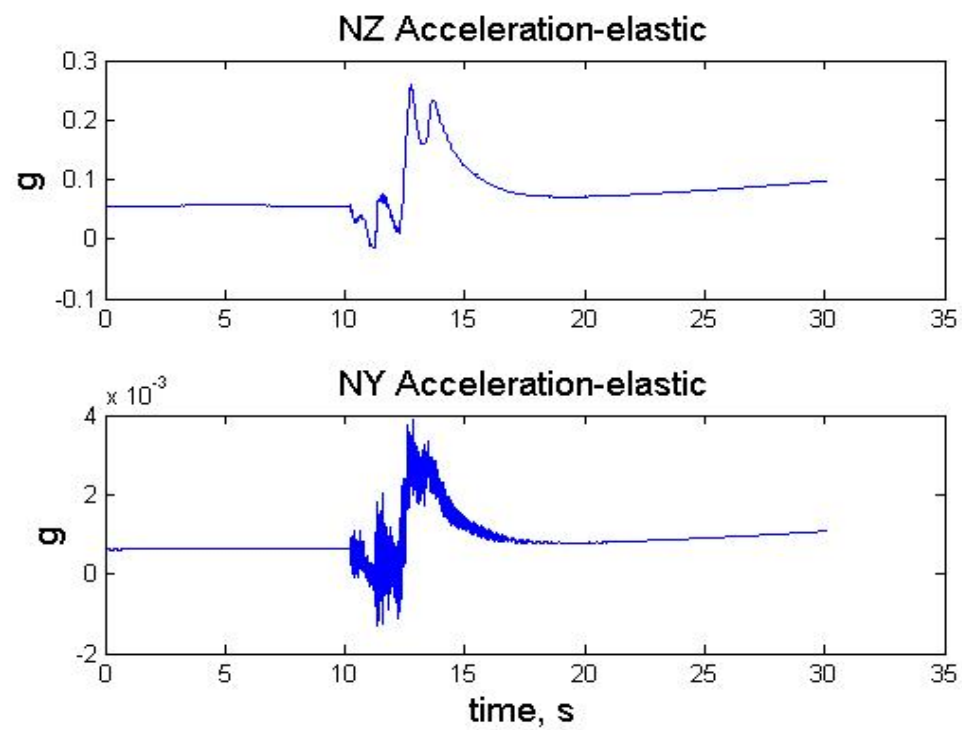

Figure 65: PLANTX Sim Steady State Plots- Elastic Component- Stick Doublet Accelerations

Figure 66 shows the percent impact of the elastic acceleration dynamics to the total acceleration dynamics of the aircraft.
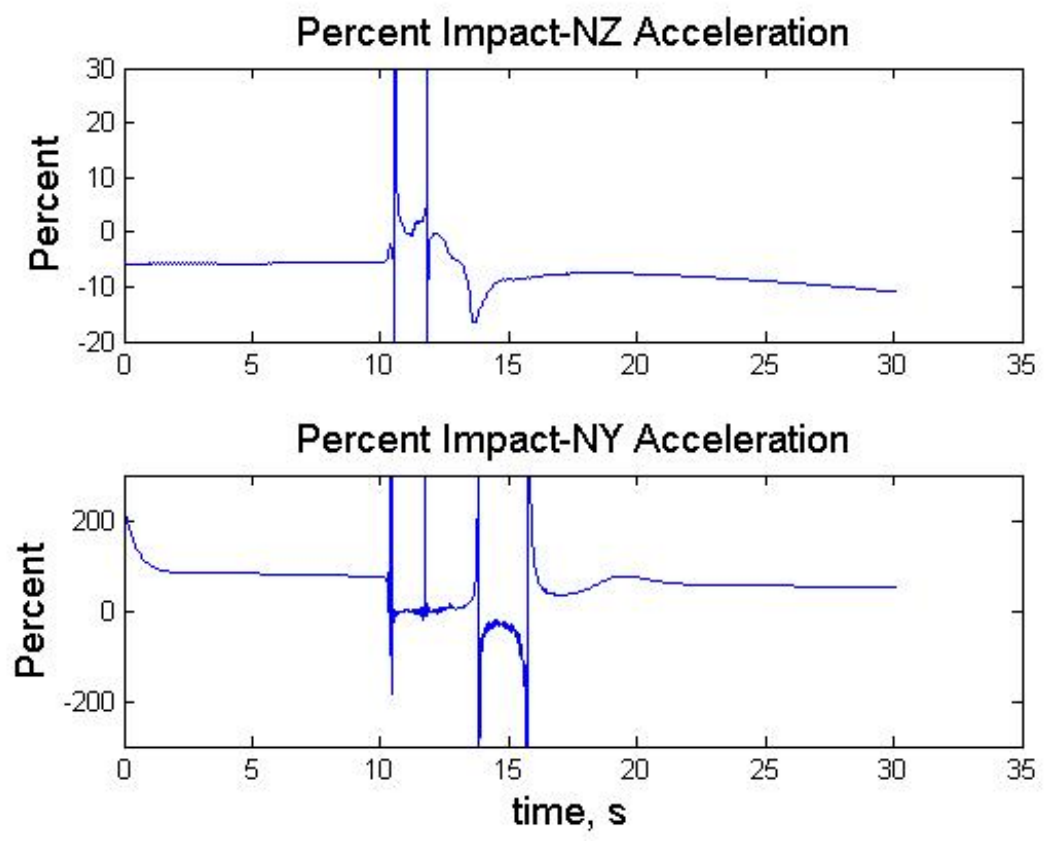

Figure 66: PLANTX Sim Steady State Plots- Elastic Component- Stick Doublet Accelerations- Percentage 
Figure 67 shows the rigid body contribution to the changing roll and pitch angle through time.
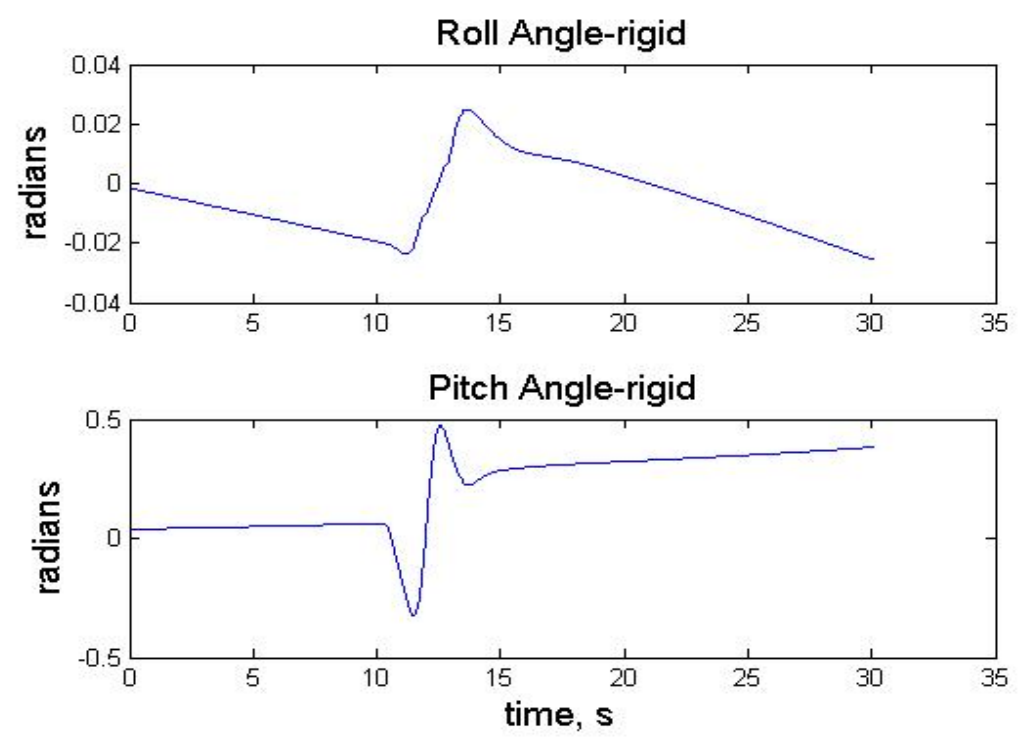

Figure 67: PLANTX Sim Steady State Plots- Rigid - Stick Doublet - Roll and Pitch Angle

Figure 68 shows the elastic body contribution to the changing roll and pitch angle through time.
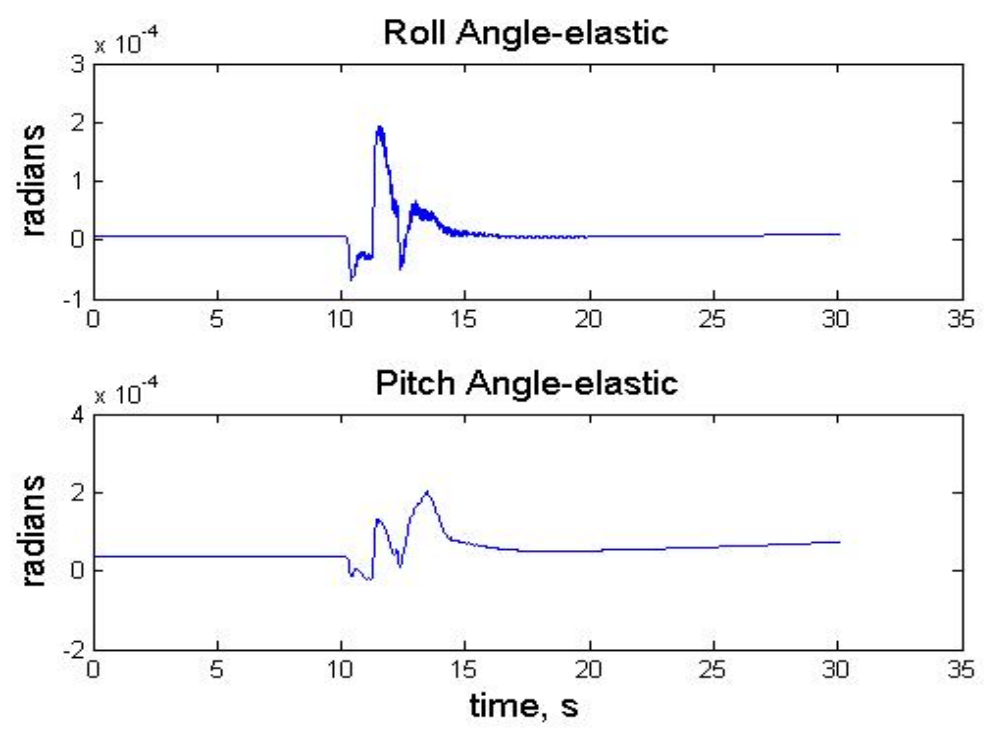

Figure 68: PLANTX Sim Steady State Plots- Elastic Component- Stick Doublet - Roll and Pitch Angles

Figure 69 shows the percent impact of the elastic roll angle contribution to the total roll and pitch angle dynamics of the aircraft. 

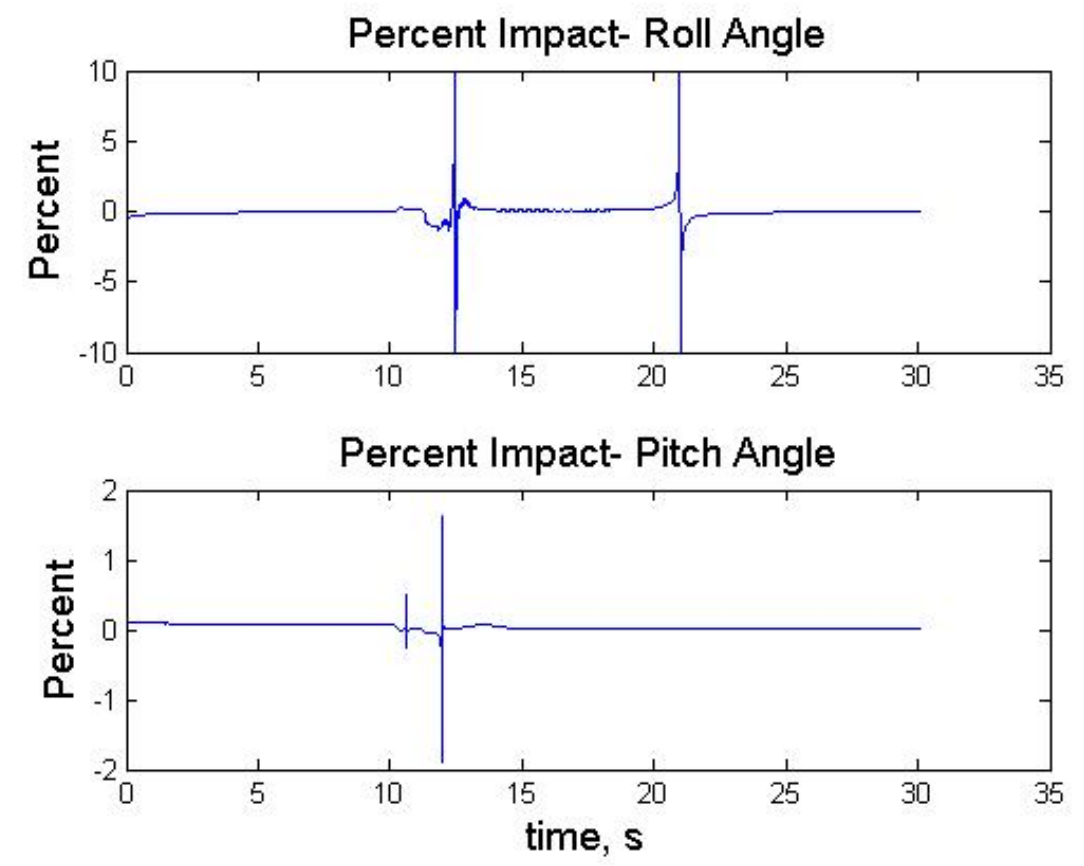

Figure 69: PLANTX Sim Steady State Plots- Elastic Component- Stick Doublet - Roll Angles- Percentage 


\section{Chapter 7}

\section{Conclusions}

\subsection{Summary of Process}

The aeroservoelastic models derived from the Active Aeroelastic Wing Program models were provided for 25 flight conditions. This information was expanded through interpolation and duplication to fill a reference grid to encompass the entire expected flight envelope of the F/A-18 in the simulator. In order to integrate the ASE models into the digital simulator, all models were discretized in Matlab and organized into a three dimensional matrix for transfer into the FORTRAN environment. The ASE models were connected to the corresponding inputs and outputs of the simulator. Based upon the flight conditions of the simulator the appropriate ASE models were called and interpolated to fit the exact flight condition. This interpolated ASE model is then used to calculate the ASE dynamics at the specific flight condition.

Results came out as expected. Aside from accelerations, elastic impacts on the total flight dynamics accounted for less than five percent of the total effects. Flight dynamics for using the 
ABCDX model and PLANTX model were similar and consistent with each other increasing the confidence in their accuracy and usage of each.

The framework for ASE model integration in the NASA Dryden F/A-18 simulator has been established. As improved ASE models are developed, using the established process from this thesis should simplify the integration process. In addition, referencing this methodology can help support the development of future unified rigid body and elastic models for other aircraft, thus increasing the simulation's accuracy in imitating real world dynamics.

\subsection{Overview of Results}

The sim remained stable and controllable after integration of ASE models. In most cases, the percentage contribution impact from ASE outputs compared to rigid body outputs is small at less than five percent, except for the Ny and Nz components. Since rigid body acceleration contributions to the flight dynamics are very small already, it is reasonable that the elastic accelerations would have a much larger contribution to the total flight dynamics. In conditions where pitch doublets were inserted into the simulation, brief spikes from the elastic models affected the sim, but were quickly dampened out.

Outputs between the two elastic models; ABCDX and PLANTX were very similar to each other. This helped verify the accuracy of the coupled actuator models with the actuator models in the PLANTX model. This was true for both cases when a doublet was and was not applied. 


\subsection{Future Recommendations}

Obtaining additional flight data throughout the flight envelope will ensure more accurate ASE characterizations. This will improve the accuracy of the interpolations since real flight data would be increasingly used.

Next steps should focus on comparing simulation flight dynamics with real world dynamics to verify the accuracy of the combined rigid body and elastic model. In addition, input provided from pilots would be valuable to record if differences are noticed in the flight responses between the simulation and the actual aircraft during flight.

Ultimately, implementing unified rigid body and elastic models into simulations for other aircraft beyond the F/A-18 will provide valuable flight dynamics behavior that better characterizes real world environments. The F/A-18 simulation framework will provide a basis for these future endeavors and assist in future research in the fields of structural dynamics and aeroservoelasticity to accurately test aircraft modifications in simulation. 


\section{Bibliography:}

[1] Wright, J.R. Cooper, J.E. "Introduction to Aircraft Aeroelasticity and Loads.” John Wiley \& Sons, Ltd. England. 2007.

[2] Curlett, B.P. “A Software Framework for Aircraft Simulation.” NASA TM-214639, 2008.

[3] Megson, T.H.G. "Aircraft Structures for Engineering Students.” Elsevier, Singapore. 1998.

[4] Dowell, E.H. Curtiss, H.C. Jr., Scanlan, R.H. Sisto, F. “A Modern Course in Aeroelasticity.” John Wiley \& Sons. 1978.

[5] Bisplinghoff, R. L., Ashley, H., and Halfman, R. L. “Aeroelasticity.” AddisonWesley, Cambridge,MA, 1955.

[6] Fung Y.C. “An Introduction to The Theory Of Aeroelasticity.” John Wiley \& Sons. New York. 1955.

[7] Bispilnghoff, R.L. Ashley, H. "Principles of Aeroelasticity," Dover Publications, Inc. New York. 1962

[8] Teng, Y. “An Introduction to Flutter, Aeroservoelasticity \& Active flutter Control.” Accessed September $7^{\text {th }}$ 2010. http://yingteng.tripod.com/AFS.pdf 
[9] Lind, R., Brenner, M. “Robust Aeroservoelastic Stability Analysis.” Springer-Verlag, London, April 1999.

[10] Moore, R.L. "Aeroservoelastic Stability Analysis of an Airplane with a Control Augmentation System.” Ph.D. Dissertation, The Ohio State University, 1978, Available from University Microfilms International, No.7902191.

[11] Brenner M.J. “Aeroservoelastic Uncertainty Model Identification from Flight Data.” NASA TM-210397, 2001.

[12] Allen, M.J., Lizotte, A.M., Dibley, R.P., Clarke, R. “Loads Model Development and Analysis for the F/A-18 Active Aeroelastic Wing Airplane.” AIAA-2005-6313. AIAA Atmospheric Flight Mechanics Conference and Exhibit, San Francisco, CA, August 15$18,2005$.

[13] Olney, C.D., Hillebrandt, H., Reichenbach, E.Y. “An Evaluation Technique for an F/A18 Aircraft Loads Model Using F/A-18 Systems Research Aircraft Flight Data." NASA/TM-2000-209028, 2000.

[14] Diebler, C.G., Cumming, S.B. “Active Aeroelastic Wing Aerodynamic Model Development and Validation for a Modified F/A-18A Airplane.AIAA-2005-6312. AIAA Atmospheric Flight Mechanics Conference and Exhibit. San Francisco, CA, August 15$18,2005$.

[15] Pendleton, E., Bessette, D., Field, P., Miller, G., Griffin, K. “The Active Aeroelastic Wing Flight Research Program Technical Program \& Model Analytical Development.” AIAA-98-1972, 1998.

[16] Dibley, R.P., Allen, M.J., Clarke, R. "Development and Testing of Control Laws for the Active Aeroelastic Wing Program.” NASA/TM-2005-213666, 2005. 
[17] AAW Testbed Operating Envelope Figure. The Boeing Company. Accessed September $7^{\text {th }}, 2010$. http://www.boeing.com/assocproducts/aaw/figure1.htm

[18] Norlin, K.A. "Flight Simulation Software at NASA Dryden Flight Research Center." NASA TM-104315, 1995.

[19] de Callafon, R.A., Miller, D.N., Brenner, M.J. “Step-Based Experiment Design and System Identification for Aeroelastic Dynamic Modeling." AIAA 2009-5707. AIAA Atmospheric Flight Mechanics Conference. Chicago, IL, August 10-13, 2009.

[20] Lind, R., Brenner, M., "Incorporating Flight Data into a Robust Aeroelastic Model," Journal of Aircraft, Vol. 35, No. 3, 1998, pp. 470-477.

[21] Brenner, M.J. Prazenica, R.J. “Aeroservoelastic Model Validation and Test Data Analysis of the F/A-18 Active Aeroelastic Wing." NASA TM-212021, 2003.

[22] Gupta, K. K., Brenner, M. J., and Voelker, L. S. “Development of an Integrated Aeroservoelastic Analysis Program and Correlation with Test Data." NASA TP3120,May 1991.

[23] Franklin, G.F., Powell, D.J. “Digital Control of Dynamic Systems.” Addison-Wesley Publishing Co., Philippines, 1980.

[24] Brenner, M.J., “Aeroservoelastic Modeling and Validation of a Thrust-Vectoring F/A18 Aircraft.” NASA Technical Paper 3647, September 1996.

[25] Aeroelasticity Image. University of Liverpool. Accessed August 30 ${ }^{\text {th }}, 2010$. http://www.liv.ac.uk/flightscience/PROJECTS/CFD/AEROELASTICITY/index.htm

[26] Livne, E. "Future of Airplane Aeroelasticity." Journal of Aircraft. Vol. 40, N.6, November-December 2003. 
[27] Voracek, D., Pendleton, E., Griffin, K., Reichenbach, E., Welch, L. “The Active Aeroelastic Wing Phase I Flight Research Through January 2003.” NASA/TM-2003$210741,2003$.

[28] Noll, T.E., Perry III, B., Tiffany, S.H., Cole, S.R., Buttrill C.S., Adams Jr., W.M., Houck, J.A., Srinathkumar, S., Mukhopadhyay, V., Pototzky, A.S., Heeg, J., McGraw, S.M.,Miller, G., Ryan, R., Brosnan, M., Haverty, J., and Klepl, M. “Aeroservoelastic Wind-Tunnel Investigations Using the Active Flexible Wing Model - Status and Recent Accomplishments.” NASA TM-101570, 1989.

[29] Livne, E., "Integrated Aeroservoelastic Optimization: Status and Progress", Journal of Aircraft, Vol. 36, No. 1, January-February 1999, pp. 122-145.

[30] Collar, A. R. “The First Fifty Years of Aeroelasticity.” Aerospace (Royal Aeronautical Society Journal), February 1978.

[31] Waszak, R.R. Buttrill, C.S. Schmidt, D.K. Modeling and Model Simplification of Aeroelastic Vehicles: An Overview. NASA Technical Memorandum 107691. September, 1992.

[32] Matlab user guides: MathWorks, Aerospace Blockset, User’s Guide, The MathWorks, Inc., Natick, MA 01760-2098, version 2 ed., March 2006. 


\section{Appendix A: Double Linear Triangular Interpolation Derivation}

\section{A.1 Insufficient Interpolation Methods}

\section{A.1.1 Triangular Interpolation:}

Triangular interpolation assumes that all four data points are all co-planar. When all four data points are co-planar, only three points are needed for a correct interpolation. However, the four data points for ASE interpolation are not all co-planar making this interpolation scheme insufficient as shown in Figure 70.
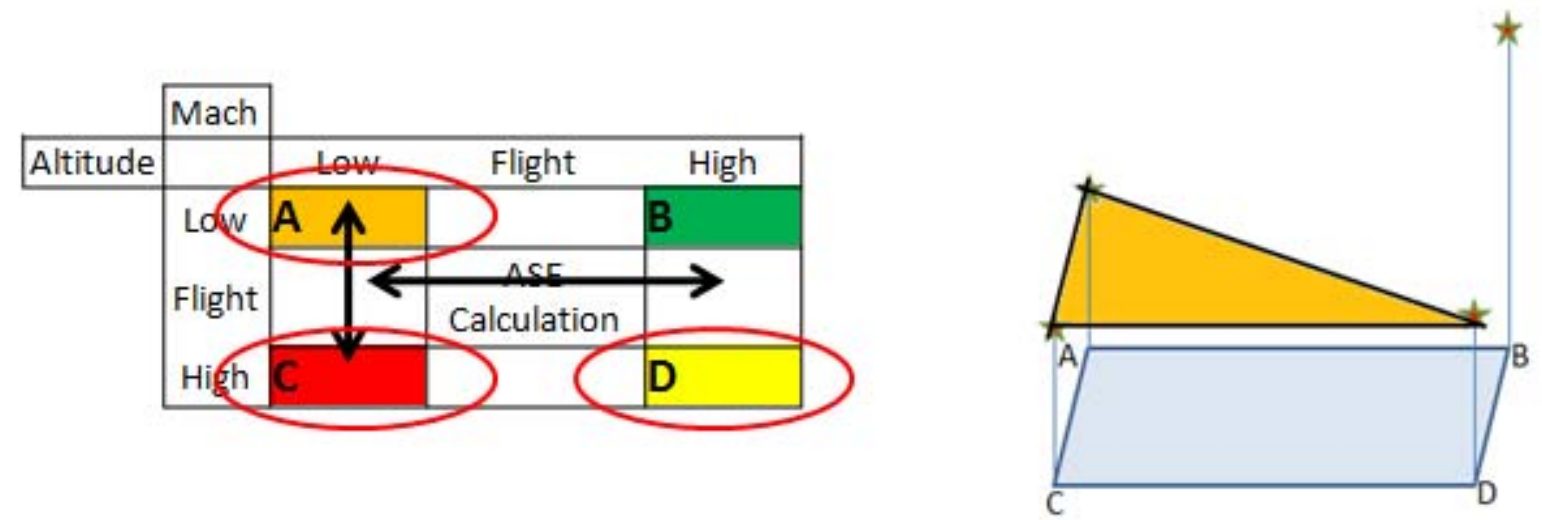

Figure 70: Triangular Interpolation Assumes All Four Data Points are Co-Planar

\section{A.1.2 Bi-linear interpolation:}

Bi-linear interpolation performs a total of three linear interpolations. First interpolations are performed with respect to a constant mach or constant altitude creating two new sets of data. These new data sets are then interpolated to the desired mach or altitude. Although Bi-linear interpolation seems to linearly account for data from all four points, the process actually 
becomes a quadratic interpolation and is no longer a completely linear process. This concept is shown in Figure 71.

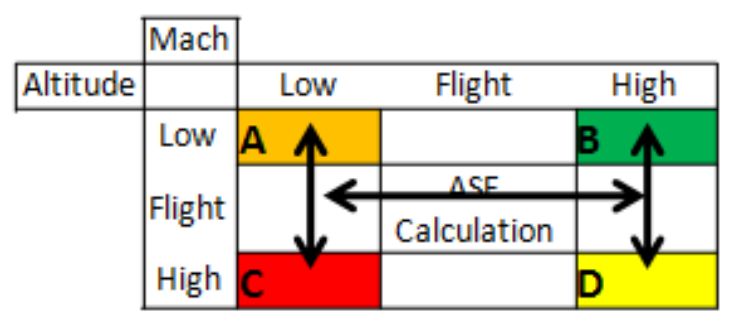

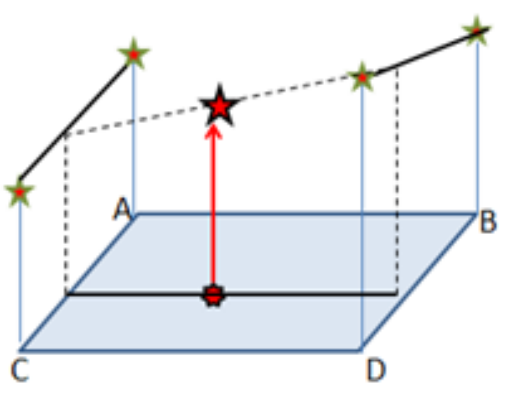

Figure 71: Bi-linear Interpolation is a Quadratic Interpolation Scheme

\section{A.2 Derivation of Double Linear Triangular Interpolation}

\section{A.2.1 Description of Concept:}

An interpolation method called double linear triangular interpolation (DLTI) was developed to linearly interpolate four points in space organized on a regular two dimensional grid. All four data points are used to perform an accurate weighted interpolation calculation.

For any three points, a plane is created where all points must be in the same plane. However, a fourth point does not necessarily have to lie in the same plane as the other three points, which was the case with triangular interpolation. Therefore, an interpolation volume is formed by the tetrahedron created by the four reference points of interest. This tetrahedron is shown in Figure 72. 


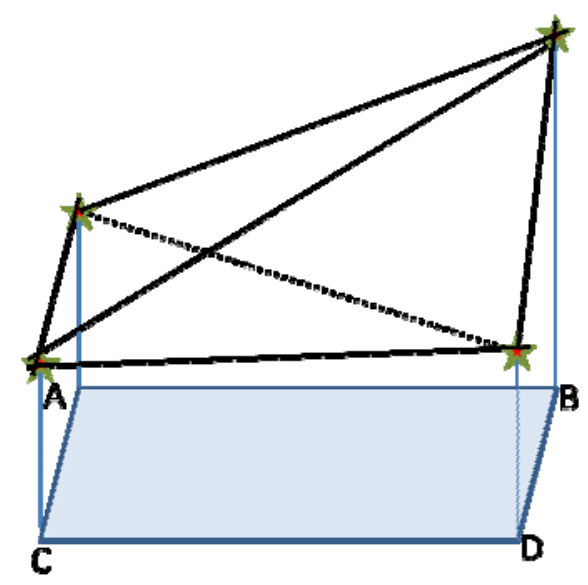

Figure 72: Triangular Prism

These four points of the triangular prism also create four linear planes that correspond to any three points of the triangular prism. These planes act as the boundary edges of the triangular prism as shown in Figure 73.

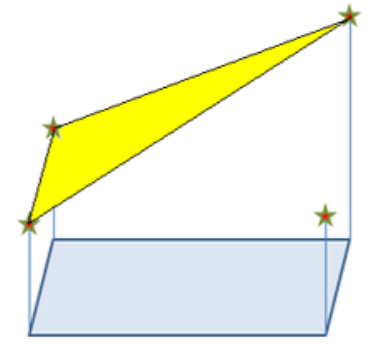

Plane 1

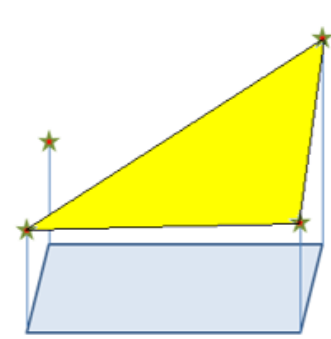

Plane 2

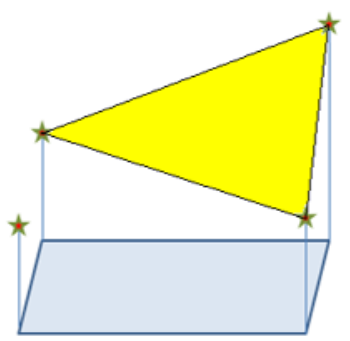

Plane 3

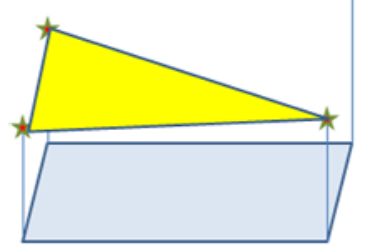

Plane 4

Figure 73: Diagram of Four Planes

In addition, these four points are not completely unbounded to each other. They are tied to known equally spaced coordinates projected on a 2D plane of equally incremented mach and altitude values, such that only their heights are different. This $2 \mathrm{D}$ projection is shown in Figure 74. 


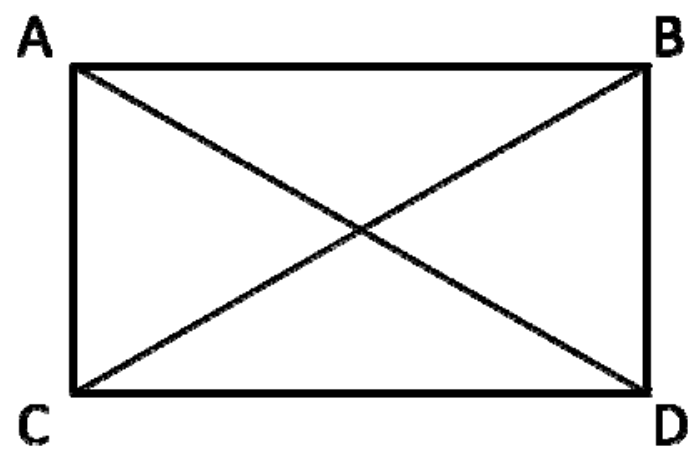

Figure 74: 2D Plane Intersection

\section{A.2.2 Process Overview:}

The four points have known locations on a 2D plane creating a rectangle. This information can be used to determine which planes an interpolation point will pass through. The edges of the tetrahedron must all project onto the 2D plane in such a way that divides the 2D planar rectangle into 4 parts connected diagonally from corner to corner.

To find the value (height) of an interpolated value between the four points, one can create a line from the 2D plane up through the tetrahedron. The line will pass through two and only two planes of the tetrahedron (unless it crosses an edge or corner) that are part of the tetrahedron. Technically, the line will also pass through the other two planes, but only as projections outside of the tetrahedron. This concept is shown in Figure 75.

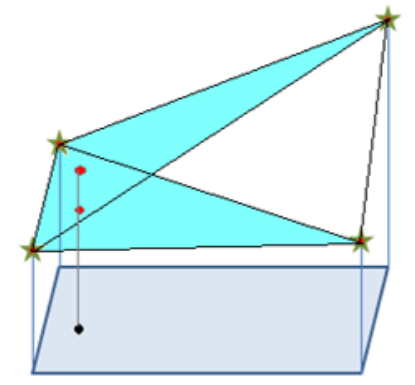

Plane 14

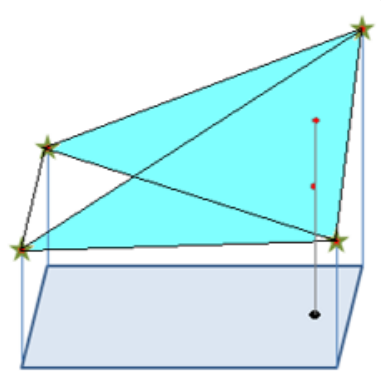

Plane 23

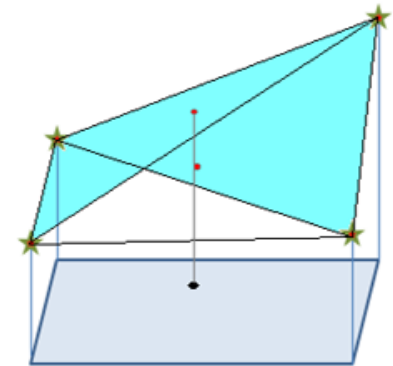

Plane 13

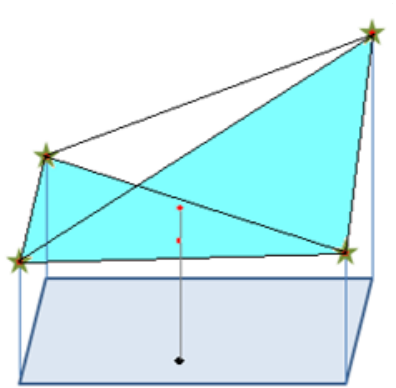

Plane 24

Figure 75: Base Line Cutting Through Two Planes of the Triangular Prism 
Based on the locations for the interpolation data point, we can determine which two planes the point will pass through that is part of the triangular prism of stability. Since we know that these two planes will always have one point on top of the other, we only need to take the average value of the two to find the center point. First, we must perform normal three point triangular interpolations, defined by the two planes that the interpolated point is going through. Taking the average value of these two points will yield the 3D interpolated value. The generalized averaging process for the entire triangular prism is shown in Figure 76.

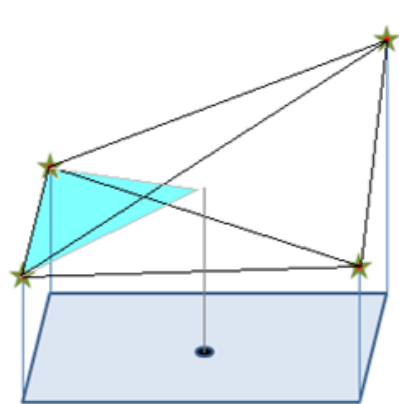

Plane 14

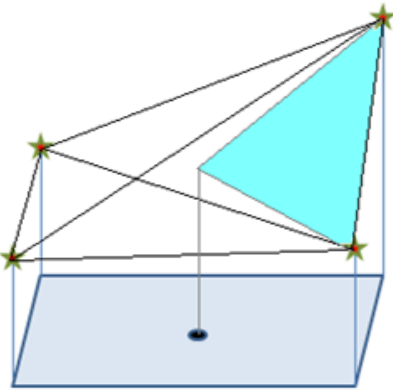

Plane 23

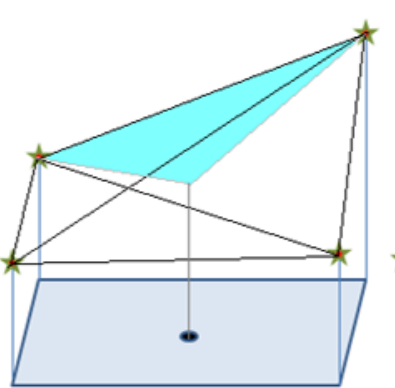

Plane 13

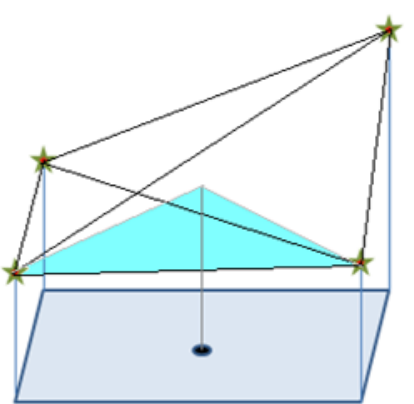

Plane 24

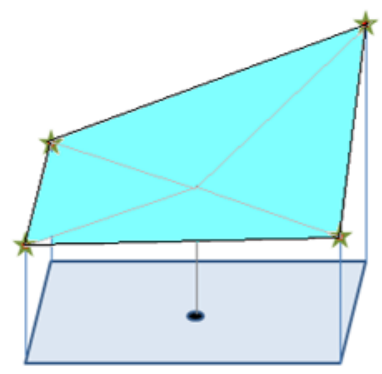

Figure 76: Average Plane Between Planar Edges

Each of these planes corresponds to a 2D flight condition data plane. The planes on the 2D plane are split up as shown in Figure 77: 

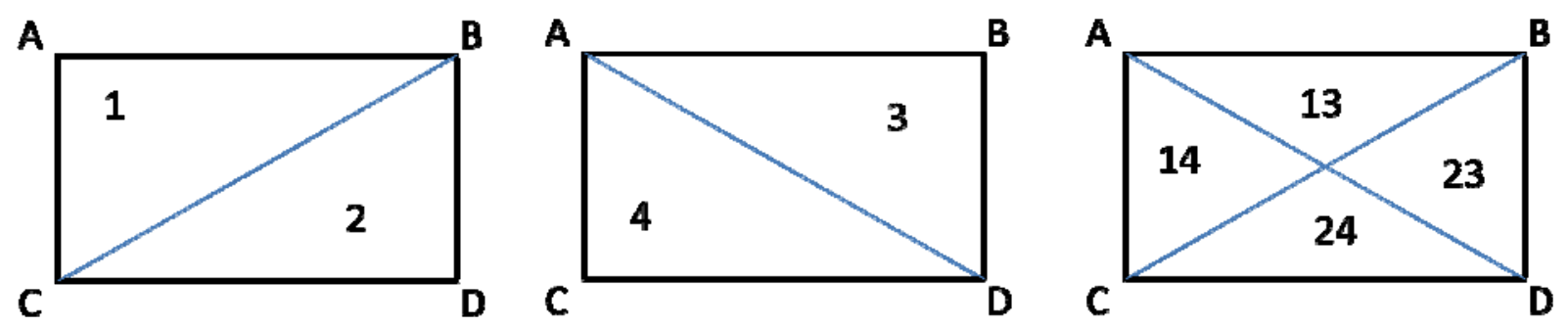

Figure 77: 2D Plane Assignment Breakdown

The location of intersection can be determined based on the flight parameter within the data grid. Logic is developed to limit the line for the altitude plane if the line is between points B and C. Similarly, the limit line for the mach plane is the line between points A and D. To establish this line, the fraction of altitude and mach is used to compare and find the max value for each other. This concept is shown in Figures 78 and 79.

A

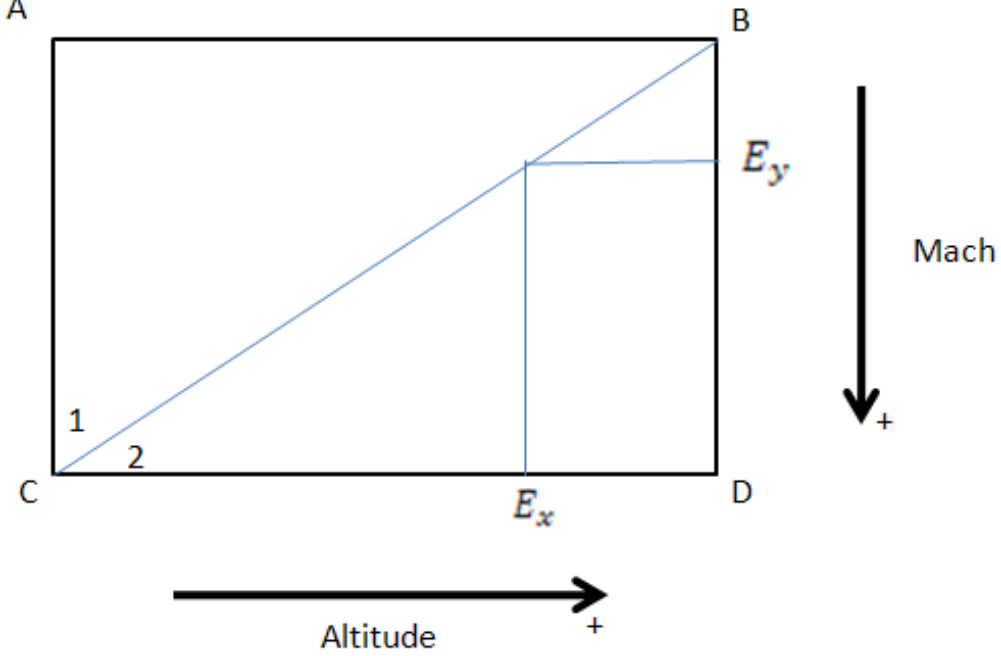

Figure 78: Determining limit line based on fraction of Mach and Altitude 


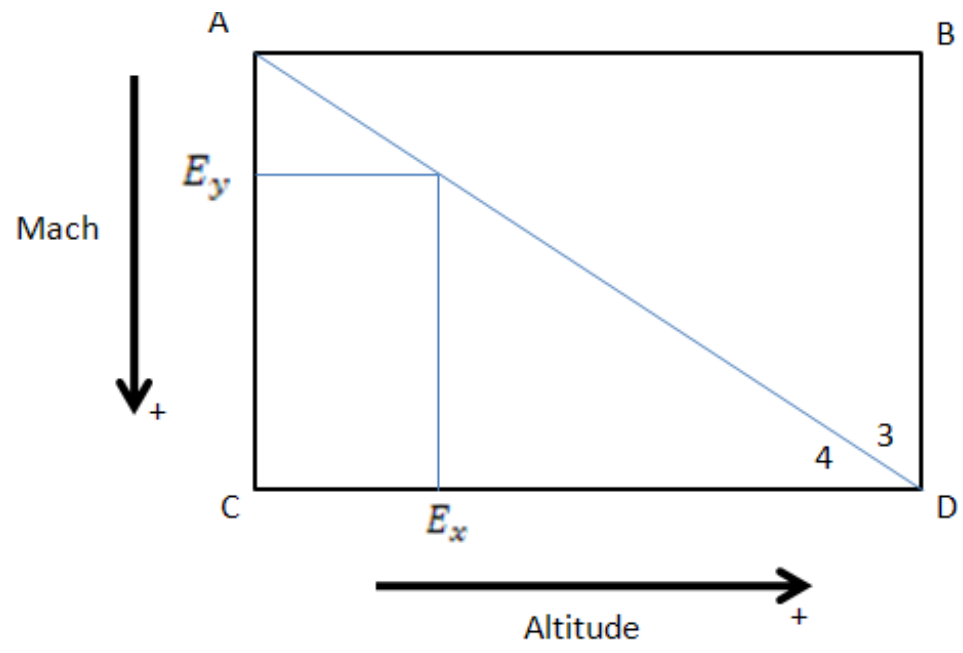

Figure 79: Determining limit line based on fraction of Mach and Altitude

\section{A.2.3 Derivation Equations-Planar Triangular Interpolation:}

Regular triangular interpolation techniques are performed for the relevant two planes of interest. The triangular interpolation process is shown through Figures 12-16 and its supporting charts and equations.

Note that: $A_{x}=C_{x}$ and $B_{x}=D_{x}$ and $A_{y}=B_{y}$ and $C_{y}=D_{y}$ where the $x$ subscript corresponds to altitude and the $y$ subscript corresponds to mach. $E_{x}, E_{y}$ respectively correspond to the flight altitude and mach. The $v$ subscripts correspond to the data value at the flight condition. $E_{v x}$ is the linearly interpolated value with respect to constant mach and $E_{v y}$ is the linearly interpolated value with respect to constant altitude. $E_{v x y}$ is the final triangular interpolated value. This information is visually shown in Figure 80 . 


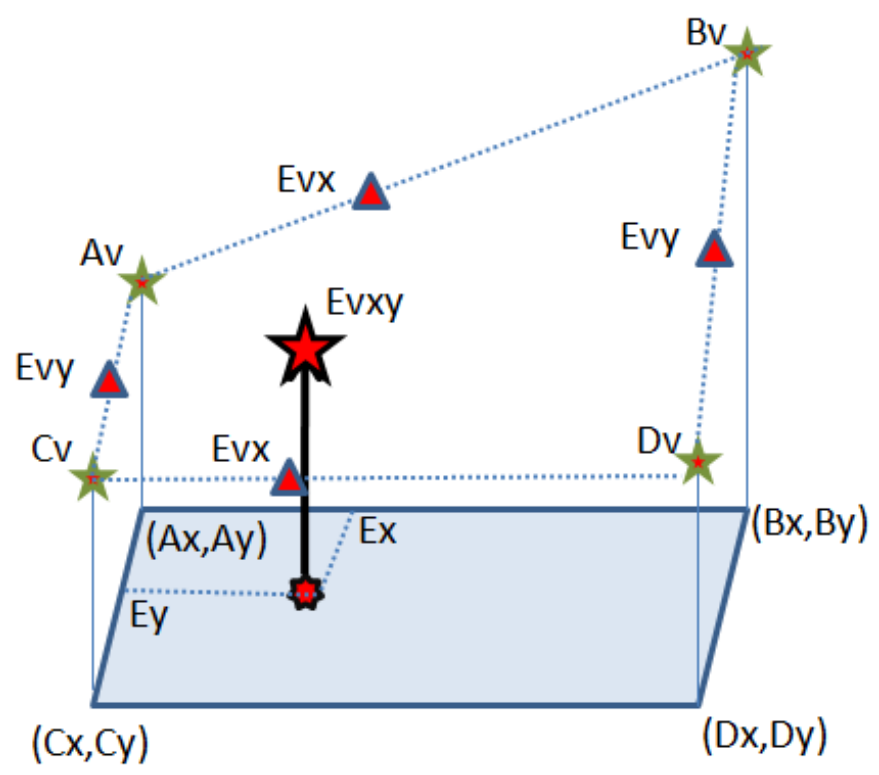

Figure 80: Interpolation Reference Values

\section{A.2.3.1 Plane 1:}

The values in the Figure 13 chart are used to perform the triangular interpolation.

\begin{tabular}{|c|c|c|c|}
\hline & $\mathrm{Ax}$ & $\mathrm{Ex}$ & $\mathrm{Bx}$ \\
\hline $\mathrm{Ay}$ & $\mathrm{Av}$ & Evx & $\mathrm{Bv}$ \\
\hline $\mathrm{Ey}$ & & Evxy & \\
\hline $\mathrm{Cy}$ & $\mathrm{Cv}$ & & $\mathrm{Dv}$ \\
\hline
\end{tabular}

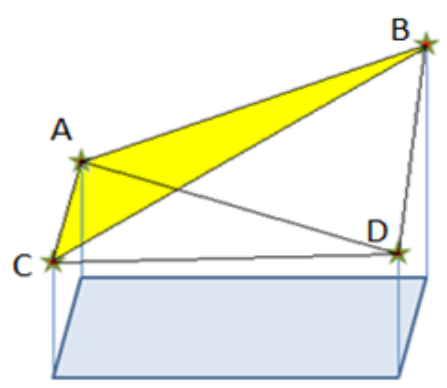

Figure 81: Plane 1 Reference Table and Schematic

First, interpolation is performed with respect to constant mach values.

$$
\begin{gathered}
\frac{E_{x}-A_{x}}{B_{x}-A_{x}}=\frac{E_{v x}-A_{v}}{B_{v}-A_{v}} \\
E_{v x}=A_{v}+\left(\frac{E_{x}-A_{x}}{B_{x}-A_{x}}\right)\left(B_{v}-A_{v}\right)
\end{gathered}
$$

Next, interpolation is performed with respect to constant altitude values. 


$$
\begin{gathered}
\frac{E_{y}-A_{y}}{C_{y}-A_{y}}=\frac{E_{v x y}-E_{v x}}{C_{v}-A_{v}} \\
E_{v, x y}=A_{v}+\left(\frac{E_{x}-A_{x}}{B_{x}-A_{x}}\right) *\left(B_{v}-A_{v}\right)+\left(\frac{E_{y}-A_{y}}{C_{y}-A_{y}}\right) *\left(C_{v}-A_{v}\right)
\end{gathered}
$$

The values in the Figure 14 chart are used to perform the triangular interpolation.

\begin{tabular}{|c|c|c|c|}
\hline & $\mathrm{Ax}$ & $\mathrm{Ex}$ & $\mathrm{Bx}$ \\
\hline $\mathrm{Ay}$ & $\mathrm{Av}$ & & $\mathrm{Bv}$ \\
\hline Ey & & Evxy & \\
\hline $\mathrm{Cy}$ & $\mathrm{Cv}$ & Evx & $\mathrm{Dv}$ \\
\hline
\end{tabular}

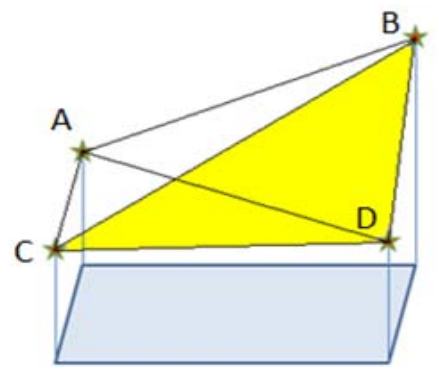

Figure 82: Plane 2 Reference Table and Schematic

First, interpolation is performed with respect to constant mach values.

$$
\begin{gathered}
\frac{B_{x}-E_{x}}{B_{x}-A_{x}}=\frac{D_{v}-E_{v x}}{D_{v}-C_{v}} \\
E_{v x}=D_{v}-\left(\frac{B_{x}-E_{x}}{B_{x}-A_{x}}\right)\left(D_{v}-C_{v}\right)
\end{gathered}
$$

Next, interpolation is performed with respect to constant altitude values.

$$
\begin{gathered}
\frac{C_{y}-E_{y}}{C_{y}-A_{y}}=\frac{E_{v x}-E_{v x y}}{D_{v}-B_{v}} \\
E_{v, x y}=D_{v}-\left(\frac{B_{x}-E_{x}}{B_{x}-A_{x}}\right)\left(D_{v}-C_{v}\right)-\left(\frac{C_{y}-E_{y}}{C_{y}-A_{y}}\right) *\left(D_{v}-B_{v}\right)
\end{gathered}
$$

The values in the Figure 15 chart are used to perform the triangular interpolation.

\begin{tabular}{|c|c|c|c|}
\hline & $\mathrm{Ax}$ & $\mathrm{Ex}$ & $\mathrm{Bx}$ \\
\hline $\mathrm{Ay}$ & $\mathrm{Av}$ & Evx & $\mathrm{Bv}$ \\
\hline Ey & & Evxy & \\
\hline $\mathrm{Cy}$ & $\mathrm{Cv}$ & & $\mathrm{Dv}$ \\
\hline
\end{tabular}

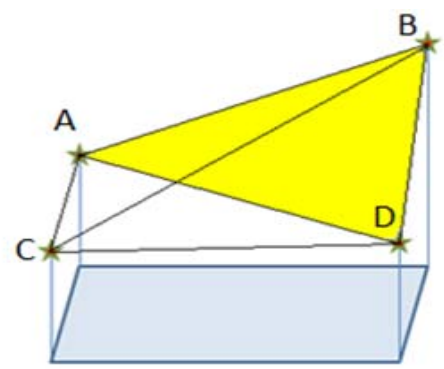

Figure 83. Plane 3 Reference Table and Schematic

First, interpolation is performed with respect to constant mach values. 


$$
\begin{gathered}
\frac{B_{x}-E_{x}}{B_{x}-A_{x}}=\frac{B_{v}-E_{v x}}{B_{v}-A_{v}} \\
E_{v x}=B_{v}-\left(\frac{B_{x}-E_{x}}{B_{x}-A_{x}}\right)\left(B_{v}-A_{v}\right)
\end{gathered}
$$

Next, interpolation is performed with respect to constant altitude values.

$$
\begin{gathered}
\frac{E_{y}-A_{y}}{C_{y}-B_{y}}=\frac{E_{v x y}-E_{v x}}{D_{v}-B_{v}} \\
E_{v, x y}=B_{v}-\left(\frac{B_{x}-E_{x}}{B_{x}-A_{x}}\right) *\left(B_{v}-A_{v}\right)+\left(\frac{E_{y}-B_{y}}{C_{y}-B_{y}}\right) *\left(D_{v}-B_{v}\right)
\end{gathered}
$$

The values in the Figure 16 chart are used to perform the triangular interpolation.

\begin{tabular}{|c|c|c|c|}
\hline & $\mathrm{Ax}$ & $\mathrm{Ex}$ & $\mathrm{Bx}$ \\
\hline $\mathrm{Ay}$ & $\mathrm{Av}$ & & $\mathrm{Bv}$ \\
\hline $\mathrm{Ey}$ & & Evxy & \\
\hline $\mathrm{Cy}$ & $\mathrm{Cv}$ & Evx & $\mathrm{Dv}$ \\
\hline
\end{tabular}

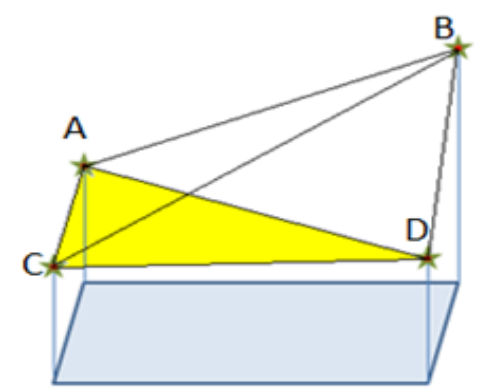

Figure 84. Plane 4 Reference Table and Schematic

First, interpolation is performed with respect to constant mach values.

$$
\begin{gathered}
\frac{E_{x}-A_{x}}{B_{x}-A_{x}}=\frac{E_{v x}-C_{v}}{D_{v}-C_{v}} \\
E_{v x}=C_{v}+\left(\frac{E_{x}-A_{x}}{B_{x}-A_{x}}\right)\left(D_{v}-C_{v}\right)
\end{gathered}
$$

Next, interpolation is performed with respect to constant altitude values.

$$
\begin{gathered}
\frac{C_{y}-E_{y}}{C_{y}-A_{y}}=\frac{E_{v x}-E_{v x y}}{C_{v}-A_{v}} \\
E_{v, x y}=C_{v}+\left(\frac{E_{x}-A_{x}}{B_{x}-A_{x}}\right)\left(D_{v}-C_{v}\right)-\left(\frac{C_{y}-E_{y}}{C_{y}-A_{y}}\right) *\left(C_{v}-A_{v}\right)
\end{gathered}
$$

\section{A.2.4 Derivation Equations-Fraction Determination:}

The altitude interval fraction is: 


$$
\text { Alt }_{\text {fraction }}=\frac{\left(E_{x}-A_{x}\right)}{\left(B_{x}-A_{x}\right)}
$$

Knowing that $B_{x}-A_{x}=5000 \mathrm{ft}$

Then

$$
\text { Alt }_{\text {fraction }}=\frac{\left(E_{x}-A_{x}\right)}{5000}
$$

And

$$
\left(1-\text { Alt }_{\text {fraction }}\right)=\frac{\left(B_{x}-E_{x}\right)}{5000}
$$

The mach interval fraction is

$$
\operatorname{Mach}_{\text {fraction }}=\frac{\left(E_{y}-A_{y}\right)}{\left(C_{y}-A_{y}\right)}
$$

Knowing that $C_{y}-A_{y}=0.05$ mach

Then

$$
\operatorname{Mach}_{\text {fraction }}=\frac{\left(E_{y}-A_{y}\right)}{0.05}
$$

And

$$
\left(1-\text { Mach }_{\text {fraction }}\right)=\frac{\left(C_{y}-E_{y}\right)}{0.05}
$$

Establish Mach and Altitude limits to create logic for determining which planes data points belong to:

$$
A l t_{\text {Limit }}=B_{x}-\left(\text { Mach }_{\text {fraction }} * 5000\right)
$$




$$
\text { Mach }_{\text {Limit }}=\left(\text { Alt }_{\text {fraction }} * 0.05\right)+A_{y}
$$

Then apply logic to determine which planes to reference:

If $A l t_{\text {Flight }} \leq A l t_{\text {Limit }}$ then go to plane 1, else go to plane 2

If Mach $_{\text {Flight }} \leq$ Mach Limit $_{\text {then }}$ go to plane 3, else go to plane 4

And the triangular interpolated values can be reduced to the following equations for each plane:

Plane 1:

$$
E_{v, x y}=A_{v}+\operatorname{Alt}_{\text {fraction }} *\left(B_{v}-A_{v}\right)+\operatorname{Mach}_{\text {fraction }} *\left(C_{v}-A_{v}\right)
$$

Plane 2:

$$
E_{v, x y}=D_{v}-\left(\left(1-\operatorname{Alt}_{\text {fraction }}\right) *\left(D_{v}-C_{v}\right)\right)-\left(\left(1-\text { Mach }_{\text {fraction }}\right) *\left(D_{v}-B_{v}\right)\right)
$$

Plane 3:

$$
E_{v, x y}=B_{v}-\left(\left(1-\operatorname{Alt}_{\text {fraction }}\right) *\left(B_{v}-A_{v}\right)\right)+\operatorname{Mach}_{\text {fraction }} *\left(D_{v}-B_{v}\right)
$$

Plane 4:

$$
E_{v, x y}=C_{v}+A l t_{\text {fraction }} *\left(D_{v}-C_{v}\right)+\left(\left(1-\text { Mach }_{\text {fraction }}\right) *\left(C_{v}-A_{v}\right)\right)
$$

Finally, the triangular interpolated point is at

$$
E_{V}=\frac{E_{v x y(\text { plane } 1 O R 2)}+E_{v x y(\text { plane } 3 O R 4)}}{2}
$$

The DLTI method is analogous to creating a polygon mesh for a surface interpolation where the interpolation space is characterized by two-dimensional piecewise functions. In theory, this technique can be repeated with higher precision breaking down each rectangle grid into four additional sub-rectangles using the average point as a new reference edge point. This 
process will begin to emulate the results for a traditional bilinear interpolation but will require more calculations. For applications where strict linearity is required, the DLTI can be applied, where greater precision can be balanced against available computation time. For interpolation in the F/A-18 simulator, only one iteration of the DLTI is required.

Figure 85 shows a visual comparison of localized interpolation results from triangular interpolation (based on only three points), bilinear interpolation, and double linear triangulation interpolation.

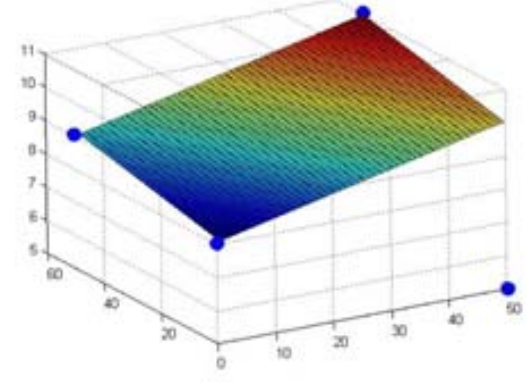

(a)

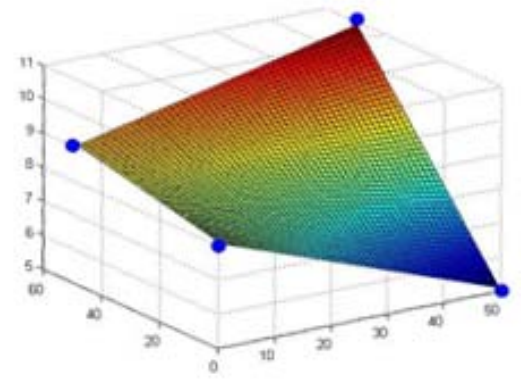

(b)

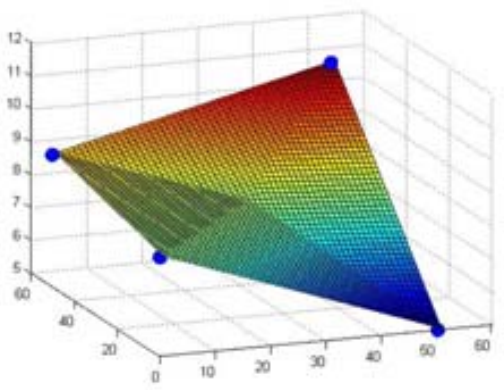

(c)

Figure 85: a) Triangular Interpolation (b) Bilinear Interpolation (c) DLTI Again, triangular interpolation does not incorporate all four reference points. Bilinear interpolation is actually a quadratic interpolation scheme. DLTI mimics a bilinear interpolation, but remains a linear process. Figure 18 shows a visual representation of applying the double linear triangular interpolation across the entire expected F/A-18 simulation flight envelope on a unit-less single matrix element of the ASE models. 
Figure 86 shows a visual representation of the double linear triangular interpolation results for a single matrix element throughout the entire flight envelope.

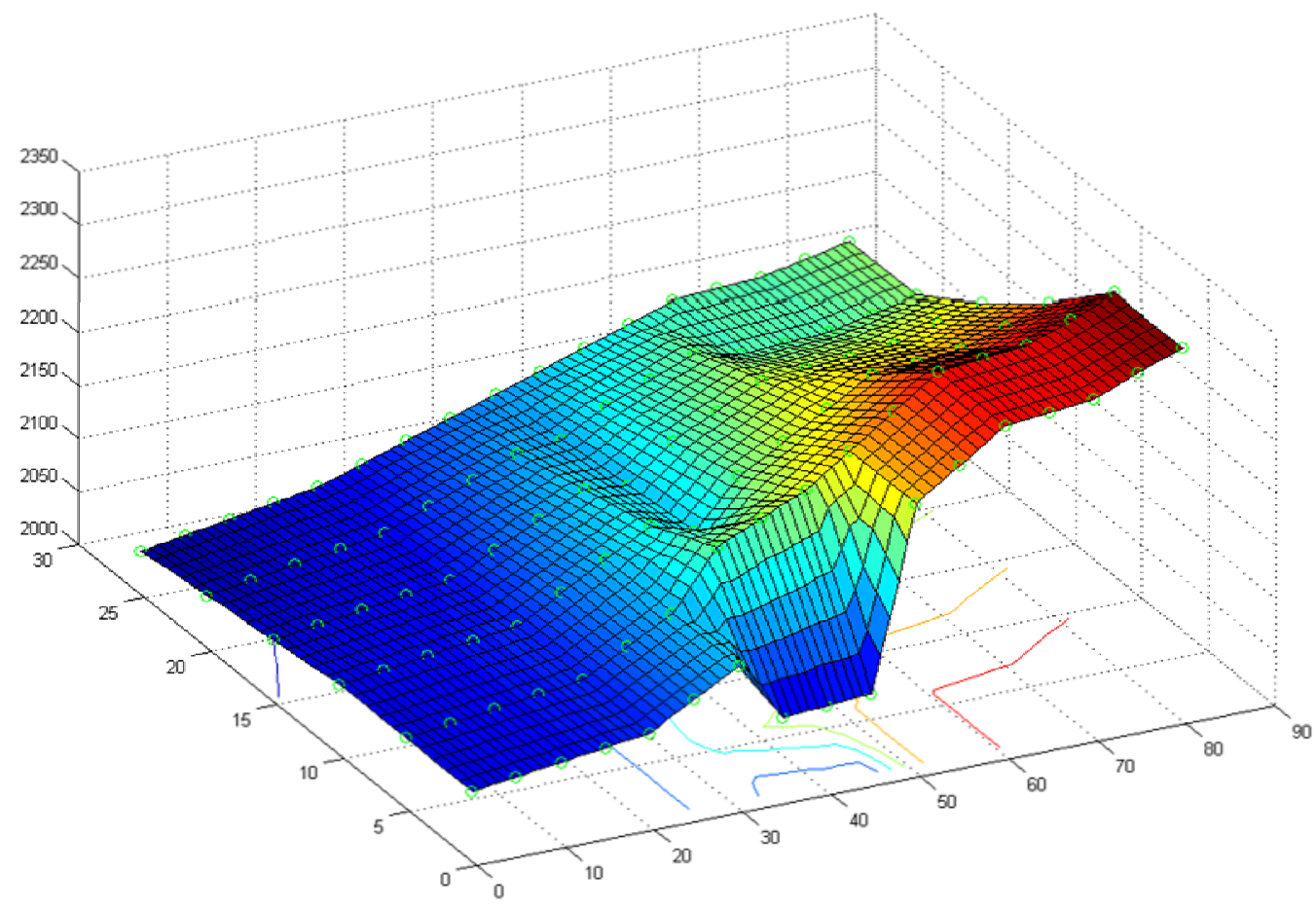

Figure 86: Example Matrix Element Using Double Linear Interpolation Scheme Through All Flight Conditions 


\title{
Appendix B: Code Repository and Descriptions
}

\author{
B.1 Matlab Code
}

1. $\mathrm{ABCDX}$

a. STEP_1_\&_3_Master_Data_Combiner.m

i. Takes raw aaw data in '.mat' format and transfers into workspace in workable matrix breakdown form (A_050_05...)

1. Make sure file directory location of files is correct

b. STEP_2A_Data_Reference_Filler.m

i. Linear Interpolation (based on table, see hard copy)

c. STEP_2B_Data_Reference_Filler_Duplicate.m

i. For copying data points going across constant mach values at a constant altitude(ex. Alt=5-10k, Mach=0.50)

d. STEP_4_Three_Dimensional_Matrix_Combiner.m

i. Combines all data into master baseline matrix

e. STEP_4B_Three_Dimensional_Matrix_Comb_Discr.m

i. Also discretizes data, for direct transfer to FORTRAN

2. PLANTX

a. Same process as above, except for using 'plantx'

b. STEP_2B_data_reference_filler_constant.m

i. Duplicating with respect to constant mach

c. STEP_2B_data_reference_filler_constant_alt.m 
i. Duplicating with respect to constant altitude

3. For post data creation, code created and converted to FORTRAN use.

a. master_data_combiner3_STEP_1.m

i. Takes raw aaw data in '.mat' format and transfers into workspace in workable matrix breakdown form (A_050_05...)

b. ETC. $\wedge$

c. MEGA_Discretizer.m

i. Discretize the data

d. DAT_file_writer.m

i. Save master matrices as '.dat' files

e. Y_out_and_surf_plot.m

i. Plot y-outputs for a given u-input condition for all flight conditions

f. Data_trend_plotter_baseline.m

i. Create 3D plot of baseline data of specified element of matrix

g. MEGA_Matrix_Creator_FULL_rev_8_9_10.m

i. Used to take baseline matrix and use double linear triangular interpolation to fill inbetween data points

h. Data_trend_plotter.m

i. General plotter to plot master matrice

4. Analyzing Data:

a. MEGA_Matrix_Creator_FULL_rev_8_10.m

i. (Assumes workspace is saved where 3D master baseline matrices have already been created) 
ii. Takes baseline data and performs double triangular linear interpolation across all intermediate flight conditions, spaced out as: Alt $=[5000: 1000: 30000]$ and Mach $=[0.50: 0.01: 1.30]$ for a total depth of $2106(26 * 81)$

iii. Also discretizes data

b. data_trend_plotter_all.m

i. Reads in Master matrices and plots a single elements from the matrices for all flight conditions

ii. Also reads in baseline data points and scales with master matrice data

iii. Creates 'surf' plot of master data, and adds baseline data points as dots

iv. Can change element location to see varying surf plot changes throughout the elements of the matrices of interest

c. Variable_interp_THROUGH_TIME.m

i. Creates steady state time plot of ASE model (verifies stability). Can change input flight conditions for reference

d. Variable_interpolator_7_master_ref_disc.m

i. Performs entire 


\section{B.2 FORTRAN Code}

1. ASE_interp_init.F

a. Initialize loading of data

2. ASE_interp_exec.F

a. Choice of abcdx or plantx operations

3. loads.def

a. Description of variables used

4. loads.cmn

a. Define new variables used

5. Sens_in.F

a. Point of entry/addition of ASE components into the F18 Sim

The F18 simulator is made up of multiple source code files, with many of the subroutines written and compiled in FORTRAN. Completely independent subroutines were written for integrating ASE components into the sim.

The ASE_interp_init.F file loads in the data matrices for the ABCDX and PLANTX matrices. This is only called once when the sim is activated.

ASE_interp_exec.F is the full processing algorithm that reads in flight conditions (altitude and mach), and actuator commands or positions. The flight conditions dictate which reference points are pulled up and undergoes an interpolation scheme to match the flight condition of interest.

loads.def defines all of the new variable names that are used

loads.cmn defines the name and dimensional sizes of the variables 
Sens_in.F Pulls in rigid body and ASE data results. Creates logic to turn ASE on or off based on commands and calculates percentage impact of ASE components compared to rigid body components. Figures $85-87$ provide visualizations of where the aforementioned code is placed into the F/A-18 simulator.

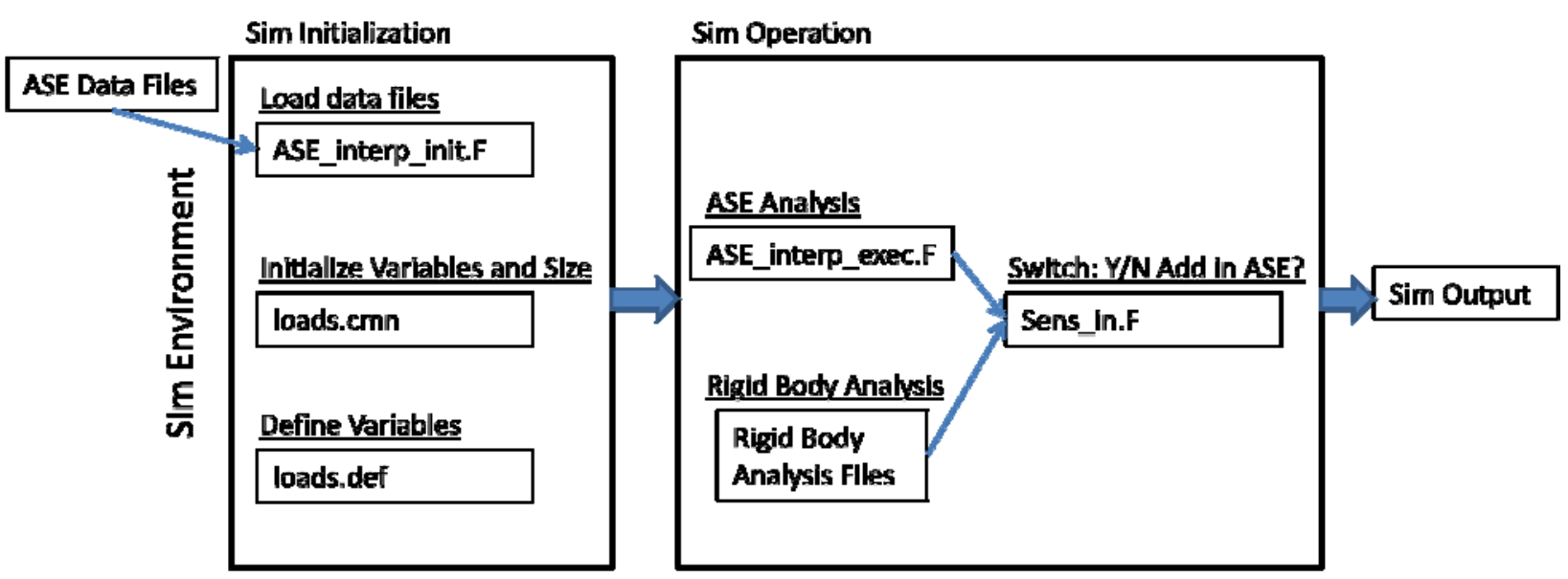

Figure 87: computation process in F18 Sim (Flow diagram of processes)

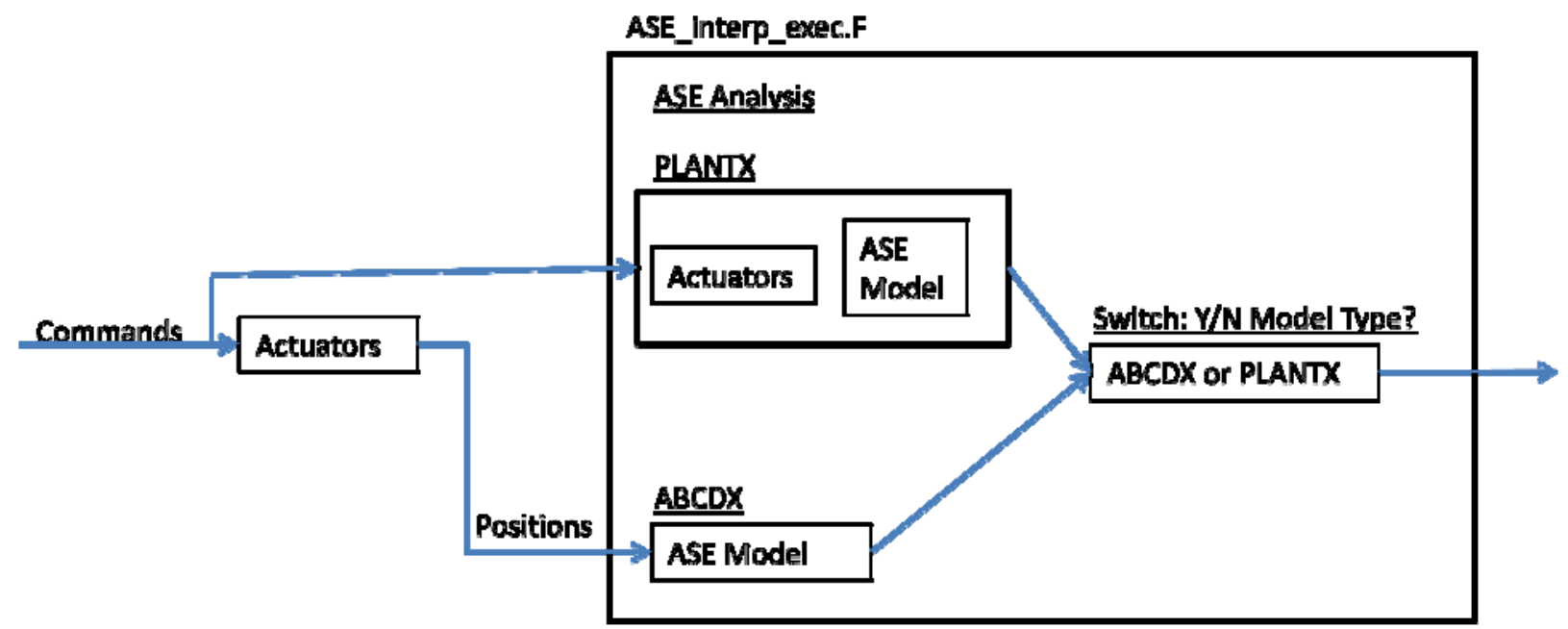

Figure 88: Specific IF logic for ASE 


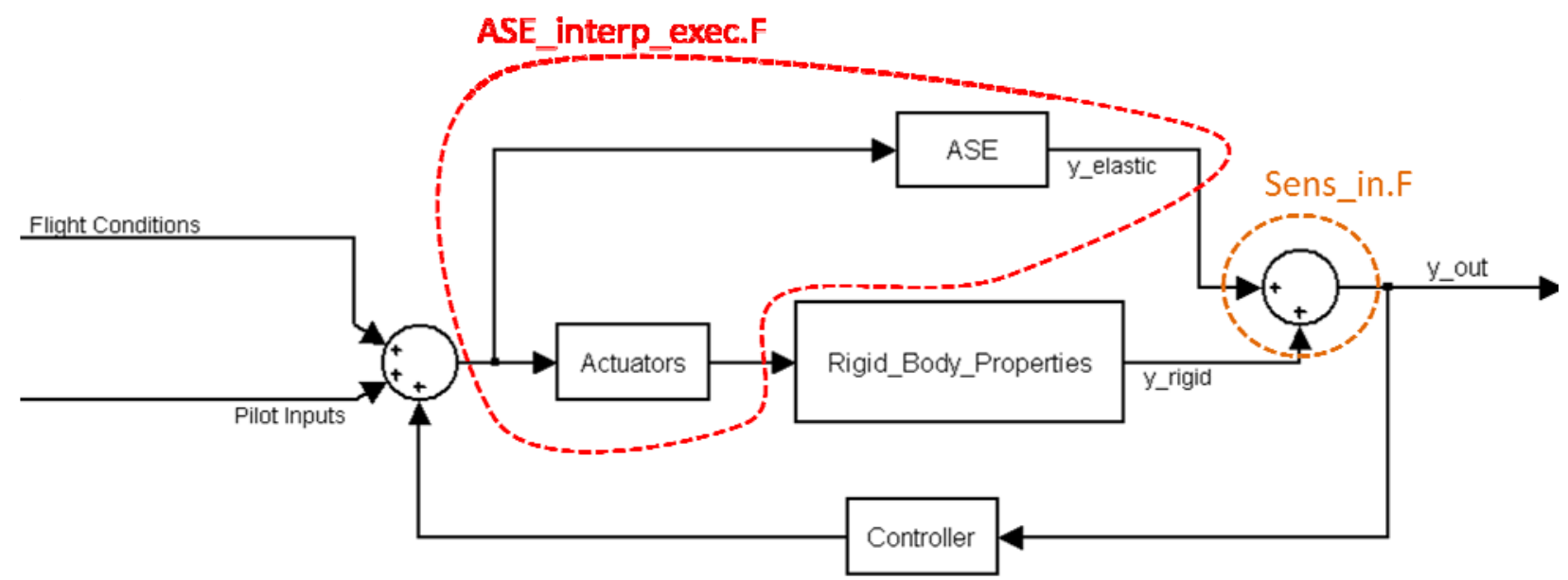

Figure 89: Flow Diagram Highlighting Location of FORTRAN Files for Sim 